\title{
NANOANALYSE HÖCHSTER AUfLÖSUNG VON METALLISCHEN SCHICHTSYSTEMEN
}

Dissertation zur Erlangung des Doktorgrades der Mathematisch-Naturwissenschaftlichen Fakultäten der Georg-August-Universität zu Göttingen

vorgelegt von

Jörg Schleiwies aus Lingen 
D7

Referent: Prof. Dr. R. Kirchheim

Korreferent: Prof. Dr. W. Schröter

Tag der mündlichen Prüfung: 03.05.2001 
1 EINLEITUNG 1

2 GRUNDLAGEN DER INTERREAKTION 4

2.1 Empirische Regeln zur Phasenbildung 4

2.2 Phasenwachstum und -selektion nach dem Modell von
GÖSELE und TU

2.2.1 Phasenwachstum 6

2.2.2 Phasenselektion 9

2.3 Keimbildungs- und Wachstumsmodell nach COFFEY 10

2.4 Einfluß der Mikrostruktur 11

$3 \quad$ EXPERIMENTELLE METHODEN 13

3.1 Feldionenmikroskop (FIM) und Tomographische Atomsonde (TAP) 13

$\begin{array}{lll}3.1 .1 & \text { Das Feldionenmikroskop } & 13\end{array}$

3.1.2 Die Tomographische Atomsonde (TAP) 15

3.1.3 Datenanalyse 18

3.2 Herstellung von TAP-Proben durch Ionenstrahlsputterdeposition 20

3.3 Schichtherstellung der planaren $[\mathrm{Co} / \mathrm{Cu} / \mathrm{Py} / \mathrm{Cu}]_{\mathrm{N}}-$ Systeme 21

3.4 Lithographische Präparation planarer Schichten für die

$\begin{array}{ll}\text { Tomographische Atomsonde } & 22\end{array}$

3.5 Präparation von Spitzen mit dem SekundärIonen-

3.6 Elektronenmikroskopie 25

$\begin{array}{lll}3.7 & \text { Röntgendiffraktometrie } & 27\end{array}$

$\begin{array}{lll}3.8 & \text { Magnetowiderstandsmessungen } & 27\end{array}$

4 NANOANALYSE DER INTERREAKTION VON

AG/AL-SCHICHTPAKETEN 29

4.1 Vorbemerkungen 29

4.2 Experimentelle Vorarbeiten 31 
4.3 Ergebnisse mit der Tomographischen Atomsonde 33

4.3.1 Eigenschaften der gesputterten Schicht 33

4.3.2 Experimentelle Ergebnisse der Tomographischen Atomsonde 36

4.4 Diskussion der Ergebnisse $\quad 47$

4.4.1 Diffusionsverhalten des planaren Reaktionsstadiums $\quad 47$

4.4.2 Struktur der intermediären Phase $\mathrm{Al}_{2} \mathrm{Ag} \quad 49$

4.4.3 Thermodynamische Eigenschaften des Systems 52

4.4.4 Kinetische Stabilisierung der Vorläuferphase 55

4.4.5 Modellvorstellung des Reaktionsmechanismus $\quad 57$

4.5 Zusammenfassung der Ergebnisse zum System Ag/Al 59

5 NANOANALYTIK VON CO/CU/PY-SCHICHTSYSTEMEN 61

5.1 Vorbemerkungen 61

5.2 Experimentelle Vorarbeiten 64

5.3 Charakterisierung mit Hilfe der Tomographischen Atomsonde 68

5.3.1 Herstellung der Proben 68

5.3.2 Experimentelle Ergebnisse der Tomographischen Atomsonde 71

5.4 Diskussion der Ergebnisse $\quad 80$

5.5 Zusammenfassung der Analyse von $\mathrm{Co} / \mathrm{Cu} / \mathrm{Py}-$ Schichten 83

6 ABSCHLUSSBEMERKUNG 85

$\begin{array}{lr}\text { ANHANG } & 86\end{array}$

$\begin{array}{lr}\text { ABKÜRZUNGSVERZEICHNIS } & 88\end{array}$

$\begin{array}{lr}\text { LITERATURVERZEICHNIS } & 89\end{array}$ 


\section{Einleitung}

In den letzten Jahren hat das Gebiet der Festkörperreaktion im Bereich der Dünnfilmanwendung stetig wachsendes Interesse auf sich gezogen. Heutige technologische Anwendungen profitieren von Schichtstrukturen auf kleinster Skala. Sie finden in der Mikroelektronik als Leiterbahnen oder Transistoren in hochintegrierten Bauteilen Verwendung oder werden als Diffusions- und Reaktionsbarrieren eingesetzt. Insbesondere die Kombination magnetischer und nicht-magnetischer Komponenten unter Ausnutzung des GMR-Effektes (GiantMagnetoResistance) ist sowohl für die Datenverarbeitung (MRAM und Festplattenleseköpfe) als auch für die Automobil- (z.B. als Drehzahlmesser beim ABS) und die Elektronikindustrie (z.B. als winkelauflösende Detektoren für berührungslose Einstellung von Waschprogrammen) von großem Interesse. Dabei setzt allerdings auch die gezielte Modifizierung und auf eine bestimmte Anwendung bezogene Optimierung dieser Systeme die Kenntnis ihrer Reaktionseigenschaften voraus.

$\mathrm{Da}$ die Sensoren in der industriellen Anwendung Schichtdicken von wenigen Nanometern besitzen, ist die Temperaturstabilität der Systeme von großer Bedeutung. Befinden sich zwei mischende Komponenten im Kontakt, werden sich im Verlauf der Festkörper-Interreaktion Phasen bilden, die auf die Eigenschaften des Systems, wie magnetische, elektronische und mechanische, erheblichen Einfluß haben. Die Phasenbildung findet an der Grenzfläche jedoch nicht für alle im Phasendiagramm vorkommenden Phasen gleichzeitig statt, sondern in einer für das System eigenen Reihenfolge. Es existieren eine Reihe von empirischen Regeln, die Aussagen über eine Phasenselektion oder -wachstum treffen. GöSELE und TU stellen in ihrem Modell kinetische Überlegungen in den Vordergrund [GöselE1, GöselE2]. Aufgrund der Wachstumsgeschwindigkeit der einzelnen Phasen wird auf deren Stabilität und die Reihenfolge der Phasenbildung geschlossen. Die im Modell verwendeten kinetischen Parameter, insbesondere etwa Grenzflächenreaktionskonstanten, sind experimentell nur schwer zugänglich, so daß Aussagen über reale Schichtsysteme kaum möglich sind. Zusätzlich wird eine planare Geometrie der Produktphasen vorausgesetzt, die nicht immer gegeben ist.

Experimentell zugänglicher sind Bildungsenthalpien, die in der empirischen Regel von Pretorius verwendet werden [Pretorius1, Pretorius2]. Neben kinetischen Betrachtungen werden dadurch auch thermodynamische Aspekte berücksichtigt. Gemäß PRETORIUS wird zuerst die Phase gebildet, die die höchste effektive Bildungsenthalpie bei der Zusammensetzung der niedrigsten Liquidustemperatur aufweist. Für eine Vielzahl von Systemen liefert diese Regel eine richtige Vorhersage.

Als erster nimmt COFFEY in seinem zweistufigen Reaktionsmodell Abstand von einer vorausgesetzten Lagenstruktur [COFFEY]. Die aus dem Modell berechneten Keimgrößen erscheinen mit mehreren $10 \mathrm{~nm}$ jedoch extrem hoch. Auch dieses Modell berücksichtigt die vorhandene Mikrostruktur und ihren Einfluß auf die Keimbildung in ungenügender Weise. Insbesondere werden signifikant 
unterschiedliche Diffusionskoeffizienten ebenso vernachlässigt, wie der Einfluß der Korngrenzendiffusion.

Ein wesentlicher Grund für die nur eingeschränkte Gültigkeit der Modelle ist bei den oft unzureichenden experimentellen Daten zu suchen. Weit verbreitete Methoden wie RBS oder SIMS ermöglichen auf lateraler Skala nur unzureichende Auflösung. Für die chemische Charakterisierung der Schichtreaktion auf derart niedriger Längenskala existieren nur wenige hochentwickelte Methoden. Die hochauflösende Transmissionselektronenmikroskopie ermöglicht eine hohe laterale Auflösung, in die Tiefe ergibt sich jedoch ein Summationseffekt über die Schichtdicke. Daher ist eine chemisch scharfe, aber geometrisch unebene Grenzfläche nicht von einer geometrisch ebenen, aber chemisch unscharfen Grenzfläche zu unterscheiden. Die in dieser Arbeit schwerpunktmäßig benutzte Tomographische Atomsonde ist hingegen in der Lage, auch komplizierte 3D-Reaktionsmorphologien atomar genau zu analysieren. Das ausgewählte Volumen liegt in der Regel bei $15 \cdot 15 \cdot 100 \mathrm{~nm}^{3}$ und sollte ausreichend sein, um Antwort über Diffusionswege und Eigenschaften von Grenzflächen als Keimbildungszentren zu geben.

Der Einfluß der Mikrostruktur auf den Reaktionsmechanismus dünner Schichten soll in der vorliegenden Arbeit anhand von zwei Beispielsystemen näher betrachtet werden.

In Kapitel 4 wird anhand des scheinbar klaren Modellsystems Ag/Al die Bedeutung der Mikrostruktur für die Keimbildung und Wachstum der Produktphase demonstriert. Die Berichte der einschlägigen Literatur lassen bei einer Wärmebehandlung nur die Bildung einer Produktphase erwarten. DSC-Messungen von RoY zeigen jedoch das Auftreten von zwei exothermen Wärmetönungen [RoY]. Eine Interpretation nach COFFEY als zweistufiger Prozess erklärt zwar das Auftreten des Doppelpeaks, liefert für die Keimgröße aber unvernünftig hohe Werte. Die an diesem Punkt ansetzende Analyse der Tomographischen Atomsonde mit ihrer hohen chemischen Auflösung enthüllt eine komplizierte dreidimensionale Reaktionsmorphologie.

Ein weiteres Beispiel dafür, daß selbst nach Vorliegen einer präparierten Lagenstruktur die Reaktionsmechanismen keineswegs vorgegeben sind, ist in Kapitel 5 gegeben. Anhand der Nanoanalyse des technologisch aktuellen $\mathrm{Co} / \mathrm{Cu} / \mathrm{Fe}_{19} \mathrm{Ni}_{81}{ }^{1}$-Schichtsystems, werden Rückschlüsse auf mögliche Versagensmechanismen des Magnetowiderstandes gezogen und mit Vorschlägen aus der Literatur verglichen. Nur mit detailgenauer Kenntnis der inneren Abhängigkeiten ist eine zielgerichtete Optimierung dieser Systeme möglich.

Zunächst soll aber in Kapitel 2 eine kurze Einführung in die aktuellen Fragen und vorhandenen Modelle der Interreaktion gegeben, um eine gemeinsame Basis für das Verständnis $\mathrm{zu}$ schaffen. Kapitel 3 beschäftigt sich mit den experimentellen Methoden, die zur Analyse verwandt wurden. Das Hauptaugenmerk liegt dabei auf

\footnotetext{
${ }^{1}$ Für die Legierung $\mathrm{Fe}_{19} \mathrm{Ni}_{81}$ wird auch das Synonym Permalloy benutzt.
} 
der Tomographischen Atomsonde, deren Ergebnisse den Schwerpunkt der Arbeit bilden. Die dafür aufwendige Schichtpräparation erfolgte in einer im Rahmen dieser Arbeit aufgebauten Sputteranlage, die ebenfalls näher beschrieben wird. Sie ermöglicht die notwendige in-situ Ionenstrahlreinigung der Substratoberfläche direkt vor der Schichtdeposition ohne Bruch des Vakuums zur Verbesserung der mechanischen Stabilität der Schichten beim Analyseprozeß.

Neben der direkten Beschichtung von Proben in der für die TAP-Untersuchungen typischen Spitzengeometrie wird eine neuartige lithographische Präparationsmethode erfolgreich demonstriert, die es ermöglicht, aus planaren Schichtsystemen Proben für die TAP-Analyse mit einem Krümmungsradius der Spitze kleiner $50 \mathrm{~nm} \mathrm{zu}$ präparieren. 


\section{Grundlagen der Interreaktion}

Die Frühstadien der Interreaktion verschiedener Materialien sind ein hochaktuelles Forschungsgebiet. Betrachtet man ein mischendes binäres System mit mehreren auftretenden Phasen, so wird in den Frühstadien an der Grenzfläche nur die Bildung einer Phase beobachtet. Zu späteren Zeiten sind dann alle möglichen Phasen in einer Schichtfolge im System zu finden. In der Literatur werden verschiedene empirische Modelle zur Erklärung dieser Phasenauswahl diskutiert. Sie treffen für eine Vielzahl von Systemen zu. Ein grundsätzliches physikalisches Verständnis fehlt jedoch bis heute.

\subsection{Empirische Regeln zur Phasenbildung}

Um die Frage zu beantworten, welche Phase zuerst gebildet wird, wurden eine Reihe von einfachen Regeln entwickelt, die sich auf die Thermodynamik und das Phasendiagramm des Systems stützen.

Die Regel von WALSER und BENÉ stützt sich dabei auf die reine Betrachtung des Atomtransportes im Volumen. Demnach bildet sich diejenige kongruent schmelzende Gleichgewichtsphase zuerst, die am nächsten zum tiefsten Eutektikum des Gleichgewichtsphasendiagramms liegt [WALSER]. Die Regel begründet sich in der Annahme, daß an der Grenzfläche immer eine Durchmischungszone mit der Zusammensetzung des tiefsten Eutektikum gebildet wird, da so eine größtmögliche Mobilität und Durchmischung vom System erreicht werden kann. In ähnlicher Form schlägt D’HEURLE vor, daß sich zuerst die Phase mit der höchsten Diffusivität bildet, was sich oft auch als richtig Vorhersage erweist [HEURLE1].

Eine weitere, bei vielen Systemen sehr erfolgreiche Regel, stammt von PRETORIUS. Er erweitert die rein kinetischen Argumente von WALSER und BENÉ um eine thermodynamische Komponente [Pretorius1, Pretorius2]. Dazu führt er das Konzept der effektiven Bildungsenthalpie ein. Ausgangspunkt ist die Änderung der Freien Enthalpie $\Delta \mathrm{G}^{0}$ :

$$
\Delta G^{0}=\Delta H^{0}-T \Delta S^{0},
$$

mit der Annahme, daß der entropische Term vernachlässigt werden darf. Im folgenden wird deshalb nur die Enthalpie $\Delta \mathrm{H}^{0}$ betrachtet, die experimentell leichter zugänglich ist und von PRETORIUS als treibende Kraft für die Phasenbildung verantwortlich gemacht wird. Ähnlich wie WALSER und BENÉ nimmt er an, daß zu Anfang der Reaktion eine Mischzone gebildet wird, die der Zusammensetzung $c_{e}$ des tiefsten Schmelzpunktes des Systems entspricht, da hier die größte Mobilität der Komponenten vorliegt. In dieser Zone kommt es dann zur Keimbildung der Phase (Konzentration $c_{\text {phase }}$ ) mit der höchsten treibenden Kraft. Zur Einbeziehung der Tatsache, daß die Ausgangszusammensetzung nicht immer der Stöchiometrie der gebildeten Phase entspricht, benutzt man zur Beschreibung der treibenden Kraft die effektive Bildungsenthalpie $\Delta \mathrm{H}_{\text {eff: }}$ : 


$$
\Delta H_{\text {eff }}=\frac{c_{e}}{c_{\text {phase }}} \cdot \Delta H^{0} .
$$

Sind die Bildungsenthalpien der Phasen eines Systems bekannt, so läßt sich ein Diagramm wie in Teilbild b) der Abbildung 2-1 erstellen. Dazu trägt man die ermittelten Bildungsenthalpien über der jeweiligen Phasenkonzentration auf und verbindet die Punkte zu den Enden der Reinkomponenten. Da das System eine maximale Erniedrigung der Freien Energie anstrebt, wird die Phase mit der negativsten effektiven Bildungsenthalpie zuerst auftreten.

Als ein eindrucksvolles Beispiel der richtig vorhergesagten Phasenauswahl ist das System $\mathrm{Al}-\mathrm{Au} \mathrm{zu}$ sehen. In Abbildung 2-1 sind das Phasendiagramm und die effektiven Bildungsenthalpien nach PRETORIUS dargestellt. Der tiefste Schmelzpunkt des Systems liegt am Eutektikum mit 78 at.-\% Au.

b)

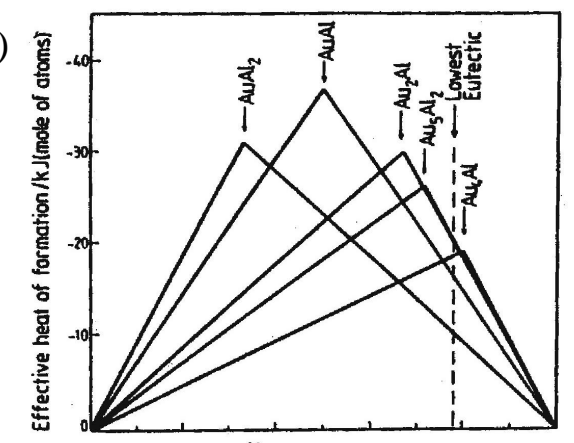

a)

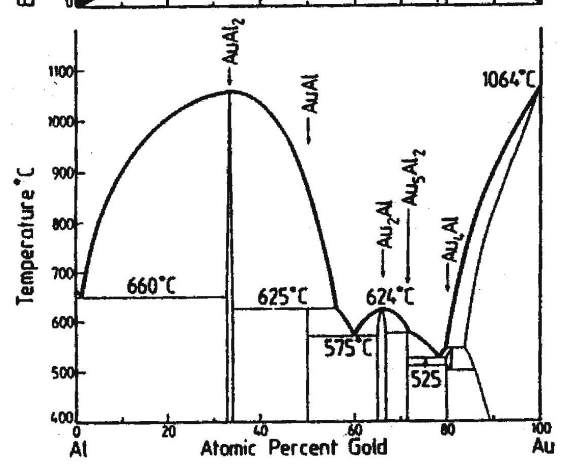

Abbildung 2-1: a) Phasendiagramm Al-Au. b) Effektive Bildungsenthalpien nach [PRETORIUS1]

An dieser Stelle des Phasendiagramms haben zwei Phasen (abzulesen in Teilbild b) eine sehr ähnliche effektive Bildungsenthalpie, die deutlich über der der verbleibenden Phase liegt, $\mathrm{Au}_{2} \mathrm{Al}$ und $\mathrm{Au}_{5} \mathrm{Al}_{2}$. Wegen der geringen Differenz werden je nach Anfangsbedingungen der Experimente, beide als erste gebildete Phase berichtet [MAJnI, CAMPISANO]. Aus rein thermodynamischer Sicht müßte sich AuAl als stabilste Phase zuerst bilden. Wohingegen die Regel von WALSER und BENÉ unter ausschließlicher Berücksichtigung kinetischer Gesichtspunkte die Bildung von $\mathrm{Au}_{4} \mathrm{Al}$ vorhersagt. Nur die Kombination aus Kinetik und Thermodynamik gemäß PRETORIUS erlaubt die richtige Vorhersage der Phasenbildung. 
Experimentell zeigt sich sehr oft die Bildung einer metastabilen Vorläuferphase vor der Keimbildung der eigentlich erwarteten Gleichgewichtsphase [GÄRTNER]. Das Auftreten eines metastabilen kristallinen oder amorphen Vorläufers kann weder mit der empirischen Regel von WALSER und BENÉ noch nach PRETORIUS erklärt oder vorhergesagt werden.

\subsection{Phasenwachstum und -selektion nach dem Modell von GöSELE und TU}

Das im folgenden vorgestellte Modell von GöSELE und TU [GöSELE1, GöSELE2] berücksichtigt keine spezifische Thermodynamik des Systems, betrachtet aber neben der Volumendiffusion auch den Transport über die Grenzflächen und ermöglicht deshalb sowohl die Beschreibung von Phasenwachstum und -selektion als auch des Auftretens von metastabilen Vorläuferphasen.

\subsubsection{Phasenwachstum}

Voraussetzung für die folgende Betrachtung ist bereits die Existenz einer Reaktionsphase. Weiterhin werden Grenzflächenreaktionsbarrieren berücksichtigt, die die Einstellung der Gleichgewichtskonzentration an den Grenzflächen behindern und so im Gegensatz zum volumenkontrollierten Wachstum zu endlichen Reaktionsraten am Anfang der Reaktion führen. Wäre das Wachstum der Produktphasen von Anfang an volumenkontrolliert (parabolisches Wachstum), so wäre die Koexistenz aller Phasen zu jeder Zeit möglich und eine Selektion aus kinetischen Gründen nicht zu erklären.

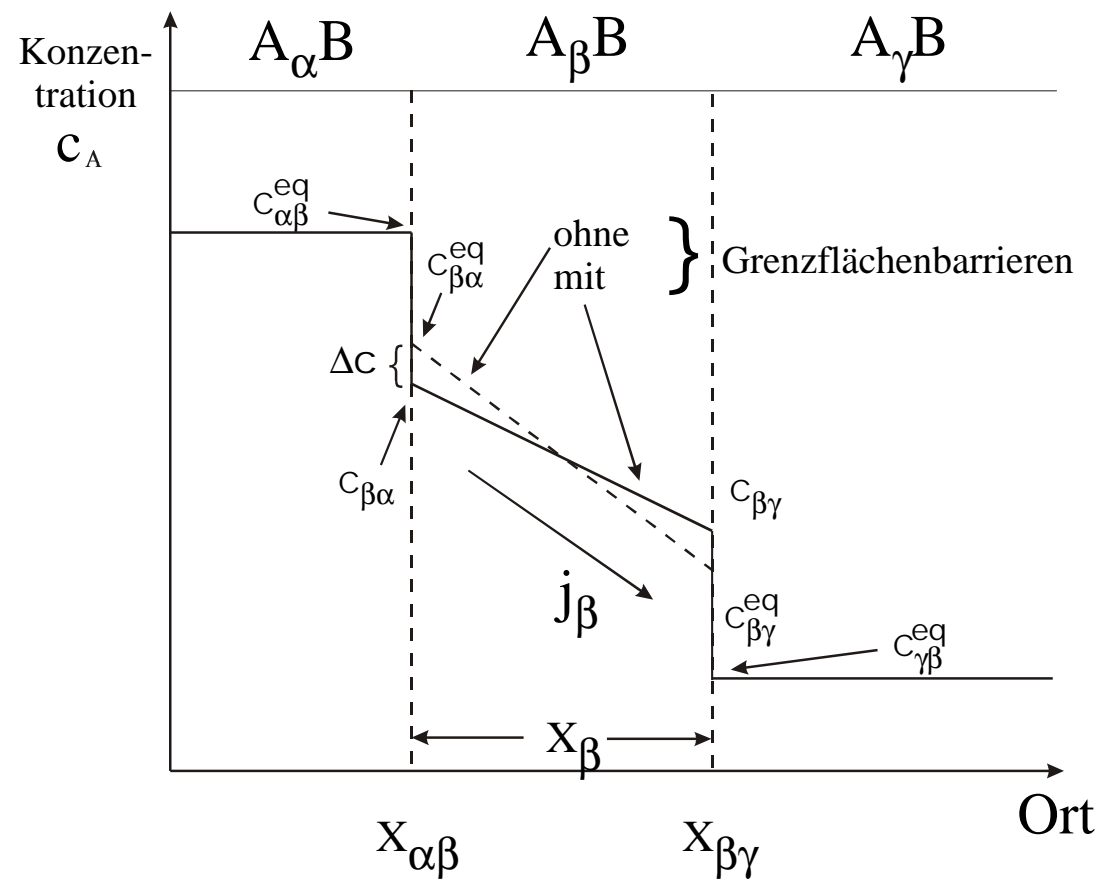

Abbildung 2-2: Konzentrationsverlauf in einem Reaktionspaar A und B mit und ohne Grenzflächenreaktionsbarrieren. 
In Abbildung 2-2 ist der Konzentrationsverlauf eines Reaktionspaares $\mathrm{AB}$ mit einer intermetallischen Phase $\mathrm{A}_{\beta} \mathrm{B}$ mit und ohne Grenzflächenreaktionsbarrieren dargestellt. In den beiden Randphasen wird für das jeweils andere Element eine verschwindende Löslichkeit angenommen.

Die Grenzflächenreaktionsbarriere $\kappa=\Delta \mathrm{c} / \mathrm{j}$ beschreibt bei einer gegebenen Stromdichte $\mathrm{j}$ die Abweichung $\Delta \mathrm{c}=\mathrm{c}-\mathrm{c}_{\mathrm{eq}}$ der sich einstellenden Konzentration $\mathrm{c}$ von der dem Phasendiagramm nach $\mathrm{zu}$ erwartenden Gleichgewichtskonzentration $\mathrm{c}_{\mathrm{eq}}$. Diese Abweichung entsteht aufgrund von Barrieren, die beim Transport durch die Grenzfläche überwunden werden müssen. Solche Barrieren können durch den Wechsel der atomaren Anordnungen oder Volumenänderungen beim Durchtritt durch die Grenzfläche entstehen.

Zunächst soll die Wachstumsgeschwindigkeit der Phase $\mathrm{A}_{\beta} \mathrm{B}$ berechnet werden, die zwischen zwei schon vorhandenen Phasen $A_{\alpha} B$ und $A_{\gamma} B$ wachsen soll (vgl. Abbildung 2-2). Nebenbedingung sind eine stationäre Stromdichte $j_{\beta}$ in der Phase $A_{\beta} B$ und ein konzentrationsunabhängiger Interdiffusionskoeffizient.

Die vom Gleichgewicht abweichenden Konzentrationen $\mathrm{c}_{\mathrm{ij}}$ sind bestimmt durch:

$$
c_{\beta \alpha}=c_{\beta \alpha}^{e q}-\kappa_{\beta \alpha} j_{\beta} \text { und } c_{\beta \gamma}=c_{\beta \gamma}^{e q}+\kappa_{\beta \gamma} j_{\beta} \operatorname{mit} \kappa_{i j}=\frac{\Delta c_{i j}}{j_{\beta}}
$$

Für die Stromdichte ergibt sich durch Einsetzen der Konzentrationen und einer effektiven Grenzflächenreaktionsbarriere $\kappa_{\beta}$ folgende Darstellung:

$$
j_{\beta}=\frac{\Delta c_{\beta}^{e q}}{\kappa_{\beta}} \cdot \frac{1}{1+x_{\beta} /\left(\kappa_{\beta} \tilde{D}_{\beta}\right)}
$$

mit $\kappa_{\beta}=\kappa_{\beta \alpha}+\kappa_{\beta \gamma}, \Delta c_{\beta}^{e q}=c_{\beta \alpha}^{e q}-c_{\beta \gamma}^{e q}$.

Das Wachstum der Phase $A_{\beta} B$ läßt sich wegen $x_{\beta}=x_{\beta \gamma}-x_{\beta \alpha}$ über

$$
\begin{gathered}
\frac{d x_{\beta \gamma}}{d t}\left(c_{\beta \gamma}-c_{\gamma \beta}^{e q}\right)=j_{\beta} \text { und } \frac{d x_{\beta \alpha}}{d t}\left(c_{\alpha \beta}^{e q}-c_{\beta \alpha}\right)=-j_{\beta} \quad \mathrm{zu} \\
\frac{d x_{\beta}}{d t}=\left(\frac{1}{c_{\beta \gamma}-c_{\gamma \beta}^{e q}}+\frac{1}{c_{\alpha \beta}^{e q}-c_{\beta \alpha}}\right) \cdot j_{\beta}=G_{\beta} \cdot j_{\beta}
\end{gathered}
$$

berechnen.

$\mathrm{G}_{\beta}$ ist ein Vorfaktor, der näherungsweise als konstant angenommen werden kann. Für die Wachstumsgeschwindigkeit der Phase $A_{\beta} B$ erhält man dann:

$$
\frac{d x_{\beta}}{d t}=G_{\beta} \cdot \frac{\Delta c_{\beta}^{e q}}{\kappa_{\beta}} \cdot \frac{1}{1+x_{\beta} / \kappa_{\beta} \tilde{D}_{\beta}}
$$

Aus Gleichung (2.7) läßt sich auf zwei vorkommende Wachstumsregime schließen: 


$$
\frac{d x_{\beta}}{d t}=G_{\beta} \cdot \frac{\Delta c_{\beta}^{e q}}{\kappa_{\beta}} \quad \text { für } \mathrm{x}_{\beta} \ll \kappa_{\beta} \tilde{\mathrm{D}}_{\beta},
$$

d.h. konstante Wachstumsgeschwindigkeit bei Phasenbildung bzw.

$$
\frac{d x_{\beta}}{d t}=G_{\beta} \cdot \frac{\Delta c_{\beta}^{e q} \cdot \tilde{D}_{\beta}}{x_{\beta}} \quad \text { für } \mathrm{x}_{\beta} \gg \kappa_{\beta} \tilde{\mathrm{D}}_{\beta},
$$

d.h. das Phasenwachstum geht unter obiger Bedingung in eine parabolische Zeitabhängigkeit über und verlangsamt sich dabei mit größer werdender Dicke.

Beim Wachstum von Oxidschichten auf Silizium konnte die lineare Zeitabhängigkeit in den Anfangsstadien experimentell nachgewiesen werden [DEAL]. Physikalisch erscheint dieses Wachstumsverhalten in den Frühstadien der Interreaktion sinnvoller als das Modell des diffusionskontrollierten Wachstums, bei dem die Wachstumsgeschwindigkeit für Zeiten $\mathrm{t} \rightarrow 0$ wegen $\frac{d x_{1}}{d t} \sim \frac{1}{\sqrt{t}}$ gegen unendlich divergiert. Für $\mathrm{t} \rightarrow \infty$ folgt die Schichtreaktion parabolischem Wachstumsverhalten, das um den Betrag $-\mathrm{kD}$ auf der Dickenachse verschoben ist.

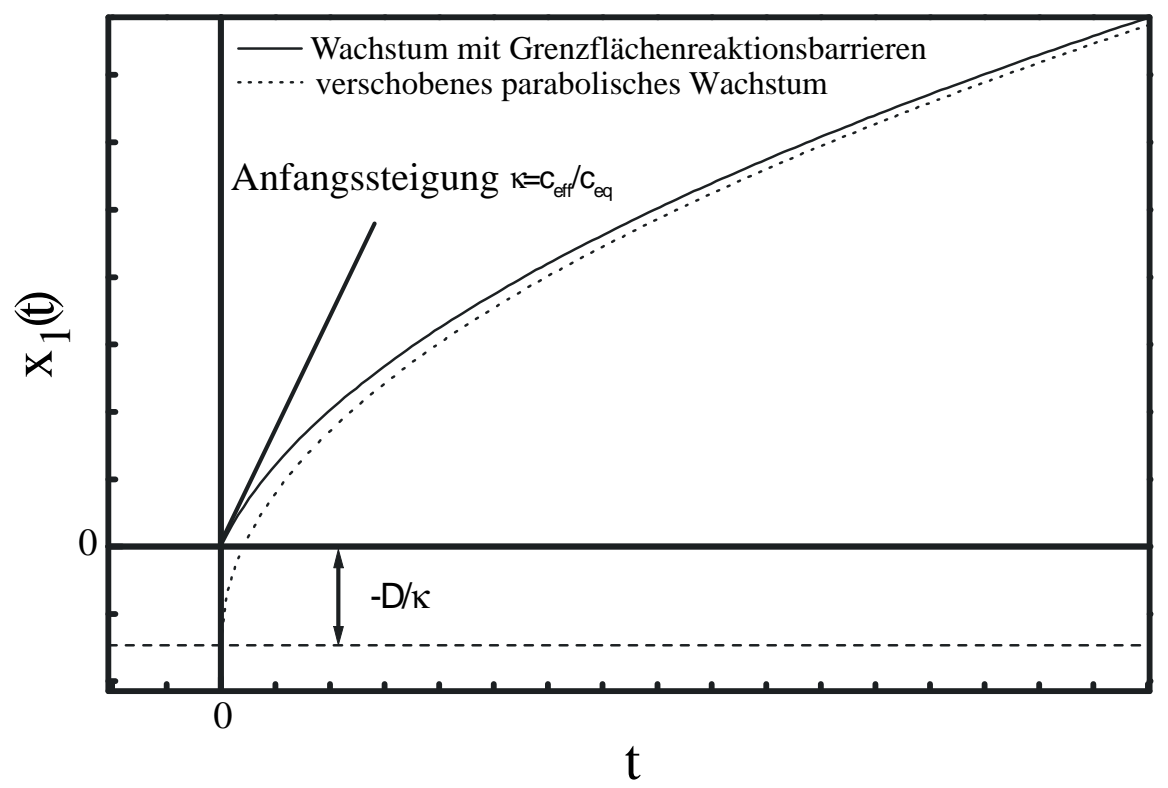

Abbildung 2-3: Darstellung des Wachstumsverhaltens in den Frühstadien und nach einer kritischen Schichtdicke $\kappa D$.

Der Übergang von grenzflächenkontrolliertem Wachstum zu diffusionskontrolliertem, parabolischem Wachstum erfolgt bei einer kritischen Schichtdicke $-\kappa \mathrm{D}$ (vgl. Abbildung 2-3). Diese liegt bei metallischen Schichtsystemen typischerweise im Bereich von wenigen Monolagen [BormanN]. Das macht deutlich, daß für einen direkten experimentellen Nachweis auf dieser Skala nur sehr wenige analytische Methoden geeignet sind. 


\subsubsection{Phasenselektion}

Die oben dargestellten Formalismen lassen sich ohne weiteres auf mehrere existente Phasen erweitern.

Ziel ist es, das Wachstum der Phase $A_{\beta} B$ in Konkurrenz mit einer ebenfalls wachsenden Phase $\mathrm{A}_{\gamma} \mathrm{B} \mathrm{zu}$ betrachten. Dies geschieht durch Definition von Zusatztermen nach obiger Vorgehensweise:

$$
\frac{d x_{\beta}}{d t}=G_{\beta} j_{\beta}-G_{\beta \gamma} j_{\gamma} \text { und } \frac{d x_{\gamma}}{d t}=G_{\gamma \delta} j_{\gamma}-G_{\gamma \beta} j_{\beta},
$$

wobei $\mathrm{G}_{\beta \gamma}, \mathrm{G}_{\gamma \beta}, \mathrm{G}_{\gamma \delta}$ und $\mathrm{G}_{\beta}$ systemabhängige Konstanten darstellen.

Damit ergeben sich für das Wachstum der beteiligten Phasen folgende Bedingungen:

$$
\begin{aligned}
& \frac{d x_{\beta}}{d t}>0 \Leftrightarrow \mathrm{r}=\frac{j_{\beta}}{j_{\gamma}}>\frac{G_{\beta \gamma}}{G_{\beta}}=\mathrm{r}_{1}=\text { const. } \\
& \frac{d x_{\gamma}}{d t}>0 \Leftrightarrow \mathrm{r}=\frac{j_{\beta}}{j_{\gamma}}<\frac{G_{\gamma}}{G_{\beta \gamma}}=\mathrm{r}_{2}=\text { const. } \quad \text { mit } r_{1}<r_{2}
\end{aligned}
$$

Die Grenzwerte $r_{1}$ und $r_{2}$ der drei Bereiche sind von den Konzentrationen der einzelnen Phasen abhängig. Wie in Abbildung 2-4 dargestellt, wachsen beide betrachteten Phasen $\beta$ und $\gamma$ nur für ein Stromdichtenverhältnis $r$ mit $r_{1}<r<r_{2}$. Außerhalb dieser Grenzen wächst die eine Phase auf Kosten der anderen. Das Wachstum einer Phase hängt demnach nicht alleine vom eigenen Volumentransport an die Grenzflächen ab, sondern zusätzlich von der Diffusion durch die Nachbarphasen. So ist verständlich, daß selbst eine stabile Gleichgewichtsphase aufgelöst werden kann, aufgrund eines viel schnelleren Wachstums ihrer Nachbarn.

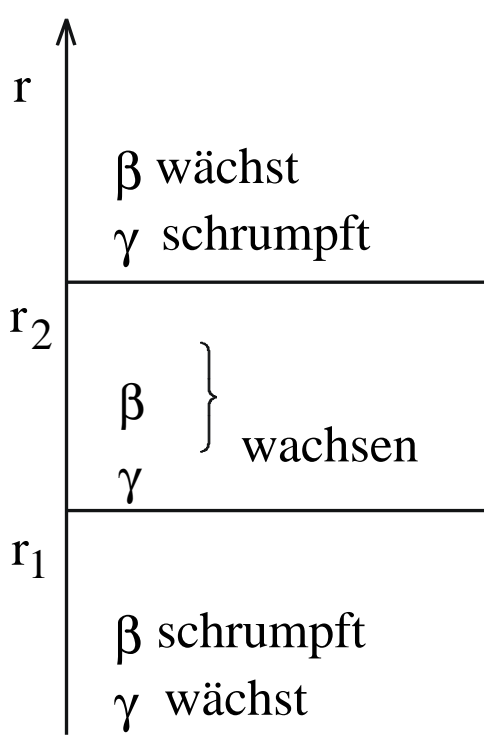

Abbildung 2-4: Darstellung der berechneten Wachstumsgebiete nach GöSELE und TU. 
Eine Phasenauswahl findet statt, wenn der Reaktionsanfang in Gebiet $r>r_{2}$ oder $r<r_{1}$ fällt und so die Bildung der zweiten Phase unterdrückt wird.

Bis zum heutigen Zeitpunkt ist die Existenz und der Einfluß einer Grenzflächenbarriere umstritten. Gerade aufgrund der sehr geringen kritischen Schichtdicke von wenigen Monolagen ist die experimentelle Bestimmung von $\kappa$ sehr schwierig.

\subsection{Keimbildungs- und Wachstumsmodell nach COFFEY}

Dem Phasenwachstum und einer eventuellen Wachstumsauslese muß zuerst eine Keimbildung vorausgehen. Ein Modell zur Behandlung der Keimbildung kommt aus experimentellen Befunden von DSC-Messungen an dünnen metallischen Schichten. Bei der Wärmebehandlung von einigen Schichtsystemen, wie z.B. Nb/Al oder $\mathrm{Ag} / \mathrm{Al}$, erscheinen zwei exotherme Wärmetönungen, die jedoch der Bildung nur einer Produktphase zugeordnet werden (vgl. Abbildung 4-2). Durch Variation der Schichtdichte verschiebt sich die Lage des zweiten Peaks, während der erste unverändert bleibt. COFFEY interpretiert das Auftreten der Doppelpeaks mit einem zweistufigen Reaktionsprozeß.

a)

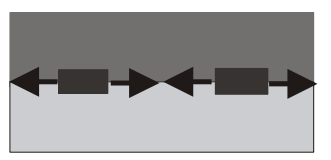

b)

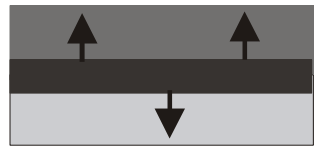

Abbildung 2-5: Zweistufiges Reaktionsmodell nach Coffey. a) Keimbildung und laterales Wachstum; b) Volumendiffusionskontrolliertes Dickenwachstum.

Im ersten Schritt kommt es zur Keimbildung und lateralem Wachstum bis zur geschlossenen Keimlage (vgl. Abbildung 2-5 a). Danach erfolgt im zweiten Schritt das Dickenwachstum, welches durch die Volumendiffusion durch die Produktschicht gesteuert wird. In diesem Modell werden zur quantitativen Berechnung der Kinetik die Keime als kleine Zylinder der Anfangshöhe $z_{0}$ angenommen. Diese wachsen radial, bis sie aneinander stoßen und die gesamte Grenzfläche bedeckt haben. Das Aneinanderstoßen der wachsenden Zylinder wird nach AVRAMI, JOHNSON und MEHL beschrieben [Avrami, Johnson3]: Der tatsächlich reagierte Anteil $\mathrm{X}_{\mathrm{A}}$ der Grenzfläche reduziert sich von der naiven Summe der Zylindergrundflächen $n \pi r^{2}$ auf

$$
X_{A}=1-\exp \left(-n \pi \cdot r^{2}\right) \text {, }
$$

mit r-Zylinderradius, n-Flächendichte der Keimbildungszentren. Zusammen mit dem diffusionsgesteuerten Dickenwachstum verhält sich der reagierte Volumenanteil $\mathrm{X}_{\mathrm{V}}$ gemäß

$$
\frac{d X_{V}}{d t}=\frac{d X_{A}}{d t} \cdot \frac{z_{0}}{z_{\max }}+X_{A} \frac{d z}{d t} \cdot \frac{1}{z_{\max }},
$$

mit $\mathrm{z}_{\max }-$ maximal erreichbare Produktschichtdicke und

$$
\frac{d X_{A}}{d t}=2 n \pi \cdot r\left(\frac{d r}{d t}\right) \exp \left(-n \pi \cdot r^{2}\right)
$$


Diese Beschreibung gibt den zweistufigen Verlauf der DSC-Kurve zwar gut wieder, jedoch sind die berechneten Keimgrößen mit einigen zehn Nanometern extrem groß, was auf zu stark vereinfachende Annahmen zurückzuführen ist. Als Kritik an dem Modell muß angemerkt werden, daß wichtige kinetische Aspekte vernachlässigt werden. Signifikant unterschiedliche Diffusionskoeffizienten gehen ebenso wenig ein, wie der Einfluß der Korngrenzendiffusion, obwohl beide die Keimbildung beeinflussen.

\subsection{Einfluß der Mikrostruktur}

Die vorher erwähnten Modelle zur Phasenbildung und -auswahl gehen auf vereinfachende kinetische und thermodynamische Betrachtungen zurück. Grundlage ist die Annahme, daß die Produktphase eine homogene geschlossene Lage bildet (vgl. Abbildung 2-6 a). Es gibt eine Reihe von Systemen bei denen dies sicherlich eine passende Beschreibung ist und in vielen Anwendungen ist planares Wachstum geradezu erwünscht.

Ebenso scheint aber gerade im Bereich metallischer Dünnschichtsysteme, die eher nanokristalline Struktur besitzen, die Annahme planarer Reaktionsgeometrie häufig auf einen Meßartefakt zurückzugehen, bedingt durch das limitierte laterale Auflösungsvermögen der häufig eingesetzten Methoden.

a)

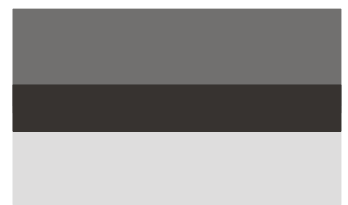

b)

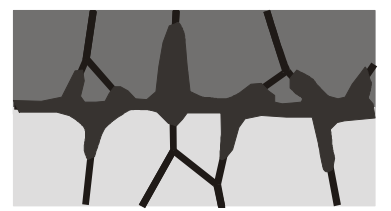

Abbildung 2-6: a) Häufig angenommenen planare Reaktionsgeometrie und b) bei dünnen metallischen Schichten eher zutreffende reale Mikrostruktur.

Teilbild b) scheint daher für diese Systeme eine realistischere Morphologie zu sein. Wie in den späteren Abschnitten der Arbeit herausgestellt wird, sind die Fehler in diesen Schichtstrukturen entscheidend für den Reaktionsablauf. Im Fall Ag/Al spielen heterogene Keimbildungszentren in Tripelpunkten von Korngrenzen eine wichtige Rolle. Ebenso sind schnelle Diffusionswege entlang der Korngrenzen für die Entwicklung einer 3D-Reaktionsmorphologie verantwortlich. Bei den untersuchten magnetischen Schichten in Kapitel 5 ermöglichen diese Schichtfehler ein Absinken des Magnetowiderstandes bei Wärmebehandlung. In der Literatur lassen sich viele weitere Beispiele für die Einfluß der Mikrostruktur auf das Eintreten und den Verlauf der Interreaktion finden. Auf dem Gebiet der Festkörperamorphisierung hat MENG anhand von gesputterten und ausgeheilten $\mathrm{Ni} / \mathrm{Zr}-\mathrm{Schichten}$ demonstriert, daß es nur zur Bildung einer amorphen Phase kommt, wenn hochenergetische Großwinkelkorngrenzen in der $\mathrm{Zr}$-Schicht vorhanden sind und als heterogene Keimzentren der amorphen Phase zur Verfügung stehen [MENG]. Im Fall einer rekristallisierten $\mathrm{Zr}$-Lage, die nur Kleinwinkelkorngrenzen besitzt, bleibt die Reaktion aus. In $\mathrm{Cu} / \mathrm{Y}$-Multischichten bildet sich die amorphe Produktphase in den Phasengrenzflächen und den Korngrenzen des Y. TiSi und TiNi sind weitere Systeme, in denen ähnliche Korngrenzenamorphisierung beobachtet 
wurde [JoHnson2]. Ein weiteres interessantes Beispiel ist die Silizidbildung am System Si-NiSi, welche eine deutliche 3D-Reaktionsmorphologie entwickelt. Die Keime der $\mathrm{NiSi}_{2}-$ Phase entstehen an der inneren Phasengrenze in den Korngrenzentripelpunkten des NiSi. Sie wachsen sehr schnell, sogar bis durch die ganze Filmdicke, bevor sie lateral zusammenwachsen [HEURLE2].

Meßbar wird der dominante Korngrenzeneinfluß durch Bestimmung der Wachstumsrate. Häufig werden Zeitexponenten gemessen die deutlich von 1, für grenzflächenkontrolliertes Wachstum, oder von 1/2, für parabolische Abhängigkeit, abweichen und einen deutlichen Hinweis auf korngrenzendiffusionsbestimmtes Wachstum geben.

Diese Beispiele zeigen, daß die Annahme einer planaren Geometrie zur Beschreibung des Reaktionsablaufes nicht immer passend ist und Abweichungen vom erwarteten Verhalten deshalb verständlich sind. Nur durch Detailkenntnis der Mikrostruktur ist eine angemessene Beschreibung der Interreaktion möglich. 


\section{Experimentelle Methoden}

Im diesem Kapitel wird zunächst die in dieser Arbeit schwerpunktmäßig angewendete Tomographische Atomsonde vorgestellt und die dafür aufwendige Probenpräparation vorgestellt. Anschließend erfolgt ein kurzer Überblick über die weiteren benutzten Analysetechniken.

\subsection{Feldionenmikroskop (FIM) und Tomographische Atomsonde (TAP)}

\subsubsection{Das Feldionenmikroskop}

Das Feldionenmikroskop (FIM) ermöglicht die Abbildung der Oberfläche einer elektrisch leitenden Probe durch Feldionisation mit einer Vergrößerung von $10^{6}-10^{7}$. Die zu untersuchende Probe hat spitzenförmige Gestalt mit einem Krümmungsradius am Apex zwischen $20 \mathrm{~nm}$ und $50 \mathrm{~nm}$. Die Präparation der Spitzen erfolgt durch elektrochemische oder speziell entwickelte Strukturierungsverfahren, die später noch eingehend beschrieben werden. In der folgenden Abbildung 3-1 ist auf der linken Seite die Funktionsweise des FIM schematisch dargestellt. Die Probenspitze befindet sich in einem UHV-Rezipienten mit einem Restgasdruck kleiner $10^{-9} \mathrm{mbar}$.

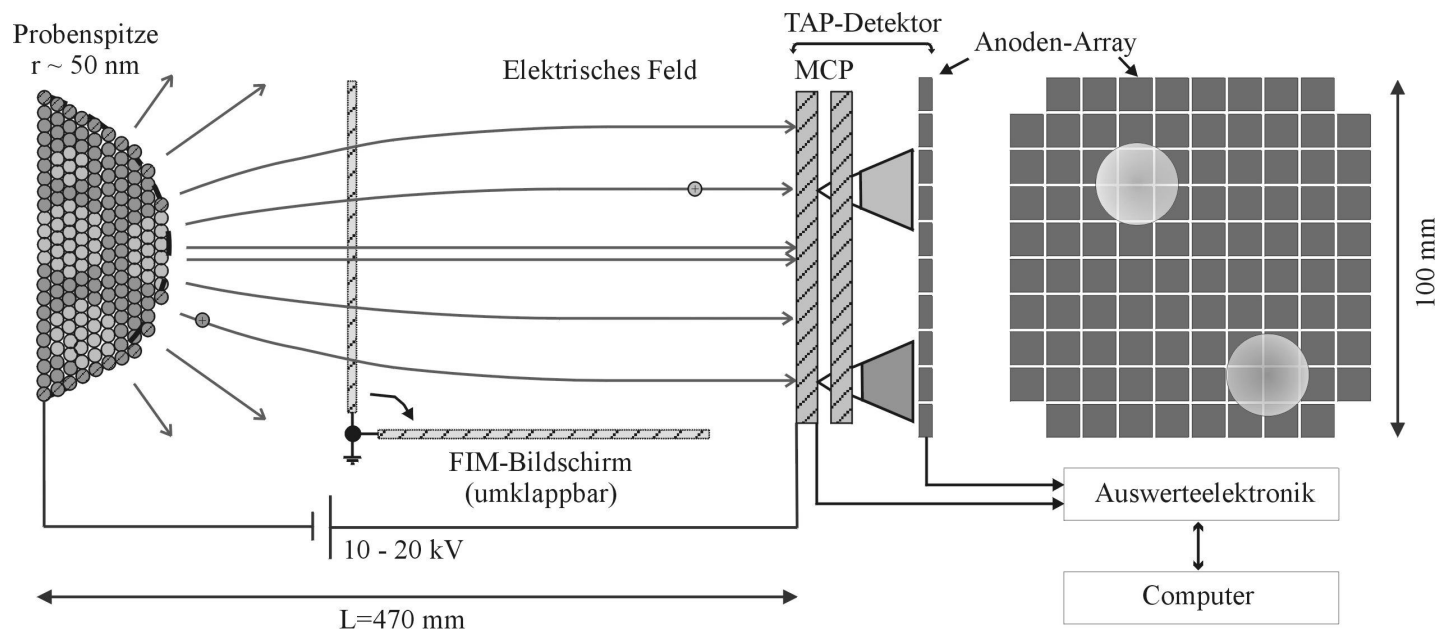

Abbildung 3-1: Schematische Darstellung des Feldionenmikroskops (linke Seite) und der Tomographischen Atomsonde (rechte Seite). Während des FIM-Betriebes ist der Bildschirm hochgeklappt und durch Kombination von eingelassenem Bildgas mit angelegter Grundspannung erfolgt die Abbildung der Oberfläche. Zur atomaren Analyse im AtomsondenModus wird der Bildschirm heruntergeklappt. Die feldevaporierten Ionen werden durch den positionsempfindlichen Detektor registriert und mit der zugehörigen Elektronik ausgelesen. Auf der rechten Seite ist das Detektorfeld in Aufsicht dargestellt. Ebenfalls sind die erzeugten Ladungswolken dargestellt, die über die Berechnung des Ladungsschwerpunktes zur Rekonstruktion des Probenvolumens dienen. 
Ihr gegenüber in einem definierten Abstand von wenigen Zentimetern befindet sich ein Bildverstärker, bestehend aus zwei Kanalplatten gefolgt von einem Fluoreszenzschirm. An die Spitze wird dann eine positive Grundspannung $U_{D C}$ von bis zu $20 \mathrm{kV}$ angelegt. Durch den kleinen Krümmungsradius der Spitze erreicht man ein zur Feldionisation notwendiges lokales Feld von über $60 \mathrm{~V} / \mathrm{nm}$ :

$$
F=\frac{U_{D C}}{k \cdot r},
$$

mit einem von der Form der Spitze abhängigen Geometriefaktor k = (4 - 9) [MÜLLER]. Zusätzlich wird die Spitze mit einem Heliumkryostaten auf eine Temperatur zwischen $20 \mathrm{~K}$ und $100 \mathrm{~K}$ abgekühlt. Auf dem Leuchtschirm erhält man ein stehendes Bild mit einer Vergrößerung zwischen $10^{6}$ und $10^{7}$ :

$$
\eta=\frac{R}{b \cdot r},
$$

mit R-Abstand Spitze-Kanalplatte, b-Bildkompressionsfaktor $(1,5$ - 1,8) [MÜLLER]. Zur Abbildung wird $\mathrm{Ne}, \mathrm{He}$ oder $\mathrm{H}_{2}$ als Bildgas mit einem Partialdruck von bis $\mathrm{zu}$ $5 \cdot 10^{-5} \mathrm{mbar}$ in den UHV-Rezipienten gegeben. Nahe der Spitze wird das Bildgasatom polarisiert (1), thermalisiert und zu Orten hoher Feldstärke gezogen. Orte extremer Feldstärke sind insbesondere Kanten- oder Adatome, an denen aufgrund großer lokaler Krümmungen die Feldstärken überdurchschnittlich hoch sind.

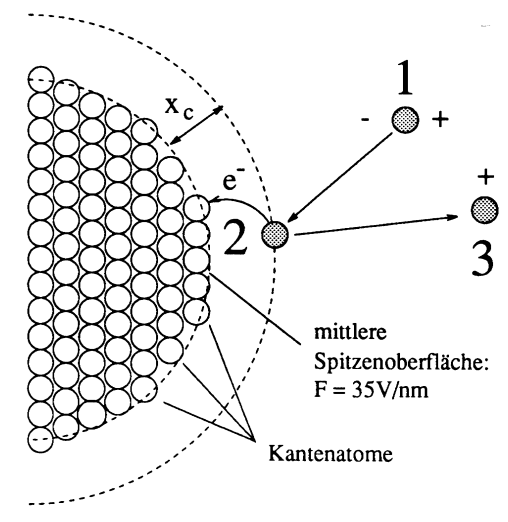

Abbildung 3-2: Feldionisation von Bildgasatomen an der Probenspitze

In einem Abstand $\mathrm{x}_{\mathrm{c}}$ wird das Bildgasatom durch einen Tunnelprozeß ionisiert (2) und radial in Richtung der Kanalplatten beschleunigt (3). Dort erzeugt der kontinuierliche Ionenstrom auf dem Leuchtschirm ein Bild, das einer nahezu stereographischen Projektion der Spitzenoberfläche entspricht.

Die in der Probe vorhandene kristallographische Orientierung läßt sich anhand der entstehenden Polstruktur ablesen. Niedrig indizierte kristallographische Richtungen sind von ringförmigen Terrassen umgeben, deren Stufen Orte hoher lokaler Feldstärke sind und deshalb als konzentrische Ringe abgebildet werden. Der Spitzenradius $r$ läßt sich über die Anzahl $n$ der Ringe zwischen zwei indizierten Polen mit Winkelabstand $\theta$ bestimmen [DRECHSLER]: 


$$
r=\frac{n \cdot d_{h k l}}{1-\cos \theta} .
$$

Das am Institut für Materialphysik in Göttingen betriebene Feldionenmikroskop wird in [GEBER] beschrieben.

Erhöht man die angelegte Spannung über die sogenannte Bestbildspannung hinaus, können einzelne Atome von der Probenoberfläche durch Feldverdampfung abgelöst werden. Auf diese Weise lassen sich gezielt Atomlage für Atomlage abtragen. Dieser Effekt wird bei der Tomographischen Atomsonde für die atomar scharfe, chemische Charakterisierung des Probenvolumens ausgenutzt.

\subsubsection{Die Tomographische Atomsonde (TAP)}

Beim Atomsondenbetrieb wird gezielt durch Anlegen eines zusätzlichen Hochspannungspulses $U_{P}$ zur Grundspannung $U_{D C}$ die Feldverdampfungsfeldstärke des Probenmaterials überschritten. Einzelne Atome verlassen ionisiert die Oberfläche und werden entlang der Feldlinien in Richtung des Detektors beschleunigt. Der Puls dient gleichzeitig als Startsignal einer Uhr, die durch Auftreffen des Ions auf den Detektor gestoppt wird. Mit der so erhaltenen Flugzeit t läßt sich aufgrund der bekannten Fluglänge d und der kinetischen Energie der Ionen auf die spezifische Masse der Ionen schließen [MILLER]:

$$
\frac{m}{n}=\frac{2 e}{d^{2}} \cdot\left(U_{D C}+U_{P}\right) \cdot t^{2},
$$

wobei $\mathrm{n}$ der Ionisationsgrad ist. Am Institut für Materialphysik in Göttingen kommen zwei verschiedene Detektorarten zum Einsatz. Zum einen der konventionelle eindimensionale Detektor und zum anderen das neuartige positionssensitive Detektorarray der 3D-Atomsonde (TAP). Bei der klassischen Atomsondenmessung fliegen die feldverdampften Ionen durch eine kleine Apertur in den als Bildschirm genutzten Kanalplatten. Die auf diese Apertur projizierte Probenoberfläche liegt bei etwa 2-5 nm im Durchmesser. Über diese Fläche wird die laterale chemische Information summiert. In Meßrichtung ist die Auflösung aber definiert durch das sukzessive Abtragen der Atomlagen. Das bedeutet eine Tiefenauflösung von etwa $0,2 \mathrm{~nm}$ und in laterale Richtung 2-5 nm. Mit anderen Worten, die Information woher das Ion aus der atomaren Lage kommt, kann nicht aufgelöst werden.

Die an der Universität Rouen (Frankreich) [BLAVETTE2, BOSTEL] entwickelte und auch in Göttingen zum Einsatz kommende Tomographische Atomsonde (vgl. Abbildung 3-1) verfügt über ein ortsempfindliches Detektorarray und ermöglicht eine atomare Rekonstruktion des gemessenen Probenvolumens. Durch das auf die erste Multichannelplate (MCP) auftreffende Ion wird eine Elektronenkaskade ausgelöst, die zunächst als Stopsignal für die Flugzeitmessung dient. Die nachgeschalteten MCP verstärken die Elektronenkaskade weiter. Es entsteht eine Elektronenwolke, die auf das dahinter befindliche positionssensitive Detektor-Array trifft. Der Detektor besteht aus 96 Anoden auf einer Fläche von $10 \cdot 10 \mathrm{~cm}^{2}$. Die jeweilige auf den einzelnen Anoden deponierte Ladung wird über AD-Wandler ausgelesen. Für eine gute Schwerpunktbestimmung [BLAVETTE1] sollte die Elektronenwolke mindestens 
3-4 Anodenfelder überstreichen. Die Elektronik ist in der Lage auch mehrere auftreffende Ionen mit gleichen Massen und dementsprechend gleichen Flugzeiten zu detektieren und auszuwerten. Voraussetzung ist allerdings, daß die erzeugten Elektronenwolken nicht überlappen. Über die genaue Bestimmung des IonenEinschlagortes auf dem Detektor läßt sich mit Kenntnis der Flugbahn der Atomort im analysierten Probenvolumen rekonstruieren. Die lateral analysierte Fläche ist bei diesem System wesentlich größer als bei der konventionellen Atomsonde. Typischerweise wird ein Probenvolumen von $15 \cdot 15 \cdot 100 \mathrm{~nm}^{3}$ analysiert. Die laterale Auflösung wird eingeschränkt durch ungenaue Bestimmung des Ladungsschwerpunktes, Abweichungen von der angenommenen Flugbahn und auch von lokalen chemischen Inhomogenitäten. Die unterschiedlichen Feldverdampfungsfeldstärken der Elemente bei Schichtsystemen oder Legierungen, können zum bevorzugten Ablösen einer Komponente und dadurch zu sogenannten Fokussierungsoder Defokussierungseffekten führen. Unter optimalen Bedingungen läßt sich aber die laterale Position auf eine Genauigkeit von $0,5 \mathrm{~nm}$ angeben. Die Tiefenauflösung ist wie bei der konventionellen Atomsonde durch das sukzessive Abtragen der Atomebenen bestimmt und liegt bei 0,2 nm. Diese Daten machen deutlich, daß diese noch sehr junge Methode ein noch nicht ausgeschöpftes Potenzial birgt. Keine andere analytische Methode erlaubt Aussagen über die chemische Struktur auf atomarer Skala in derartiger Auflösung.

Zur 3D-Rekonstruktion des Probenvolumens bedient man sich des gespeicherten Datenfiles der Analyse. Es enthält unter anderem die notwendigen Informationen wie Flugzeit t, Einschlagort (x,y), Grundspannung $U_{D C}$ und die Pulsspannung $U_{P}$. Eine ausführliche Darstellung der Rekonstruktion enthält [AL-KASSAB]. Die wichtigsten Punkte werden hier aber kurz dargestellt. Zunächst wird wie bereits erläutert mit Hilfe der Flugzeit die spezifische Masse $\mathrm{m} / \mathrm{n}$ des detektierten Ions bestimmt. Im zweiten Schritt erfolgt die Rückprojektion des Teilchens auf die laterale Position auf der Probe. Dies geschieht mittels einfacher Punktprojektion unter der vereinfachten Annahme eines kugelförmigen Spitzenapex und auslaufenden Schaft mit konstantem Winkel. Das lagenweise Abtragen entlang der Probenachse (z-Richtung) wird durch Zuordnung eines z-Intervalls pro Atom berücksichtigt, in das unter anderem das Atomvolumen, die Detektoreffizienz, dessen Fläche und der Spitzenradius eingeht. Letzterer wird in dem ursprünglich mitgelieferten Software-Modul aus dem Quotienten der angelegten Spannung $\mathrm{U}_{\mathrm{DC}}+\mathrm{U}_{\mathrm{P}}$ und der materialspezifischen Feldverdampfungsfeldstärke $\mathrm{F}_{\mathrm{E}}$ berechnet:

$$
R=\frac{U_{D C}+U_{P}}{F_{E} \cdot \beta} .
$$

Der Geometriefaktor $\beta$ berücksichtigt die Abweichung von der Kugelgeometrie und liegt zwischen 5 und 10. Diese Rekonstruktion setzt allerdings eine konstante Verdampfungsfeldstärke der enthaltenen Materialien voraus. Bei der Analyse von heterogenen Schichtsystemen führt dieser Ansatz jedoch zu verzerrten Volumenrekonstruktionen in Bereichen unterschiedlicher Elemente. Ist eine Schicht mit niedriger Feldverdampfungsfeldstärke auf einem Material mit hoher Feldver- 
dampfungsfeldstärke aufgebracht, so kommt es bei der Rekonstruktion zur Ausbildung sogenannter „bottle neck“-Strukturen. Ein gutes Beispiel sind die in [JESKE1] gezeigten rekonstruierten $\mathrm{Al} / \mathrm{Ni}-\mathrm{Schich}$ ten. Der Hauptteil der in dieser Arbeit vorgestellten Untersuchungen betrifft aufgebrachte Schichtsysteme auf Trägerspitzen. Gerade das System $\mathrm{Al} / \mathrm{Ag}-\mathrm{W}$ ist ebenfalls ein Paradebeispiel für die erwähnte ,bottle neck“-Bildung aufgrund sehr unterschiedlicher Feldverdampfungsfeldstärken.

Zum Zwecke einer vernünftigen Rekonstruktion wurde von SCHMITZ ein alternativer Algorithmus vorgeschlagen [JESKE1], der die geometrische Gestalt der Spitze als Ausgangsparameter hat. Anstatt aus Gl. (3.5) wird der momentane Spitzenradius bestimmt über

$$
\frac{d R}{d z}=\frac{\sin \gamma}{1-\sin \gamma}
$$

Es gehen der anfängliche Spitzenradius R und der Schaftwinkel $\gamma$ der Spitze ein. Ein Ergebnis aus diesem Ansatz und dem Vergleich mit Gl. (3.5) ist das Produkt $F_{E} \bullet \beta$. Ein Vergleich dieses Produkts mit theoretischen Werten und vorher durchgeführten Eichmessungen erlaubt die Qualität der Rekonstruktion zu beurteilen.

Für die Wahl der richtigen Größen der Parameter Spitzenradius R und Schaftwinkel gibt es eine Reihe von zusätzlichen hilfreichen Informationen. Zum einen geben TEM-Bilder von der Probe die aufgebrachten Schichtdicken und entsprechend Radius und Schaftwinkel wieder. Des weiteren gibt die Kontrolle des rekonstruierten Volumens weiteren Aufschluß. Im Regelfall lassen sich Bereiche finden in denen deutlich Netzebenen zu erkennen sind. Dies kann im Schichtpaket sein oder auch in der Substrat-Trägerspitze.

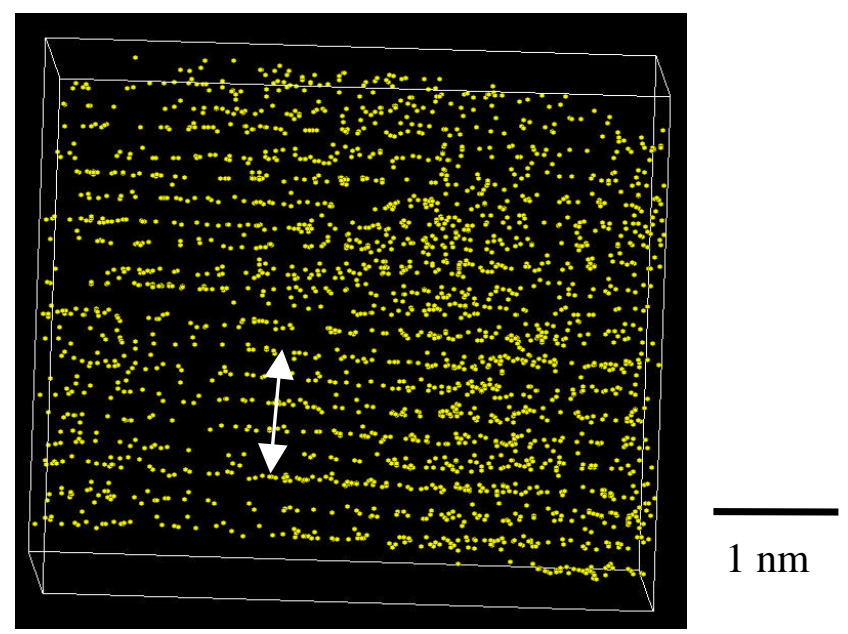

Abbildung 3-3: Rekonstruktion von $\mathrm{Ni}-$ Netzebenen.

Sind diese Netzebenen nicht eben oder stimmt der Netzebenenabstand nicht mit Literaturwerten überein, so müssen die Parameter solange optimiert werden, bis dieses der Fall ist. Ein Beispiel für eine gelungene Rekonstruktion ist in Abbildung 3-3 dargestellt. Gezeigt sind die Netzebenen einer gesputterten Ni-Schicht. Die 
Probe wurde entlang der Wachstumsrichtung, für kubischflächenzentriertes Ni [111], analysiert. Die Parameter wurden solange optimiert, bis die rekonstruierten Netzebenen den entsprechenden Abstand $d_{111}=\frac{a}{\sqrt{3}}$, mit a $=3,53 \AA$ [KITTEL], wiedergeben. In der Darstellung sind 5 Netzebenen mit jeweiligem Abstand $\mathrm{d}=2,04 \AA$ markiert.

\subsubsection{Datenanalyse}

Die Analyse der aufgenommenen Daten geschieht in Göttingen auf einer Digital Personal Workstation 433. Zur Visualisierung und Analyse der Daten wird das Programm AVS, Version 5.3, von Advanced Visual Systems Inc. benutzt. Es bietet die Möglichkeit die nötigen Tools, die in Rouen (Frankreich) und Göttingen für die Datenanalyse entworfen wurden modular einzubinden. Neben der einfachen 3DRekonstruktion der Daten lassen sich verschiedenste Analysen durchführen, um die gemessenen Nanostrukturen zu charakterisieren.

3D-Konzentrationen. Zur Darstellung eines Konzentrationsverlaufes im dreidimensionalen wird das $\mathrm{zu}$ untersuchende Volumen in Würfel mit der Kantenlänge 1,5 nm zerteilt, wobei diese typischerweise mit einer Schrittweite von $0,5 \mathrm{~nm}$ ineinander verschachtelt sind. Bei der Größe der Quader ist auf eine genügende Anzahl an Atomen für eine vernünftige Statistik zu achten. In diesen Würfeln wird nun die jeweilige Konzentration bestimmt und entsprechend als Isokonzentrationsfläche dargestellt. Eine weitere Möglichkeit ist die Darstellung der Konzentration auf ebenen Schnitten, wobei der gewünschte Atomsortengehalt mit einer Farbskala kodiert wird.

Konzentrationsprofile. Dank der 3D-Rekonstruktion der Meßdaten lassen sich Konzentrationsprofile an beliebiger Stelle des Probenvolumens erstellen. Mit einem frei justierbaren Zylinder wird dabei das zu untersuchende Volumen markiert. Innerhalb dieses Analysezylinders rastert eine Scheibe konstanter Dicke überlappend das Volumen ab. Die so ermittelten Konzentrationen werden dann über die Meßtiefe aufgetragen. Zur Berücksichtigung von statistischen Schwankungen wird zu den Konzentrationen immer ein Fehlerintervall angegeben. Üblicherweise werden die $2 \sigma-$ Grenzen berechnet, so daß mit einer Signifikanz von $95 \%$ darüber entschieden werden kann, ob gemessene Konzentrationsschwankungen statistisch oder real bedingt sind:

$$
\sigma=\sqrt{\frac{c(1-c)}{N-1}} .
$$

Da das Testvolumen festgelegt ist, schwankt die Anzahl der enthalten Atome. Diese lokalen Dichteschwankungen haben mehrere Gründe. Zum einen werden durch die Detektoreffizienz nur $50 \%$ der Ionen detektiert. Zum anderen führt lokal unterschiedliche Feldverdampfung zum Beispiel an Korngrenzen zu möglichen 
Dichteschwankungen. Dementsprechend muß das Blockvolumen gewählt werden, um über eine genügende Anzahl an enthaltenen Atomen eine gute Statistik zu gewährleisten. Durch geeignete Anpassung des Zylinders an das zu untersuchende Volumen ist immer eine aufgabengerechte Analyse möglich.

$\chi^{2}$-Test. Bei den untersuchten Proben steht man oft vor dem Problem festzustellen, ob die Komponenten homogen verteilt sind oder sich schon Entmischungstendenzen zeigen. Diese Frage läßt sich nicht durch visuelle Beurteilung des Probenvolumens oder durch Konzentrationsprofile eindeutig beantworten, insbesondere, wenn die Signifikanz nicht deutlich ist.

Zur Bearbeitung solcher Fragestellungen bedient man sich eines allgemein bekannten Test der mathematischen Statistik. Der $\chi^{2}$-Test prüft die Hypothese, ob eine Zufallsgröße einem vorgegebenen Verteilungsgesetz genügt [MILLER, THUVANDER, BRONSTEIN]. Entsprechend der aufgenommenen TAP-Daten, werden Stichproben mit konstanter Anzahl an Atomen dem Meßvolumen entnommen. Das Raster der Entnahmestellen ist dabei so gewählt, daß sich die Stichproben nicht oder nur unwesentlich überlappen. Auf diese Weise ist die Unabhängigkeit der Stichproben, die in der Regel zwischen 50 und 200 nächste Nachbaratome umfassen, sichergestellt. Als Ergebnis erhält man eine Häufigkeitsverteilung der bezogenen Atomsorte, die als Grundlage zur Berechnung des $\chi^{2}$-Tests herangezogen wird. Zur Prüfung der Homogenität der Probe wird die gemessene Häufigkeit nun verglichen mit der Binomialverteilung. Für eine homogene Legierung mit der Zusammensetzung $A_{c} B_{(1-c)}$ ist die Wahrscheinlichkeit in einer Stichprobe aus $j$ Atomen darin i A-Atome zu finden gegeben durch:

$$
p_{i}^{(j)}=\left(\begin{array}{c}
j \\
i
\end{array}\right) \cdot c^{i} \cdot(1-c)^{j-i}, \quad(i=0,1, . ., j) .
$$

Wichtig für die weitere Berechnung ist eine geeignete Klasseneinteilung. Dabei gelten folgende Randbedingungen:

a) $\mathrm{N} \cdot \mathrm{p}_{\mathrm{i}} \geq 1$ für die Randklasse und

b) $\mathrm{N} \cdot \mathrm{p}_{\mathrm{i}} \geq 5$ für die anderen Klassen.

$\mathrm{N}$ ist die Gesamtzahl der erhobenen Stichproben und $\mathrm{p}_{\mathrm{i}}$ die Wahrscheinlichkeit, daß das Ergebnis einer Stichprobe in der Klasse i zu finden ist. Der vergleichende $\chi^{2}-$ Wert berechnet sich dann aus:

$$
\chi^{2}=\sum_{i=1}^{k} \frac{\left(M_{i}-N \cdot p_{i}\right)^{2}}{N \cdot p_{i}},
$$

abhängig von der Anzahl der Klassen $\mathrm{k}$ und der entsprechend in der Klassen auftretenden Häufigkeit $M_{i}$. Um eine Aussage über die Signifikanz des Testes zu treffen, wird dieser Wert verglichen mit einem kritischen Wert $\chi^{2}(\alpha, r)$, der üblicherweise mit $\alpha=0,05$, also einem Signifikanzniveau von $95 \%$, angegeben wird. $\mathrm{r}=\mathrm{k}-1$ ist in diesem Fall die Zahl der Freiheitsgrade. Liegt der gemessene $\chi^{2}-$ Wert unterhalb des kritischen Wertes, sind die Abweichungen von der 
Binomialverteilung also klein. Das gemessene Probenvolumen ist demnach eher homogen - oder falls $\chi^{2}$ größer als $\chi_{\alpha}{ }^{2}$, ist die Probe signifikant inhomogen.

Bestimmung des Korngrenzen-Exzess. Für die Beschreibung von Segregationsprozessen ist die absolute Menge des Materials, das sich in oder an einer Korngrenze angereichert hat, von besonderem Interesse. Die Überschuß AtomAnzahl der untersuchten Komponente $\mathrm{N}_{\text {seg }}$ Atomsorte in dem angereicherten Gebiet bezogen auf die Größe der belegten Korngrenzfläche A, definiert den Korngrenzen-Exzess $\Gamma_{0}$ :

$$
\Gamma_{0}=\frac{N_{\text {seg }}^{\text {Atomsorte }}}{A} .
$$

Die Untersuchungen mit der Tomographischen Atomsonde ermöglichen das direkte Abzählen der segregierten Atome und somit eine unmittelbare Bestimmung des Korngrenzen-Exzess. Zur Analyse eines ausgewählten Volumenbereiches wird ein Zylinder mit $2 \mathrm{~nm}$ Radius senkrecht zur Grenzfläche ausgerichtet, die durch eingefügte Isokonzentrationsflächen hervorgehoben wurde. Die erhaltenen Daten lassen sich als einfaches Konzentrationsprofil oder in Form eines Leiterdiagramms auftragen. In der Leiterdiagramm-Auftragung wird die Atom-Anzahl der untersuchten Komponente über die kumulierte Atomzahl aller Atomsorten aufgetragen. Die lokale Steigung der Kurve entspricht der lokalen Konzentration im dazugehörigen Meßvolumen. Der so ermittelte Gehalt an gesuchten Atomen in oder an der Korngrenze wird in Beziehung zur Grundfläche $A=\pi \cdot(2 \mathrm{~nm})^{2}$ des Analysezylinders gesetzt. Unter Berücksichtigung der Detektoreffizienz von 0,5, d.h. die reale Atom-Anzahl ist doppelt so hoch, läßt sich dann direkt der Korngrenzen-Exzess bestimmen.

\subsection{Herstellung von TAP-Proben durch Ionenstrahlsputterdeposition}

Bereits aus früheren Arbeiten [LANG] zur Untersuchung dünner Schichten auf W-Spitzen ist bekannt, daß eine Reinigung der Substratoberfläche direkt vor der Schichtdeposition unerläßlich für mechanisch stabile Proben ist. Im Rahmen dieser Arbeit wurde eine spezielle Beschichtungsanlage aufgebaut, die eine Reinigung der Oberfläche direkt vor dem eigentlichen Beschichtungsvorgang ohne Bruch des Vakuums ermöglicht. Abbildung 3-4 zeigt ein Schema der Beschichtungsanlage mit den wichtigsten Komponenten. Das Aufbringen der Schichten erfolgt mit einer $3 \mathrm{~cm}$ Kaufmann-Ionenquelle durch Sputterdeposition. Die Kaufmannquelle zeichnet sich durch ihre Ionengitteroptik aus, welche die Argon-Ionen aus dem inneren Plasma extrahiert. Dadurch lassen sich Strahlstrom, Energie der Ionen und Strahldivergenz wie erwünscht justieren. Ein nachgeschalteter, stromdurchflossener W-Draht dient als Neutralisator, um auch Sputtern mit Neutralteilchen zu ermöglichen. Mit der Quelle lassen sich maximale Beschleunigungsspannungen von $1,2 \mathrm{kV}$ und Strahlströme bis $100 \mathrm{~mA} / \mathrm{cm}^{2}$ realisieren. 


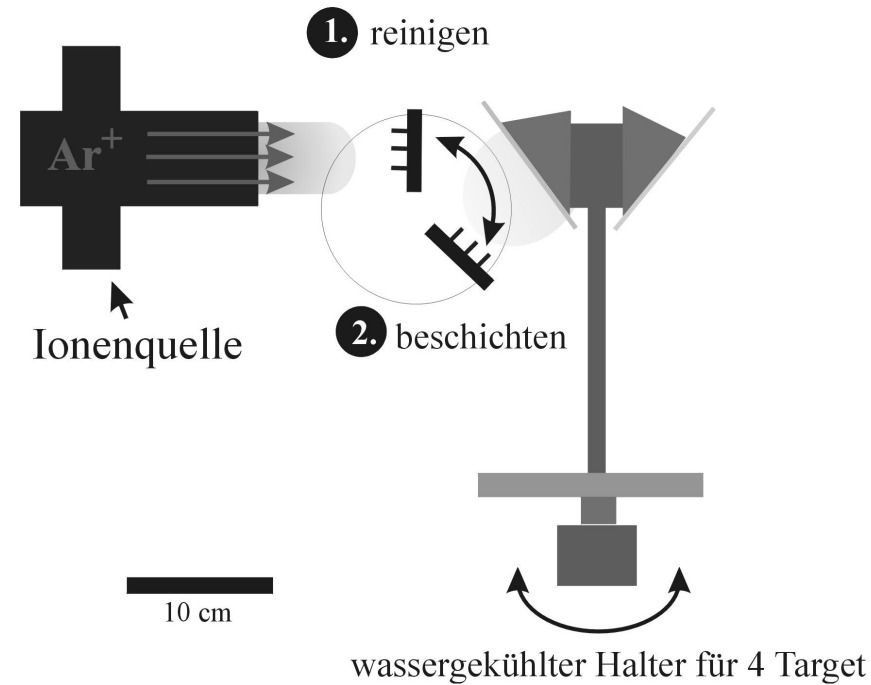

Abbildung 3-4: Schematischer Aufbau der Beschichtungsanlage - Position 1: Reinigung der Substrat-Spitzen; Position 2: Beschichtung der Spitzen

Die zu beschichtenden Substrate, präparierte Spitzen oder planare Substrate, befestigt an einem differentiell evakuierbaren Rotationsflansch, werden in Schritt (1) senkrecht zum Ionenstrahl orientiert und die Oberfläche mit dem entsprechenden Ionenstrahl ${ }^{2}$ abgesputtert. Anschließend erfolgt die Target-Reinigung, während die Substrate aus dem Strahl herausgedreht und durch einen Shutter abgedeckt sind. Zur Beschichtung (Position 2) werden die Substrate nahezu parallel zu den Targets orientiert. Die Depositionsrate läßt sich mit einer Schwingquarzwaage sehr genau von $1 \mathrm{~nm} /$ Sekunde bis auf 0,01 nm/ Sekunde kontrollieren.

\subsection{Schichtherstellung der planaren $[\mathrm{Co} / \mathrm{Cu} / \mathrm{Py} / \mathrm{Cu}]_{\mathrm{N}}-\mathrm{Systeme}$}

Die magnetischen Schichtsysteme auf planaren Substraten wurden von Heitmann und Dr. Hütten aus unserer Kooperationsgruppe der Universität Bielefeld unter der Leitung von Prof. Reiss hergestellt. Bei solchen GiantMagnetoResistanceSchichtsystemen (GMR) wechseln sich magnetische und nicht-magnetische Lagen $\mathrm{ab}$, wobei die nicht-magnetische Schicht nur wenige Nanometer dünn sein darf, um ein Koppeln der Lagen zu ermöglichen. Deswegen sind an den Herstellungsprozeß hohe Anforderungen bezüglich der Reproduzierbarkeit und der Schichtqualität gestellt.

Die Schichtherstellung erfolgte in einer Sputteranlage der Firma Leybold, Typ L560, bestehend aus einem Hochvakuum-Rezipienten mit vier DC-Magnetron-Sputterquellen. Der Basisdruck liegt bei etwa 4,6・10 $\mathrm{mbar}$. Nach dem Vorsputtern der Targets bei höherem Ar-Partialdruck werden die Schichten bei einem Druck $\leq 1,4 \cdot 10^{-3}$ mbar abgeschieden.

Vor dem Beschichten wurden die passivierten Si-Substrate im Ultraschallbad nacheinander in Aceton und Ethanol gereinigt. Näheres zur Schichtherstellung ist in [HeITMANN1] beschrieben.

\footnotetext{
${ }^{2}$ Die jeweiligen Parameter befinden sich bei der Beschreibung der hergestellten Schichtsysteme.
} 


\subsection{Lithographische Präparation planarer Schichten für die Tomographische Atomsonde}

Die geeignete Probenpräparation ist für die Tomographische Atomsonde von großer Bedeutung. Wie in Abschnitt 3.2 dargestellt, wurde im Rahmen dieser Arbeit eine Beschichtungsanlage aufgebaut, die eine Reinigung der Spitzenoberfläche direkt vor dem Aufbringen der Schichten in-situ erlaubt, ohne das Vakuum zu brechen. Allerdings wird von Kritikern der Methode gerade diese „exotische“ kugelschalenförmige Substratunterlage im Vergleich $\mathrm{zu}$ den sonst verwendeten planaren Substraten als Nachteil angeführt. Als Hauptargument wird die vermeintlich nicht vergleichbare Mikrostruktur eingebracht. Sicherlich ist die Korngrenzendichte und die Anzahl an Versetzungen in Schichten auf einer gekrümmten Spitzenoberfläche höher als auf planar aufgebrachten Schichtpaketen. Dabei darf man aber nicht unbedacht lassen, daß die untersuchten Bereiche $8-15 \mathrm{~nm}$ Kantenlänge auf einer Spitze mit dem Krümmungsradius von $50 \mathrm{~nm}$ haben. Auf der untersuchten Längenskala ist der Einfluß der Substratkrümmung sicherlich zu vernachlässigen. Aber dennoch ist die Verwendung planarer Schichtpakete für die Untersuchung in der Tomographischen Atomsonde höchst interessant, da diese Schichten aus einem Herstellungsprozeß mit verschiedenen Meßmethoden charakterisiert und anschließend atomar genau analysiert werden können. So ist zum Beispiel ein direkter Vergleich der in dieser Arbeit vorgestellten Meßergebnisse der magnetischen Schichtsysteme aus XRD- und TEM-Experimenten mit Analysen am TAP möglich.

Zur Durchführung dieses Vergleiches und um Aufschluß über den Einfluß der unterschiedlichen Substrate auf die Schichtreaktionen zu gewinnen, wurde deshalb die bereits im Rahmen der Diplomarbeit [SCHLEIwIES2] entwickelte Präparationstechnik für die Herstellung von Spitzen aus planaren Schichten angewandt. Diese beruht auf Vorarbeiten von [HoNo] und [PuNDT]. Bei [SCHLEIwIES2] findet sich eine genaue Beschreibung der Präparationsschritte. An dieser Stelle soll deshalb nur ein kurzer Abriß anhand Abbildung 3-5 über die wichtigsten Punkte gegeben werden. Bei der elektronenstrahllithographischen Strukturierung werden in einer aufgeschleuderten elektronenempfindlichen Resistschicht chemische Bindungen durch Elektronenbeschuß in dem Elektronenstrahllithographiesystem LION LV1 [SCHLIEBE] zerstört. Mit Hilfe eines geeigneten Entwicklers lassen sich selektiv die belichteten Stellen herauslösen. Auf diese Weise kann man in dem Elektronenresist eine nanostrukturierte Maske erzeugen. Durch Sputtern senkrecht auf die Oberfläche mit einer Kaufmann-Ionenquelle läßt sich die Maske in das Schichtsystem übertragen. Eine auf das Substrat aufgebrachte Pufferschicht, in diesem Fall eine $200 \mathrm{~nm}$ dicke Al-Schicht, die sich leicht in 4 molarer $\mathrm{NaOH}-$ Lösung zersetzt, ermöglicht das Ablösen der strukturierten Spitzen. 


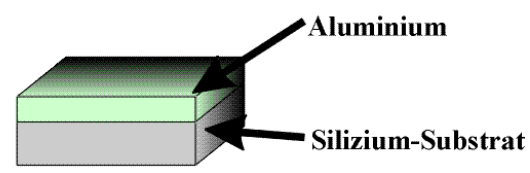

1. Aufbringen einer Pufferschicht
Multilagenpaket
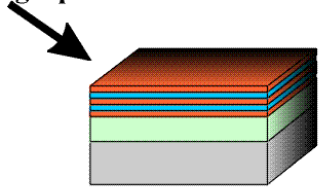

2. Aufsputtern des Multilagenpaketes

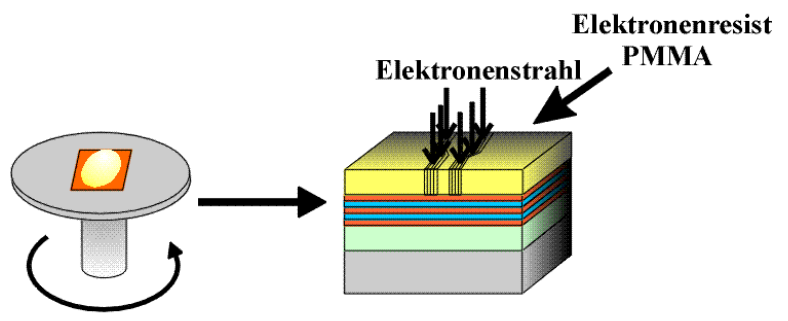

3. Aufschleudern des Elektronenresist PMMA und Belichten

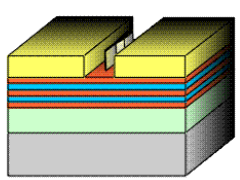

4. Entwickeln des Resist

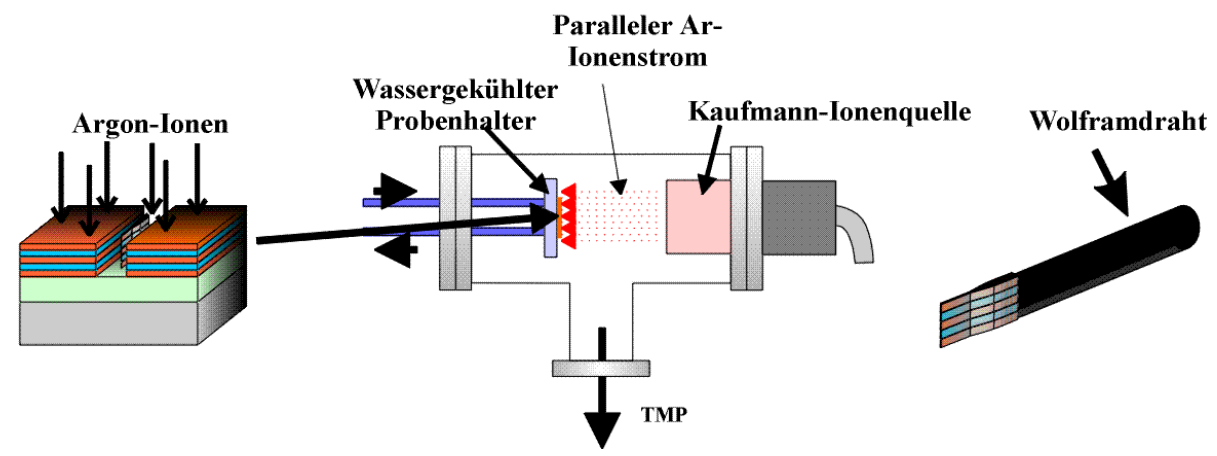

5. Übertragen der Struktur

6. Aufkleben der Spitze und Nachdünnung

Abbildung 3-5: Darstellung der Präparationsschritte zur Herstellung lithographisch strukturierter Spitzen [SCHLEIWIES1]. 
Der Puffer wurde so gewählt, daß das Lösungsmittel nicht das zu untersuchende Schichtpaket angreift. Die abgelösten Spitzen werden dann mit einem Haftmittel an einem vorbereiteten $\mathrm{Cu}$-Draht unter Zuhilfenahme eines Lichtmikroskops befestigt. Mit der Elektronenstrahllithographie lassen sich Strukturen mit Breiten unterhalb von $10 \mathrm{~nm}$ verwirklichen. Durch den anschließenden Sputterprozeß zum Strukturübertrag kann sich die Form allerdings vergröbern, so daß nach dem Aufkleben auf den $\mathrm{Cu}-$ Draht eine weitere Schärfung der Spitzen im fokussierten Ionenstrahl erforderlich werden kann.

\subsection{Präparation von Spitzen mit dem SekundärIonenMassenSpektrometer (SIMS)}

Mit dem TOF-SIMS IV steht eine weitere Methode zur Charakterisierung von Schichten mit Hilfe der Time-of-Flight Analyse am Institut für Materialphysik in Göttingen zur Verfügung. Die eigentliche Messung erfolgt durch Beschuß der Probenoberfläche mit Ga-Ionen einer Energie von $11-25 \mathrm{keV}$. Beim Auftreffen der Primärionen auf die Probe werden Probenatome durch ausgelöste Stoßkaskaden herausgeschlagen. Die Masse der ionisierten Teilchen wird ähnlich der Atomsondenanalyse aufgrund ihrer Flugzeit zwischen Primärionenpuls und Auftreffen am Analysator bestimmt. Durch eine Ablenkeinrichtung ist es möglich mit dem fokussierten Ga-Ionenstrahl eine Fläche bis zu $500 \cdot 500 \mu \mathrm{m}^{2}$ abzurastern. Diesen ablenkbaren Ionenstrahl macht man sich beim Nachschärfen der lithographierten Spitzen zu nutze. Dabei haben sich zwei Vorgehensweisen als erfolgreich erwiesen:

Methode 1. Abbildung 3-6 zeigt die lithographisch strukturierte Spitze in einem Sekundärelektronenbild abgerastert mit dem Ga-Ionenstrahl. Die Energie der GaIonen wurde auf den maximalen Wert von $25 \mathrm{keV}$ eingestellt.
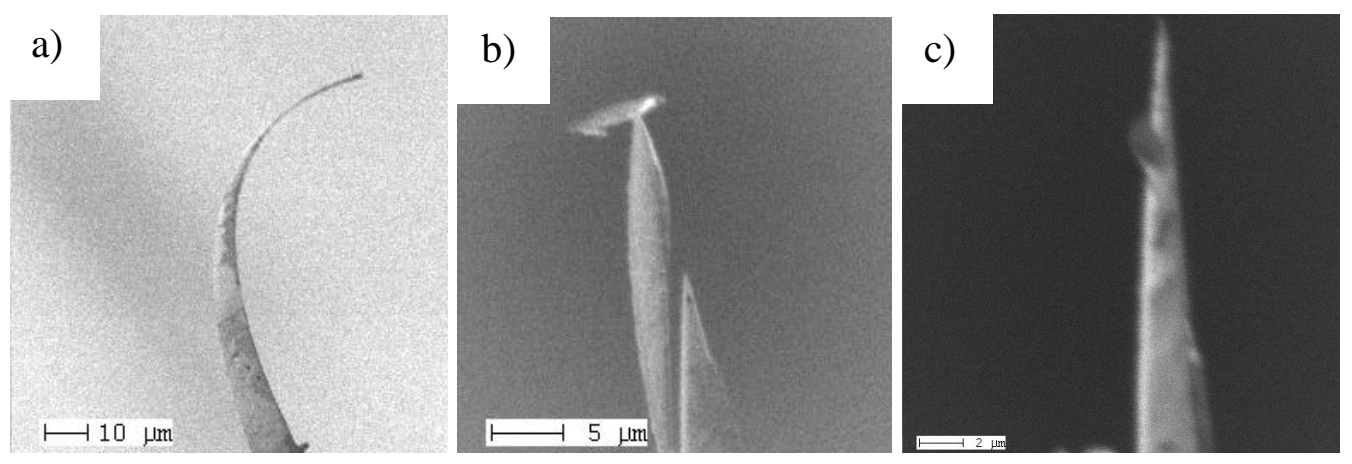

Abbildung 3-6: Schärfung von lithografisch strukturierten TAP-Proben mit dem fokussierten Ionenstrahl. a) Ausgangszustand; b) nach wenigen Schnitten; c) analysierbare Spitze.

Das eigentliche Anschneiden der Spitze erfolgt durch Abschalten der $\mathrm{x}$-Ablenkung des Ionenstrahls, so daß die genau positionierte Spitze entlang der y-Achse des Fadenkreuzes beschossen werden kann. Während des Schneidvorganges sollte die maximale Pulsdauer benutzt werden (Aktivierung der Einstellung „DC“), um die Schneidzeiten kurz zu halten. Diese Einstellung sollte aber unbedingt beim Abbilden der Spitze zwecks Neupositionierung deaktiviert werden! Aufgrund der starken 
Abtragrate besteht zum einen die Gefahr des „Wegdünnens“ der Spitze und zum anderen einer zu starken Ionenmischung, die die präparierten Schichtstrukturen zerstören könnte. Einige durchgeführte Schnitte auf dem Weg zur benutzbaren Spitze sind in Abbildung 3-6 zu sehen.

Methode 2. Eine weitere Möglichkeit des Nachschneidens ist das Ausnutzen der Rastereinrichtung für Elementverteilungsbilder des SIMS. Mit Hilfe eines Graphikprogramms läßt sich ein Pixelmuster als Vorlage für den Strahlverlauf erstellen. Auf diese Weise ist kein zeitaufwendiges und mit häufigem Abrastern verbundenes Verfahren der Probe nötig. Ist sie optimal positioniert wie in Abbildung 3-7 a), kann mit Hilfe der Rastereinrichtung der Ga-Ionenstrahl entlang des programmierten Musters (vgl. Teilbild b) gelenkt werden. Da die Abrasterung nicht im DC-Modus arbeitet, müssen im Gegensatz zur Methode 1 längere Schneidzeiten in Kauf genommen werden. In der Regel dauert diese Präparation etwa 60 Minuten.

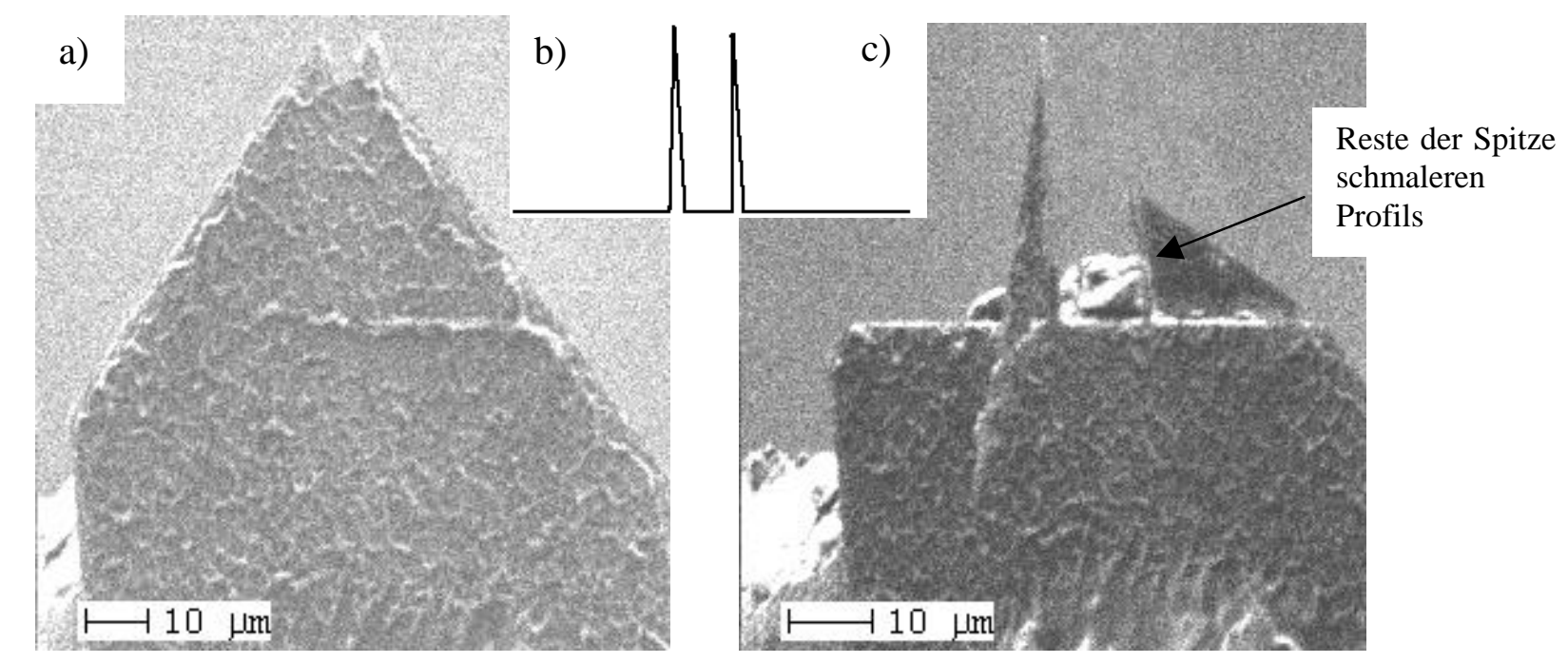

Abbildung 3-7: Schärfung von lithografisch strukturierten TAP-Proben mit dem rastergesteuerten fokussierten Ionenstrahl. a) Ausgangszustand; b) Rastervorlage ; c) nach dem Zusschneiden. Rechts sind noch die Reste des schmaleren rechten Profils zu erkennen.

Eine so nachgeschnittene Spitze zeigt Teilbild c). Als Beispiel für die gute Auflösung sind zwei Spitzen maskiert worden. Beide finden sich nach dem Schneidprozeß wieder.

Eine erfolgreiche Präparation mit ähnlicher Technik mit Hilfe einer Liquid-IonSource wird von LARSON berichtet [LARSON1, LARSON2]. Ein kritischer Punkt ist ebenfalls die starke Ionenmischung in dünneren Randbereichen der Probe. Erst wenn diese Bereiche feldverdampft sind, lassen sich ungestörte Lagenstrukturen erkennen.

\subsection{Elektronenmikroskopie}

Sowohl die für die TAP-Analysen präparierten Spitzen als auch die planaren Schichtpakete wurden im Transmissionselektronenmikroskop (TEM) charakterisiert. 
Für die Untersuchung der Spitzen vor und nach der Beschichtung wurde ein Gerät der Firma Philips, EM $400 \mathrm{~T}$, benutzt. Dieses ist mit einer $\mathrm{LaB}_{6}$-Kathode ausgestattet und wird mit einer Beschleunigungsspannung von $120 \mathrm{kV}$ betrieben.

Die planaren Substrate wurden in einem ersten Schritt querschnittspräpariert und anschließend in der Ionendünnungsanlage der Firma Gatan, Typ Duomill 600, auf eine durchstrahlbare Dicke gedünnt. Näheres zum Verfahren der Querschnittspräparation findet sich in [SCHLEIWIES1]. Diese Querschnittsproben wurden am EM 420 ST der Firma Philips untersucht. In diesem Gerät ist ebenfalls eine $\mathrm{LaB}_{6}-$ Kathode eingesetzt und wird mit $120 \mathrm{kV}$ Beschleunigungsspannung betrieben.

Zusätzlich zum konventionellen Betrieb wurde auch die Hollow-ConeDunkelfeldabbildung (Z-Kontrast, HCDF) zur Erhöhung des Massenkontrastes benutzt. Um diese von der Ordnungszahl $\mathrm{Z}$ abhängigen Bilder zu erhalten, werden zur Abbildung nur unter großem Winkel gestreute Elektronen benutzt. Bei großem Streuwinkel $\Theta \sim 150 \mathrm{mrad}$ überwiegt die inkohärente Streuung am Atomkern gegenüber der kohärenten Streuung am Gitter. Auf diese Weise werden Beugungseffekte weitgehend unterdrückt. Der einfallende Elektronenstrahl rotiert dabei mit einer Frequenz von $0,1 . .1 \mathrm{~Hz}$ auf einem Kegelmantel mit dem Öffnungswinkel $\Theta \approx 80 . .150 \mathrm{mrad}$ um die optische Achse des Mikroskops. Durch Zentrierung einer Objektivblende auf die optische Achse tragen nur Streuanteile aus dem Raumwinkel $\Theta$ zur Abbildung bei.

Die Streuanteile in den Raumwinkel $\Theta$ werden beim längeren Belichten der Fotoplatte aufintegriert, wodurch man eine analysierbare Abbildung erhält. Der Strahlengang ist in Abbildung 3-8 dargestellt. Das räumliche Auflösungsvermögen beträgt bei dieser Methode $\sim 1 \mathrm{~nm}$. Näheres $\mathrm{zu}$ dieser Methode findet sich bei [SCHMITZ2, EWERT].

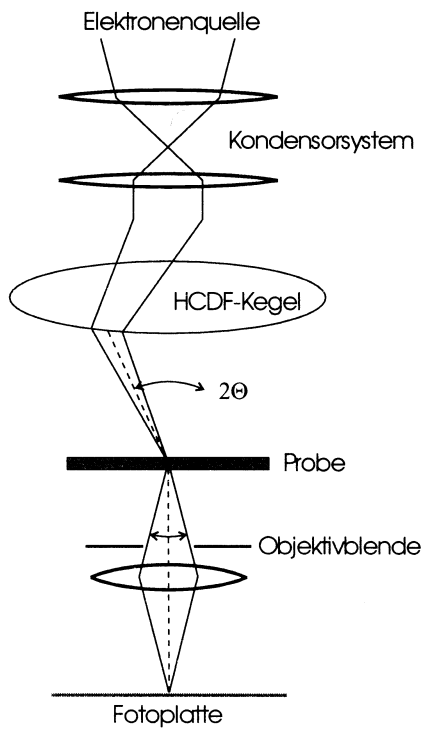

Abbildung 3-8: Schematische Darstellung des Strahlengangs der Hollow-Cone Dunkelfeldabbildung. 


\subsection{Röntgendiffraktometrie}

Die Röntgendiffraktometrie (XRD) wurde als Standardmethode [CuLL] zur Charakterisierung planarer Substrate an zwei unterschiedlichen Geräten angewandt:

a) An dem Vierkreis-Diffraktometer X'Pert der Firma Philips wurden hochauflösende Scans in $\Theta-2 \Theta$ Strahlgeometrie durchgeführt. Die emittierte Röntgenstrahlung hatte eine Wellenlänge von $\lambda=1,7903 \AA(\mathrm{Co}-\mathrm{K} \alpha)$.

Weiterhin wurden mit dem Gerät Texturmessungen [CAHN] durchgeführt, um die laterale kristallographische Kornorientierung der Proben $\mathrm{zu}$ untersuchen. Die Meßergebnisse werden in Polfiguren wiedergegeben.

b) Im Fall der $\mathrm{Al} / \mathrm{Ag}-\mathrm{Schichten}$ ist die Bildung einer neuen Phase Gegenstand der Untersuchung. Mit einem Röntgendiffraktometer der Firma Siemens, Typ D 500, ist es möglich in-situ die Temperatur- und Zeitabhängigkeit der Reaktion zu charakterisieren. Das Gerät ist ebenfalls mit einer Co-Röntgenröhre ausgestattet, mit einer Wellenlänge von $\lambda=1,7903 \AA\left(\mathrm{Co}-\mathrm{K}_{\alpha}\right)$. Die Messungen wurden in $\Theta-2 \Theta$ Strahlgeometrie durchgeführt. Für in-situ Heizmessungen ist die Kammer bis auf einen Enddruck $<10^{-6}$ mbar evakuierbar. Mit Hilfe einer Computersteuerung, die den Heizstrom regelt, läßt sich jeder gewünschte Temperaturverlauf realisieren. Während der Temperaturbehandlung wurden permanent Diffraktogramme aufgezeichnet, wobei ein Scan innerhalb von 10 Minuten aufgenommen wurde. Die maximal nutzbare Temperatur liegt bei $700^{\circ} \mathrm{C}$.

\subsection{Magnetowiderstandsmessungen}

Die Messungen des Magnetowiderstandes der planaren magnetischen Schichtsysteme erfolgte an einem Meßstand in der Bielefelder Arbeitsgruppe von Prof. Reiss [Mrozek]. Als Grundlage dient eine Vier-Punkt-Meßmethode. Längs der Oberfläche der zu vermessenden Probe werden vier in Reihe liegende Kontakte aufgebracht. Die beiden Äußeren leiten einen Konstantstrom durch die Probe während die inneren die Spannung abgreifen. Von besonderer Bedeutung ist die Höhe des Stromes. Er muß so gewählt werden, daß ein genügend hohes RauschSignal-Verhältnis meßbar ist - und genügend klein, um eine Erwärmung der Probe durch den Stromfluß zu verhindern. Bekanntermaßen ist die Höhe des GiantMagnetoResistance-Effektes stark abhängig von der Temperatur. Über ein hochohmiges, hochauflösendes Voltmeter wird der Spannungsabfall ausgelesen und der Widerstand der Probe auf $1 \cdot 10^{-4} \Omega$ genau bestimmt. Der Vorteil dieser Methode liegt in den vernachlässigbar kleinen Widerständen der Kontakte im Vergleich zu dem hochohmigen Meßgerät. Alle Messungen wurden in der CIP-Geometrie durchgeführt, das heißt der Strom floß parallel zu den Schichten.

Die kontaktierte Probe wird so zwischen die Polschuhe eines Elektromagneten eingebaut, daß das erzeugte Magnetfeld parallel zum Probenstrom und somit zu den Schichten orientiert ist. Beim anschließenden computergesteuerten Meßvorgang wird 
der Magnetfeldbereich vom positiven bis negativen Maximalwert durchfahren. Dabei können Magnetfelder bis zu 0,4 Tesla erzeugt werden. Die Stärke des Magnetfeldes wird mit einer Hallsonde gemessen und zusammen mit dem gemessenen Spannungsabfall aufgezeichnet. Unter Berücksichtigung aller Unsicherheiten wie Temperaturschwankungen und Toleranzen der benutzten Geräte, läßt sich die GMRAmplitude auf 0,1\% genau angeben. In Abbildung 3-9 ist beispielhaft eine Magnetowiderstandskurve dargestellt. Auf der linken Ordinate ist das Meßsignal bei konstantem Strom aufgetragen. Dieses kann mit Gleichung(3.11) in den Magnetowiderstand umgerechnet werden, der in Prozent-Einheiten auf der rechten Achse skaliert ist:

$$
\frac{\Delta R}{R_{\text {Min }}}=\frac{R\left(H_{e x t}\right)-R_{\text {Min }}}{R_{\text {Min }}} .
$$

Der maximale $\Delta \mathrm{R} / \mathrm{R}_{\text {Min }}-$ Wert wird als GMR-Effektamplitude $\mathrm{GMR}_{\mathrm{Max}}$ bezeichnet und ist in der Grafik als Punkt A gekennzeichnet:

$$
G M R_{\text {Max }}=\frac{R_{\text {Max }}-R_{\text {Min }}}{R_{\text {Min }}} \cdot 100 .
$$

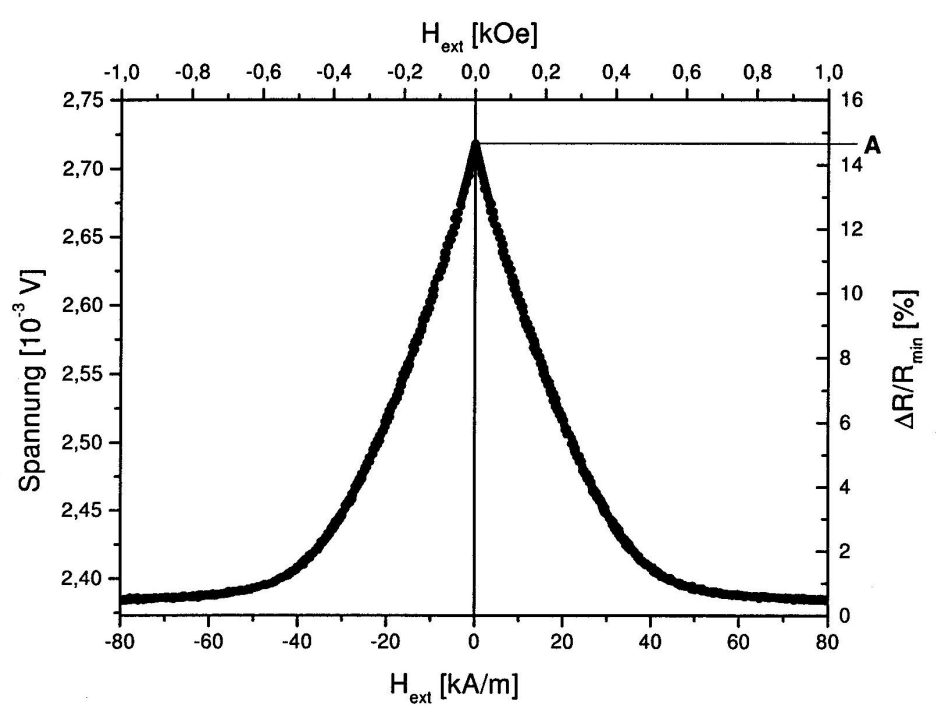

Abbildung 3-9: Typische Meßkurve eines magnetischen Schichtsystems mit GMR-Effekt. Auf der linken Ordinate sind die gemessenen Spannungen und auf der rechten Achse die umgerechneten Magnetowiderstandswerte aufgetragen [HEITMANN1]. 


\section{Nanoanalyse der Interreaktion von $\mathrm{Ag} / \mathrm{Al}$-Schichtpaketen}

\subsection{Vorbemerkungen}

Schichtsysteme bestehend aus Metall/Al-Lagen sind seit vielen Jahren im Brennpunkt aktueller Forschung. Grund dafür ist vor allem die Anwendung des Aluminiums in der Halbleiterindustrie zur Kontaktierung von IC-Schnittstellen oder als Material für Leiterbahnen. Überall da, wo sie in Kontakt mit anderen Komponenten, insbesondere Metallen, stehen, ist die Kenntnis möglicher Phasenbildung durch Interreaktion bei Wärmezufuhr von großer Bedeutung.

Das System Ag-Al ist ein besonders klares Modellsystem zur Aufklärung der kinetischen Abhängigkeiten der Interreaktion.

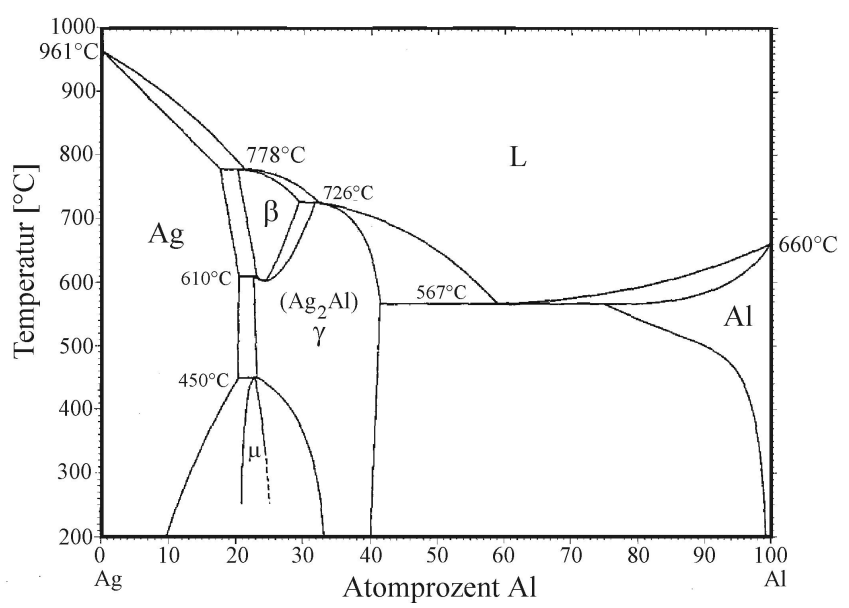

Abbildung 4-1: Phasendiagramm Ag-Al [MASSALSKI]

Beide Komponenten haben fcc-Struktur mit fast identischen Gitterparametern, so daß im Verlaufe der Interreaktion die Spannungskomponente vernachlässigt werden kann. Das Phasendiagramm ist relativ einfach mit drei auftretenden Gleichgewichtsphasen. Vereinfachend kommt hinzu, daß die Hochtemperaturphase $\beta$ zusätzlich noch außerhalb unseres benutzten Temperaturintervalls liegt. Die Bildung der $\mu$-Phase ist kinetisch gehemmt, vermutlich aufgrund der kubisch komplexen Struktur ( $\beta-\mathrm{Mn})$, deren Einheitszelle 20 Atome umfaßt.

Als Bestätigung wird in der Literatur zu Dünnschichtexperimenten am System Ag/Al nur über die beobachtete Bildung der hexagonalen $\gamma$ Phase berichtet [BAGLIN, MARKwITZ]. Da deren Existenzbereich nahe der Stöchiometrie $\mathrm{Ag}_{2} \mathrm{Al}$ liegt, wird eine geordnete Struktur der Gleichgewichtsphase vermutet. Allerdings konnte bis jetzt kein Nachweis einer Fernordnung durch geeignete Beugungsexperimente erbracht werden. Neben diesen Gleichgewichtsphasen ist die Bildung von metastabilen 
GP-Zonen mit fcc Struktur und einer Konzentration zwischen 30 und 60 at.- $\%$ Ag durch Ausscheidung aus übersättigten Ag스-Legierungen bekannt [BAUR2].

In einer frühen Arbeit aus 1968 hat WEAVER die Diffusion und Phasenbildung am Al/Ag-Schichtsystem durch Messung der optischen Reflektivität untersucht [WEAVER]. Für Lagendicken größer als $100 \mathrm{~nm}$ wurde ein parabolisches Wachstumsgesetz der gebildeten Phase gefunden. Die Aktivierungsenergie bestimmten sie $\mathrm{zu} 1,15 \mathrm{eV}$. Interessanterweise nahm die Reaktionsgeschwindigkeit bei Verkleinerung der Lagendicken unter $50 \mathrm{~nm}$ enorm $\mathrm{zu}$, ohne daß sich die Aktivierungsenergie änderte. Die Autoren interpretierten diese Beschleunigung als verstärkten Einfluß der Korngrenzendiffusion und einer anfänglichen hohen Übersättigung an Leerstellen, die katalytisch auf die Diffusion wirkt.

Von BAGLIN durchgeführte RBS-Untersuchungen der Al/Ag-Schichtreaktion bestätigen ein parabolisches Wachstumsgesetz der Produktphase [BAGLIN], während WESTMORELAND ebenfalls ein schnelles Wachsen im Anfangsstadium beschreibt [WeStMORE]. Übereinstimmend zeigen beide Studien in ihren RBS-Spektren der Frühstadien Kurvenverläufe, die einen unscharfen Phasenübergang beschreiben. Als mögliche Ursache kommen sehr raue Grenzflächen oder aber eine bereits stattfindende Interdiffusion der Komponenten in Betracht. Sie äußern die Vermutung, daß hier nicht mehr ein planarer Reaktionsmechanismus vorliegt. Im nächsten Schritt des Experimentes wurde die Größe der Ag-Körner durch Wärmebehandlung variiert. Im Ergebnis zeigt sich eine klare Abhängigkeit der Reaktionsgeschwindigkeit von der Korngröße der Ag-Lage. Je kleiner die Körner, desto höhere Reaktionsraten wurden erreicht, was auf eine starke Abhängigkeit von der Korngrenzendiffusion hinweist. Damit im Einklang steht die erneut bestimmte Aktivierungsenergie von $0,86 \mathrm{eV}$. Der Wert liegt sehr nahe an der Aktivierungsenergie der Korngrenzen-Selbstdiffusion von Ag mit 0,9 eV. Aus den gewonnen Daten wurde ein Reaktionsmechanismus vorgeschlagen, nach dem die Produktphase an der Grenzfläche oder in Tripelpunkten von Korngrenzen bevorzugt innerhalb der $\mathrm{Ag}$-Lage gebildet wird. Nachdem sich eine kontinuierliche $\mathrm{Ag}_{2} \mathrm{Al}-\mathrm{Schich}$ gebildet hat, verläuft ihr weiteres Wachstum parabolisch, wird aber durch Diffusion in ihren Korngrenzen bestimmt.

Die von BAGLIN berechnete Aktivierungsenergie ist deutlich niedriger als der von WEAVER ermittelte Wert von 1,15 eV. Dies mag durchaus an den unterschiedlichen Analysemethoden liegen, macht aber deutlich, daß es schwierig ist, aus den vorhandenen Daten eine Aussage über einen entscheidenden Diffusionsprozeß treffen zu wollen. An dieser Stelle sind genauere Analysen notwendig, die einen schnellen Transport über Korngrenzen nachweisen können.

Zusätzlich wird die Bedeutung der Mikrostruktur durch kaloriemetrische Messungen von RoY untermauert [ROY]. In dem durchgeführten Experiment wurde ein Ag/Al-Bilayer in einer DSC-Apparatur mit einer Heizrate von $20 \mathrm{~K} / \mathrm{min}$ erwärmt. Ähnlich wie bereits an anderen metallischen Schichtsystemen, zum Beispiel Nb/Al, $\mathrm{Ni} / \mathrm{Al}$ oder Ti/Al [MiCHAELSEN2], beobachtet, treten zwei exotherme Peaks auf, obwohl nur die Bildung einer Produktphase beobachtet wird. 
Bei Veränderung der Schichtdicke erscheint der erste Peak im Thermogramm an der selben Stelle, nur die Lage des zweiten ist verändert und dominiert mit wachsender Lagendicke. Das Auftreten der Doppelpeaks wird nach der Arbeit von COFFEY interpretiert als ein in zwei Schritten ablaufender Reaktionsprozeß. Danach kommt es im ersten Schritt zur heterogenen Keimbildung an der Grenzfläche und anschließender Ausbildung zur geschlossenen Keimlage geringer Dicke zwischen den Reaktanden. Der folgende zweite Schritt ist charakterisiert durch Dickenwachstum der Produktphase und demnach abhängig vom Antransport der Komponenten durch das Volumen der Produktlage.

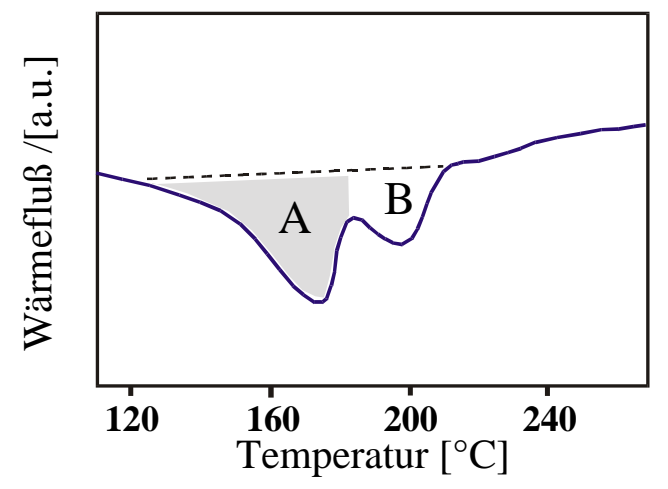

Abbildung 4-2: DSC-Kurve eines $\mathrm{Ag}_{65} \mathrm{~nm} / \mathrm{Al}_{55} \mathrm{~nm}$-Bilayers bei einer Heizrate von $20 \mathrm{~K} / \mathrm{min}$ [ROY].

Eine Anpassung des Modells auf die Meßdaten liefert eine Keimgröße von $50 \mathrm{~nm}$. Dieser Wert liegt im Bereich der Schichtdicke und erscheint physikalisch unvernünftig, da angesichts hoher treibender Kräfte die Keimgröße eher im Bereich von wenigen $\mathrm{nm}$ liegen sollte. Als Mangel ist sicherlich der nicht in das Modell eingehende Einfluß der vorliegenden Mikrostruktur, wie schnelle Diffusionswege durch Korngrenzen zu nennen.

Das Ergebnis geht in Einklang mit allen vorher zitierten Arbeiten. Diese unterstützen den Ansatzpunkt, daß die Mikrostruktur einen dominierenden Einfluß auf den Verlauf der Interreaktion gerade in ihren Frühstadien haben muß. Mit den bisherigen Methoden war allerdings bis jetzt kein experimenteller Nachweis mit chemischer Auflösung auf atomarer Skala möglich. Mit dem Einsatz der Tomographischen Atomsonde sollen diese Mechanismen und Transportwege eingehender auf kleinstmöglicher Skala analysiert werden.

\subsection{Experimentelle Vorarbeiten}

Der Einfluß der Mikrostruktur auf den Verlauf der Interreaktion wurde bereits in der Diplomarbeit von Svenson [Svenson, Schmitz1] am Modellsystem Ag/Al mit den Methoden der Transmissionselektronenmikroskopie (TEM) und des Z-Kontrastes untersucht. In Abbildung 4-3 sind der as-sputtered Zustand und eine Probe nach einer Wärmebehandlung bei $125^{\circ} \mathrm{C}$ für $60 \mathrm{~min}$ dargestellt. Durch die massensensitive Abbildung erscheinen die Al-Schichten wesentlich dunkler als die Ag-reichen Gebiete. Im Ausgangszustand sind chemisch scharfe Grenzflächen zu beobachten. 
Nach einer Auslagerung bei $125^{\circ} \mathrm{C}$ für 60 Minuten zeigen sich deutliche Unterschiede in der Mikrostruktur. Die Z-Kontrast Abbildung 4-3 b) offenbart eine deutliche Eindiffusion von Ag in die Korngrenzen des Al.
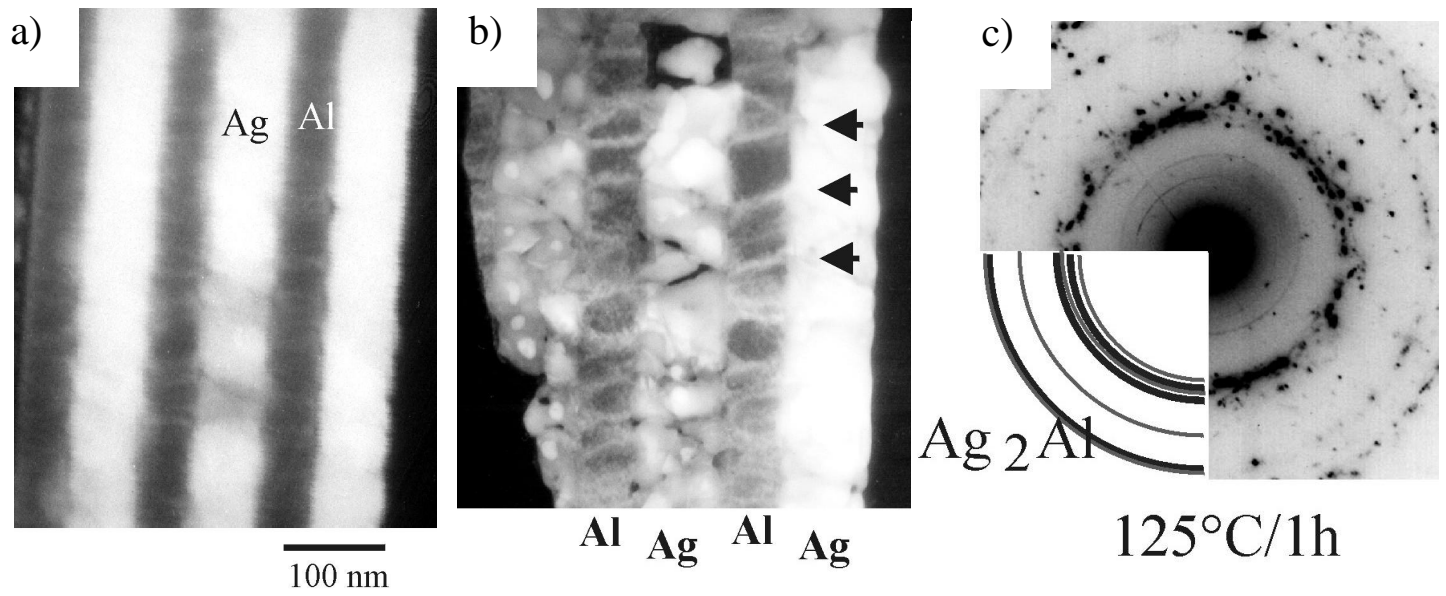

Abbildung 4-3: a) Z-Kontrast Abbildung des Ausgangszustandes. b) Z-Kontrast Aufnahme eines Al/Ag-Schichtpaketes nach Auslagerung bei $125^{\circ} \mathrm{C}$ für $1 \mathrm{~h}$. Die Pfeile markieren deutlich benetzte Al-Korngrenzen. Innerhalb der Ag-Lagen ist ein feines Gefüge erkennbar. c) Die zugehörige Beugungsaufnahme zeigt neben den fcc-Reflexen der reinen Phasen nur die gebildete hexagonale Produktphase [SCHMITZ1, SvENSON].

Über den Vergleich der Helligkeiten läßt sich ein Al-Korngrenzenprofil erstellen, welches einen Ag-Gehalt von mehr als 30 at.-\% demonstriert. Aufgrund der Tatsache, daß bei TEM-Untersuchungen immer Mittelungen über die Probendicke durchgeführt werden und die Korngrenzen nicht parallel zur Strahlachse verlaufen, sollte diese Konzentration als untere Grenze gewertet werden.

Genauere Konzentrationsanalysen der Ag-Schicht sind aufgrund eines ungleichmäßigen Dünnungsprozesses nicht möglich. Es fällt jedoch auf, daß sich innerhalb der Ag-Schicht eine netzartige Struktur ausgebildet hat. Demnach muß eine deutliche Eindiffusion von $\mathrm{Al}$ in die Korngrenzen der Ag-Lage stattgefunden haben. Trotz größerer Dicke der Ag-Lage ist die Korngröße deutlich kleiner als in der Al-Schicht. Der Schwerpunkt der Interreaktion liegt in der Eindiffusion von Al in die Korngrenzen der Ag-Lage.

Bedenkt man die Strukturbreite einer Korngrenze, die etwa bei $1 \mathrm{~nm}$ liegt und vergleicht das mit der Auflösungsgrenze von $1 \mathrm{~nm}$ der Z-Kontrast Abbildung, so ist die Grenze der Methode deutlich. Hier ist eine feinere Analysetechnik gefragt, die Aufschluß über die chemischen Verhältnisse in nanokristallinem Gefüge geben kann. An dieser Stelle setzt die Tomographische Atomsonde an. Sie ermöglicht chemische Analysen auf atomarer Skala. Die mit dieser Methode erzielten Ergebnisse werden im folgenden vorgestellt. 


\subsection{Ergebnisse mit der Tomographischen Atomsonde}

\subsubsection{Eigenschaften der gesputterten Schicht}

Wie in Abschnitt 3.2 beschrieben, wurden Al/Ag-Schichtpakete auf W-Substratspitzen durch Sputterdeposition aufgebracht. Die bei der Herstellung verwendeten Parameter zur Steuerung der Ar-Ionenquelle sind in Tabelle 1 aufgeführt:

\begin{tabular}{|l|c|c|c|c|c|c|c|}
\hline & $\begin{array}{c}\text { Discharge } \\
\text { Voltage } \\
{[\text { [V] }}\end{array}$ & $\begin{array}{c}\text { Current } \\
{[\mathbf{m A}]}\end{array}$ & $\begin{array}{c}\text { Voltage } \\
{[\mathbf{V}]}\end{array}$ & $\begin{array}{c}\text { Accelerator } \\
\text { Voltage } \\
{[\text { [V] }}\end{array}$ & $\begin{array}{c}\text { Dasisdruck } \\
{[\mathrm{mbar}]}\end{array}$ & $\begin{array}{c}\text { Sputterdruck } \\
{[\mathrm{mbar}]}\end{array}$ & $\begin{array}{c}\text { Zeit } \\
{[\mathbf{s}]}\end{array}$ \\
\hline $\begin{array}{l}\text { Reinigung } \\
\text { W-Spitze }\end{array}$ & 45 & 10 & 500 & 200 & $1,310^{-7}$ & $1,910^{-4}$ & 30 \\
\hline $\begin{array}{l}\text { Reinigung } \\
\text { Target }\end{array}$ & 55 & 25 & 600 & 200 & $1,310^{-7}$ & $1,910^{-4}$ & je 120 \\
\hline $\begin{array}{l}\text { Sputtern } \\
\text { Al/Ag- } \\
\text { Bilayer }\end{array}$ & 55 & 12 & 600 & 200 & $1,310^{-7}$ & $1,910^{-4}$ & je 180 \\
\hline
\end{tabular}

Tabelle 1: Sputterparameter zur Ag/Al-Sputterdeposition

Für die Reinigung der W-Spitzen wird der Ar-Ionenstrahl durch Heruntersetzen der Discharge Voltage auf 45 Volt leicht divergent aufgefächert. Während des Sputtervorganges ist ein äußerst paralleler Strahl erwünscht, um ungewünschte Sputtereffekte außerhalb des Targets, wie Substrathalter oder Wasserkühlung zu vermeiden. Dies wird bei 55 Volt Discharge erreicht. Zur sanften Oberflächenreinigung der Spitzen ist insbesondere der Beam Current entscheidend. Er ist auch die entscheidende Größe zur Regulierung der Auftragrate. Große Körner erhält man, durch möglichst langsames Deponieren. Mit einem Beam Current von $12 \mathrm{~mA}$ werden Depositionsraten von 1,4 Ås erzielt. Bei höheren Werten, wie den zur Reinigung der Targets verwendeten hohen Beam Current von $25 \mathrm{~mA}$, steigert sich die Rate auf $10 \AA ̊ \% s$. Entsprechend der Raten fallen auch die Korngrößen der gesputterten Schichten aus. Sowohl die Al-Deckschicht als auch die Ag-Lage sind nanokristallin. Allerdings läßt sich die Korngröße innerhalb der jeweiligen Schicht mit dem Transmissionselektronenmikroskop (TEM) nur schwer bestimmen. Im Gegensatz zu sonst üblichen querschnittspräparierten Proben, die auf wenige Nanometer Dicke gedünnt wurden, überlagern sich die Kornstrukturen des kugelschalen förmigen Schichtpaketes oberhalb und unterhalb des Apex. Zusätzlich ist die aufgebrachte Ag-Lage auf dieser Skala nicht mehr durchstrahlbar. Abbildung 4-4 zeigt eine TEM-Aufnahme einer mit niedriger Rate von 1,4 Ås beschichteten Spitze. Die Kontur der unterliegenden W-Spitze ist nur noch schwach erkennbar. Die Schichtdicke der Ag-Schicht läßt sich aufgrund des schlechten Kontrastes nur schwer auf ungefähr $30 \mathrm{~nm}$ abschätzen. Deutlich hervor tritt die Kornstruktur der oberen Al-Lage. Am Apex der Spitze ist ein Al-Korn mit einer lateralen Größe von etwa $15 \mathrm{~nm}$ erkennbar. 


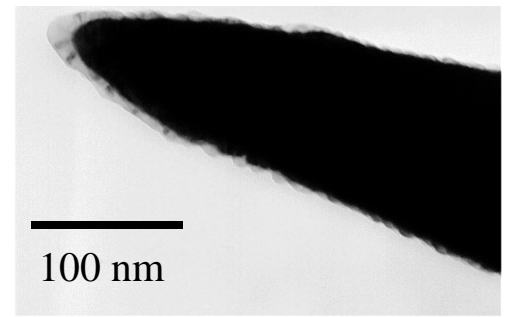

Abbildung 4-4: TEM-Aufnahme eines gesputterten Al/Ag-Bilayers auf eine vorentwickelte W-Spitze. Die Ag-Lage ist aufgrund der geringen Durchstrahlung kaum sichtbar. Der Spitzenradius beträgt etwa $40 \mathrm{~nm}$.

Die aufgebrachte Al-Schicht hat eine Dicke von $30 \mathrm{~nm}$. Bei der Herstellung aller Proben wurden die Raten mit einem Schwingquarz kontrolliert. Es wurde darauf geachtet, daß auf allen Trägerspitzen Schichtpakete gleicher Dicke deponiert wurden. Trotzdem fielen die produzierten Schichten unterschiedlich dick aus. Im Mittel wurden Bilayer mit einer Einzelschichtdicke zwischen 20 und $30 \mathrm{~nm}$ deponiert. Die erreichte Lagendicke ist offensichtlich stark abhängig von der vorher präparierten Form der Trägerspitze und variiert mit dem Spitzenradius und dem Schaftwinkel.

Einen weitere Möglichkeit der Schichtcharakterisierung, die Aufschluß geben würde über die Nanostruktur, wie Korngröße, Wachstumsrichtung, ist die Untersuchung im Feldionenmikroskop. Leider ist aber die Feldverdampfungsfeldstärke von nanokristallinem $\mathrm{Al}$ derart niedrig, daß nur grobe Strukturen durch das an Dekorationslinien bereits kondensierende Ne-Bildgas zu bemerken sind. Erhöht man die Grundspannung $\mathrm{U}_{\mathrm{DC}}$ oder die an der Spitze anliegende Temperatur um eine saubere Abbildung zu erhalten, wird bereits die Schwelle zur Feldevaporation überschritten, was eine Abbildung unmöglich macht.
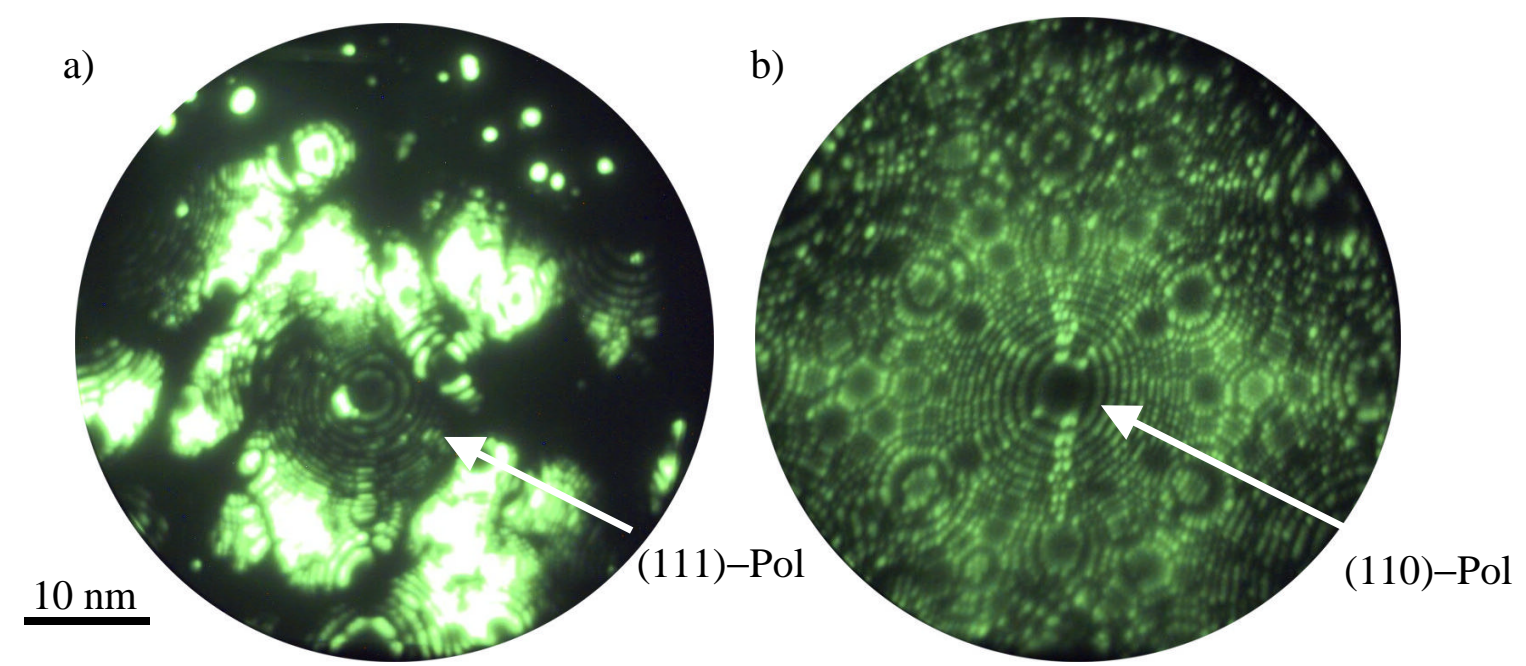

Abbildung 4-5: Feldionenmikroskopische Abbildung der a) gesputterten Ag-Schicht. Zu erkennen sind durch den (111)-Pol laufende Schraubenversetzung und verschiedene Korngrenzen. Die mittlere Korngröße liegt zwischen 5 und $10 \mathrm{~nm}$. b) Abbildung der unterliegenden W-Substratspitze. Die Ag-Schicht ist mit ihrer (111)-Wachstumsrichtung direkt auf dem (110)-Pol der W-Spitze aufgewachsen. 
Lediglich die Ag-Schicht läßt sich mit einem Neon-Partialdruck von $5 \cdot 10^{-5}$ mbar sauber abbilden. Ein solches feldionenmikroskopisches Bild einer bei $115^{\circ} \mathrm{C}$ für 30 Minuten wärmebehandelten Probe ist in Abbildung 4-5 a) dargestellt.

Zentral ist ein (111)-Pol mit einer Schraubenversetzung zu sehen. Dies bestätigt die Aufwachsrichtung von fcc-Ag, mit deren Netzebenen die Tiefeneichung kontrolliert wird. Bis auf das große zentrale Korn sind viele einzelne Ag-Körner mit einer Größe zwischen 5 und $10 \mathrm{~nm}$ erkennbar. Sie sind voneinander sowohl durch Kleinwinkelals auch durch Großwinkel-Korngrenzen getrennt. Aufgrund der geringen Krümmung des (111)-Pols mußte die Grundspannung für dessen Abbildung so weit erhöht werden, das andere stärker gekrümmte Bereiche überstrahlt erscheinen.

Im rechten Teil von Abbildung 4-5 ist die Oberfläche der unterliegenden W-Substratspitze zu sehen. Sie ist einkristallin mit (110)-Drahttextur. Vergleicht man beide Abbildungen, so fällt auf, daß beide Hauptpole, der Ag (111)- und der W(110)-Pol, übereinander liegen. Anhand von Rekonstruktionen gemessener Volumina läßt sich auch für die Al-Schicht feststellen, daß deren (111)-Pol genau auf dem Ag-Hauptpol aufgewachsen ist. Die Schichten wachsen also wohlorientiert mit ihrem Hauptpol entlang der Drahtachse auf.

Die gesputterten Proben wurden in einem UHV-Ofen wärmebehandelt und anschließend in der Tomographischen Atomsonde charakterisiert. Die in Tabelle 2 aufgeführten Parameter wurden dabei für die TAP-Untersuchungen verwendet.

\begin{tabular}{|l|l|}
\hline \multicolumn{2}{|l|}{ Meßparameter TAP } \\
\hline Pulsverhältnis $\alpha=\mathbf{U}_{\mathbf{P}} /\left(\mathbf{U}_{\mathbf{P}}+\mathbf{U}_{\mathbf{D C}}\right)$ & $17 \%$ \\
\hline Pulsfrequenz & $2000 \mathrm{~Hz}$ \\
\hline Detektionsrate & $\approx 0,008$ Ionen / Puls \\
\hline Detektionseffizienz & 0,5 \\
\hline Temperatur Kühlkopf & $30 \mathrm{~K}$ \\
\hline \hline Feldverdampfungsfeldstärke $\boldsymbol{A l}^{4}$ & $19 \mathrm{~V} / \mathrm{nm}$ \\
\hline Feldverdampfungsfeldstärke $\mathbf{A g}^{4}$ & $24 \mathrm{~V} / \mathrm{nm}$ \\
\hline
\end{tabular}

Tabelle 2: Meßparameter der Tomographischen Atomsonde

Haben die beteiligten Komponenten unterschiedliche Feldverdampfungsfeldstärken wie im System Ag-Al, so kann die Konzentrationsbestimmung durch bevorzugtes Feldverdampfen eines Elementes verfälscht werden. Präferentielles Verdampfen läßt sich durch eine möglichst tiefe Kühlung der Probe oder durch ein großes Pulsverhältnis unterdrücken [MILLER]. Beides ist jedoch für die mechanische Stabilität dünner Schichten auf einem gekrümmten Substrat ungünstig. Wie bereits erwähnt, ist

\footnotetext{
${ }^{3}$ Durch thermische Verluste beim Ankoppeln des Probenhalters über Kupferlitzen an den Kühlkopf liegt die eigentliche Temperatur an der Spitze etwa 10 - $15 \mathrm{~K}$ höher.

${ }^{4}$ vgl. [MILLER]
} 
ein schonender Meßverlauf für die Haftung der Schichten auf dem Substrat notwendig. Deswegen wurde die Temperatur der Probe mit $\mathrm{T}_{\mathrm{KK}}=30 \mathrm{~K}$ leicht über der erreichbaren tiefsten Temperatur von $15 \mathrm{~K}$ gewählt. Inwieweit bei diesen schonenderen Bedingungen eine zuverlässige Analyse möglich ist, wurde durch Eichmessungen festgestellt. Dazu wurde eine Eichlegierung mit 8,5 at.-\% Ag erschmolzen und TAP-Analysen bei verschiedenen Temperaturen durchgeführt.

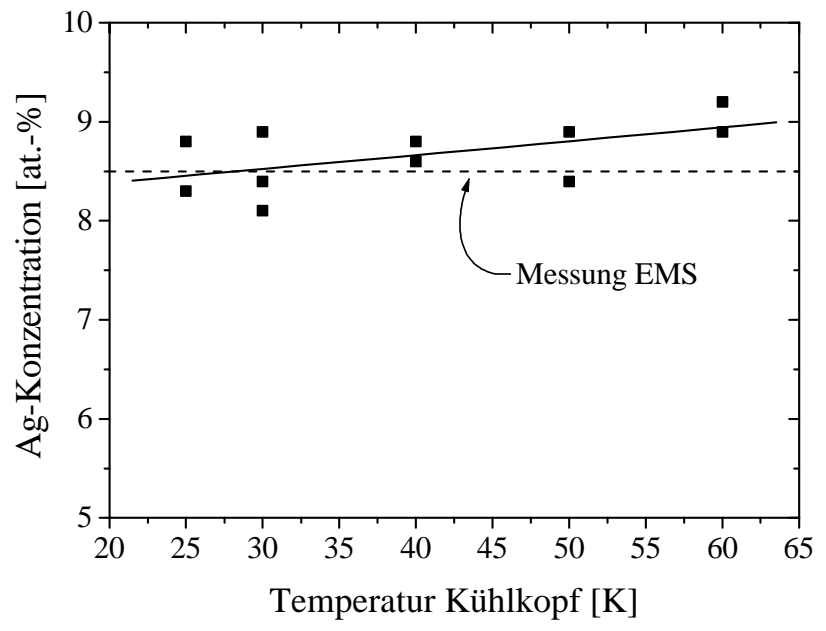

Abbildung 4-6: Eichmessung zur Bestimmung der Temperaturabhängigkeit der gemessenen Ag-Konzentration

Wie man aus der Auftragung der gemessenen Ag-Konzentration über die Temperatur des Kühlkopfes in Abbildung 4-6 entnimmt, ist die Abweichung von der realen Konzentration bei einer Temperatur von $30 \mathrm{~K}$ ausreichend klein. Generell ist das bevorzugte Verdampfen von Al bei erhöhter Temperatur eher gering, wie die Gerade zur Visualisierung der Tendenz deutlich macht. Die gemessenen Konzentrationen, insbesondere die der wärmebehandelten Spitzen, sind also bezüglich einer selektiven Feldverdampfung mit einem sehr geringen Fehler behaftet, der innerhalb der statistischen Streuung der Daten liegt.

\subsubsection{Experimentelle Ergebnisse der Tomographischen Atomsonde}

As-sputtered $/ 9^{\circ} \mathrm{C}$ für $11 \mathrm{~min}$. In Abbildung 4-7 ist die TAP-Analyse eines $\mathrm{Al} / \mathrm{Ag}-\mathrm{W}$ Schichtpaketes im Ausgangszustand dargestellt. Die einzelnen Atomsorten sind farblich markiert: Al-Atome gelb, Ag-Atome rot. Zusätzlich ist noch die W-Trägerspitze, blau markierte Atome, erkennbar. Der Maßstab der Rekonstruktion ist durch einen $1 \mathrm{~nm}^{3}$ Würfel angegeben. Die Grenzfläche zwischen Al- und Ag-Schicht scheint sehr eben auszufallen. Durch Einfügen einer Isokonzentrationsfläche läßt sich der Verlauf der Grenzfläche genau festlegen. Dazu senkrecht wird der Analysezylinder zur Volumenauswahl justiert, um eine möglichst hohe Meßschärfe in einem Konzentrationsprofil zu garantieren. In Teilbild b) ist ein solches Profil wiedergegeben. Es zeigt einen scharfen Übergang von $10 \mathrm{zu}$ 90 at.-\% Al innerhalb von $1 \mathrm{~nm}$. Diese Breite des Übergangs liegt über der Grenze 
der Tiefenauflösung der Methode von 0,2 nm. Da aber selbst bei entmischenden Systemen wie $\mathrm{Cu} / \mathrm{Co}$ eine Grenzflächenschärfe von etwa $1 \mathrm{~nm}$ gemessen wurde, kann eine bereits einsetzende Schichtreaktion ausgeschlossen werden. Daher ist die breite Übergangszone mit Sicherheit auf den Herstellungsprozeß zurückzuführen. Jedoch ist sie nicht verursacht durch den Prozeß des Ionenmischens beim Depositionsproze $\beta$. Im weiteren Kontext wird ausgeführt, daß das Ionenmischen lediglich innerhalb einer Tiefe von $3 \AA$ stattfindet. Auch das manuelle Umschalten der Targets innerhalb von 2 Sekunden führt bei der langsamen Auftragrate von $1,4 \AA$ \/s zu einer maximalen Durchmischung von $3 \AA$. Es müssen also noch andere Gründe existieren, die für diese Durchmischungszone verantwortlich sind.
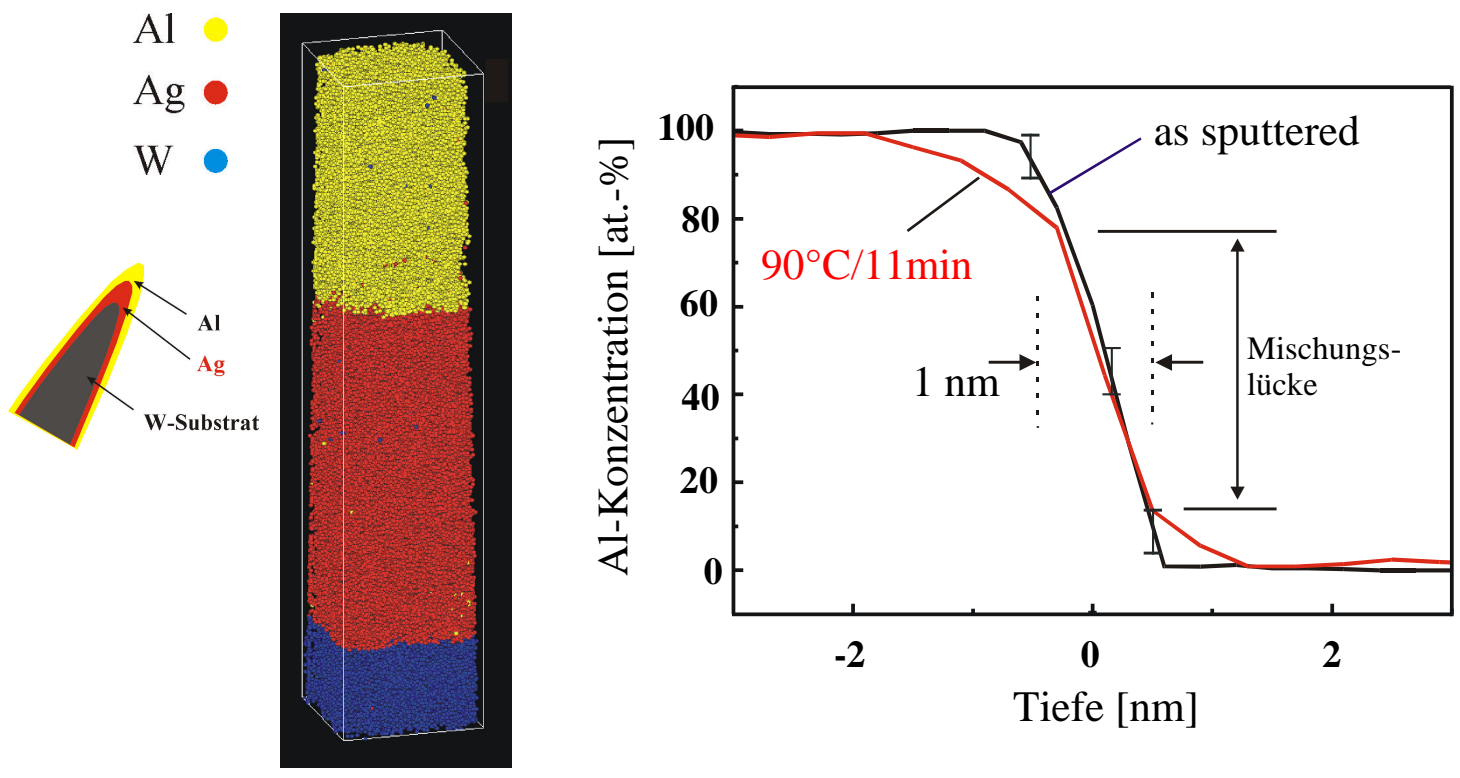

Abbildung 4-7: a) 3D-Rekonstruktion des Al/Ag-Schichtpaketes im Ausgangszustand. b) Konzentrationsprofil entlang der Meßachse der Probe im Ausgangszustand und nach einer Wärmebehandlung bei $90^{\circ} \mathrm{C}$ für 11 Minuten.

Nach einer kurzen Auslagerung einer ähnlichen Probe bei $90^{\circ} \mathrm{C}$ für 11 Minuten zeigt die Rekonstruktion des Meßvolumens keine merkliche Änderung. Beim Vergleich der Konzentrationsprofile zeigen sich jedoch signifikante Abweichungen, die Dank der hohen Auflösung der Tomographischen Atomsonde registriert werden können. Auf beiden Seiten der Grenzfläche haben sich Interdiffusionszonen mit unterschiedlicher Ausdehnung gebildet. Auf der Al-Seite ist die Zone $2 \mathrm{~nm}$ ausgedehnt, in der Ag-Schicht dagegen nur $1 \mathrm{~nm}$. Um Aufschluß über den Diffusionsmechanismus zu erhalten, vergleichen wir diese Weiten mit denen durch Volumendiffusion erreichbaren Werten. Die auf die verwendete Auslagerungstemperatur extrapolierten Diffusionsdaten sagen eine Eindiffusion von $\mathrm{Ag}$ in die Al-Schicht von fast $7 \AA$ vorher, wohingegen sich im $\mathrm{Ag}$ eine nicht mehr analysierbare Diffusionszone von $0,006 \AA$ ausbilden sollte. Die Ausbildung dieser Interdiffusionszonen läßt sich daher nicht alleine durch den Prozeß der Volumendiffusion erklären. 2D-Konzentrationsschnitte, die die lokale Konzentration durch Falschfarben markieren, zeigen jedoch keine Anzeichen für einen 
unterstützenden Einfluß von Korngrenzen-Diffusion auf größerer Skala. Allerdings ist nicht auszuschließen, daß schon Korngrenzenbenetzung auf einem sehr kleinen Maßstab stattfindet.

Eine weitere auf den Prozeß beschleunigend wirkende Ursache, ist eine Übersättigung an Leerstellen. Dies wird üblicherweise auf die Herstellungsmethode, den Sputterprozeß zurückgeführt. Das Vorhandensein einer solchen Leerstellenaglomeration läßt sich mit einer Monte-Carlo-Simulation, durchgeführt mit dem Programm SRIM-2000, überprüfen. Es wird im Anhang näher vorgestellt und eine erweiterte Übersicht der Ergebnisse gegeben. Fazit ist aber, daß die beim Beschichtungsproze $\beta$ abgesputterten Al-Targetatome eine maximale TeilchenEnergie von $14 \mathrm{eV}$ besitzen. Diese ermöglicht eine maximale Eindringtiefe von $3 \AA$ und ist damit nicht korrelierbar mit der Breite der Interdiffusionszone. Außerdem ist die Anzahl der durch den Beschuß zusätzlich gebildeten Leerstellen vernachlässigbar gering. Diese Betrachtung macht jedoch keine Aussage über die Leerstellenbildung durch unvollständiges Schichtwachstum beim Depositionsprozeß. Ein Einfluß von Leerstellenübersättigung auf die Interdiffusion kann demnach ebenso wenig ausgeschlossen werden, wie eine geringe Korngrenzenbenetzung.

Vergleicht man beide Konzentrationsprofile, so offenbart sich zusätzlich noch die Existenz einer Mischungslücke. Diese erstreckt sich von 80 at.-\% Al bis zu 10 at.$\% \mathrm{Al}$. Interessanterweise tritt bei der Entmischung übersättigter Al-Legierungen unter Bildung von Guinier-Preston-Zonen mit fcc-Struktur eine übereinstimmende metastabile Mischungslücke auf [BAUR1, OsamURA]. Vergleicht man die gefundenen Löslichkeiten mit denen des Gleichgewichtsphasendiagramms, so findet man erstaunlicherweise eine starke Übersättigung auf der Al-Seite mit 20 at.- $\% \mathrm{Ag}$ gegenüber einer Gleichgewichtslöslichkeit von 1 at.-\% Ag. Bis zu diesem Punkt verläuft die Reaktion der Schichten konform mit den in der Literatur eingängigen Modellen der Interreaktion planarer Schichten [HeuRLE1]. In einem ersten Schritt erfolgt die Übersättigung durch Interdiffusion. Diese führt zu einer treibenden Kraft für die anschließende Keimbildung der Produktphase.

Zur Erweiterung des physikalischen Verständnisses des Reaktionsverlaufes sollten weitere Auslagerungen isotherm bei verschiedenen Zeiten geschehen. Allerdings hat sich gezeigt, daß die Zeitskala der verschiedenen Reaktionsschritte derart unterschiedlich ist, daß nicht alle Stadien bei einer Temperatur durchlaufen werden können. Deswegen wurden steigende Temperaturen gewählt, bis die Analyse ein Ende des Reaktionsverlaufes zeigte.

$100^{\circ} \mathrm{C}$ für $15 \mathrm{~min} / 115^{\circ} \mathrm{C}$ für $30 \mathrm{~min}$. Überraschenderweise verläßt die Reaktion den modellhaften Verlauf planarer Schichtsysteme. Dies wird deutlich nach einer Auslagerung der Probe bei $100^{\circ} \mathrm{C}$ für 15 Minuten, dargestellt in der Rekonstruktion in Abbildung 4-8. Das rekonstruierte Volumen zeigt keinen planaren Reaktionsverlauf, sondern deutlich die Diffusion des $\mathrm{Al}$ in die Korngrenzen der $\mathrm{Ag}-$ Schicht. Die so umschlossenen $\mathrm{Ag}-\mathrm{Körner}$ haben einen Durchmesser von 5 bis $10 \mathrm{~nm}$, der aus Konzentrationsquerschnitten und Isokonzentrationsflächen bestimmt werden kann. In den dargestellten Querschnitten sind die jeweiligen Konzentrationen durch Falschfarben kodiert. Die Skala reicht dabei von blau für reines Ag bis zur 
roten Markierung von 60 at.-\% Al. Neben dem deutlichen Korngrenzennetzwerk fällt die Existenz eines Korngrenzen-Tripelpunktes mit sehr hohem Al-Gehalt auf. Nahe dieses Punktes wurde durch Anpassen eines Analysezylinders senkrecht zu berechneten Isokonzentrationsflächen der Al-Gehalt dieser Diffusionszonen und deren Breite sehr genau bestimmt.
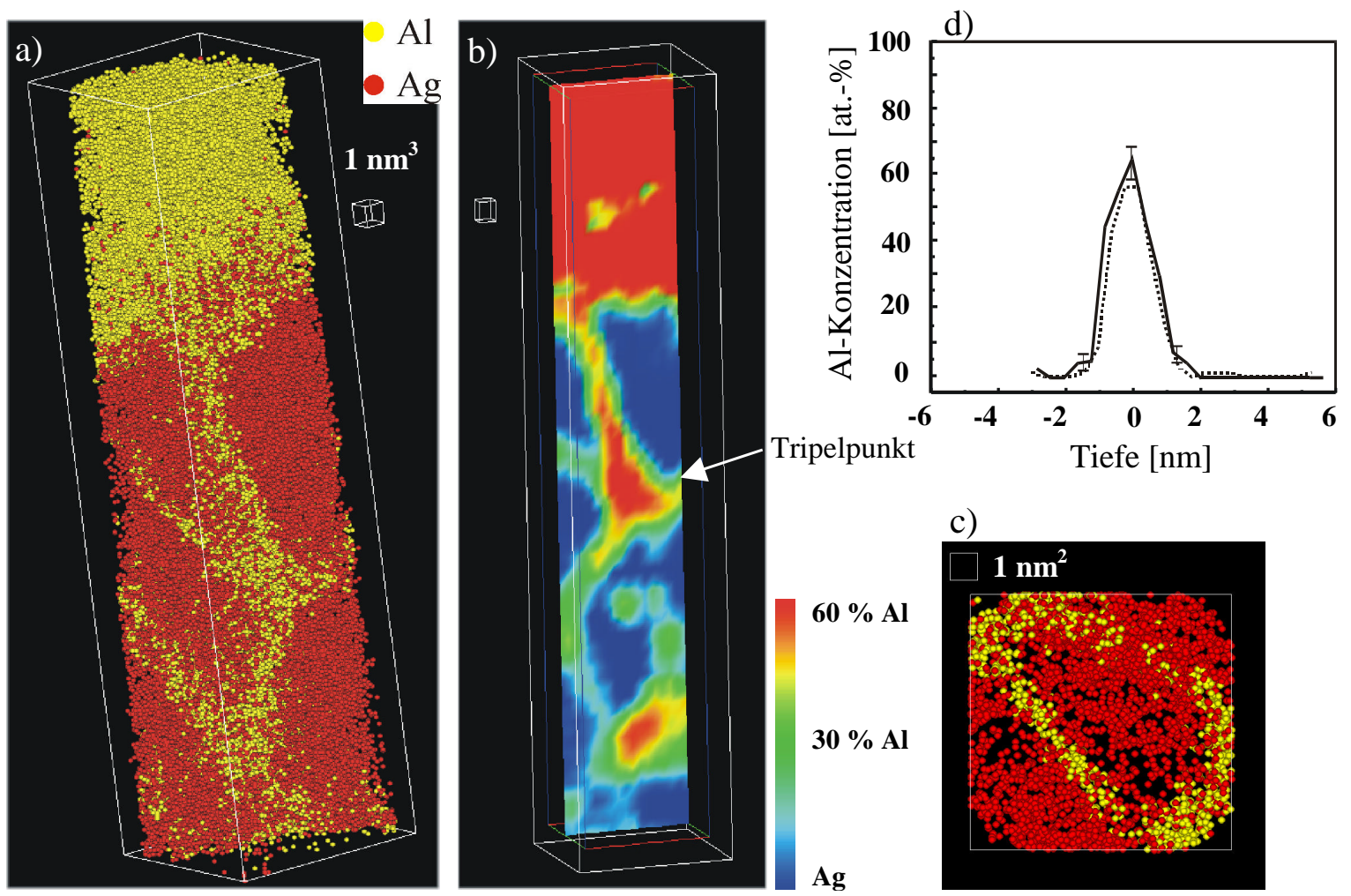

Abbildung 4-8: a) 3D-Rekonstruktion einer getemperten Probe $\left(100^{\circ} \mathrm{C} / 15 \mathrm{Min}\right)$. Deutlich tritt die Eindiffusion des Al in die Korngrenzen der Ag-Schicht hervor. b) Tomographischer Schnitt in der Mitte des Meßvolumens parallel zur Analyserichtung. Die vorliegenden Konzentrationen sind durch Falschfarben markiert. c) Ebener Schnitt einer Dicke von 1 nm durch die Atomverteilung senkrecht zur Achse. Die Größe des Ag-Korns liegt bei etwa 5 nm. Zu erkennen sind Al-Anreicherungszonen an den Schnittstellen der Korngrenzen. d) Konzentrationsprofile senkrecht durch Korngrenze nahe des Tripelpunktes.

Dabei zeigt sich eine sehr hohe Al-Konzentration von bis zu 60 at.-\%. Diese übertrifft den Al-Gehalt der erwarteten $\gamma$-Gleichgewichtsphase um das Doppelte. Die Breite dieser Segregationszone wird in Konzentrationsprofilen $\mathrm{zu} 2 \mathrm{~nm}$ bestimmt. Bei weiteren Auslagerungsschritten verbreitert sich diese Zone. Nach einer Wärmebehandlung für 30 Minuten bei $115^{\circ} \mathrm{C}$ bleiben nur noch einige der größten Ag-Körner erhalten. Die Al-reiche Schale wächst offensichtlich stetig in Richtung Zentrum der $\mathrm{Ag}-$ Körner, während die Grenzfläche zwischen $\mathrm{Ag}-$ Korn und wachsender Korngrenzenphase scharf bleibt (vgl. Abbildung 4-9 c). 

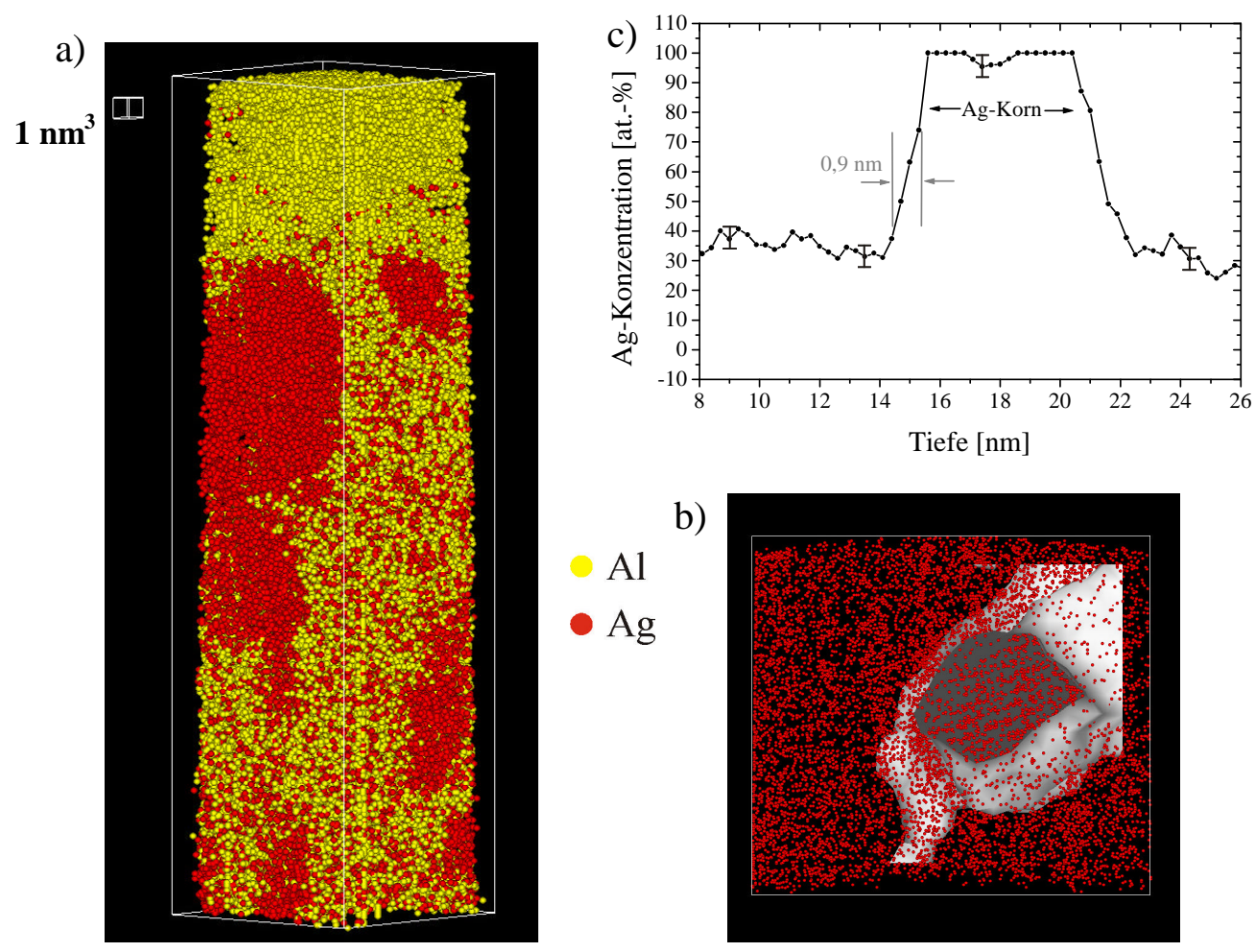

Abbildung 4-9: a) 3D-Rekonstruktion eines bei $115^{\circ} \mathrm{C} / 30$ Min ausgelagerten Al/Ag-Schichtpaketes. Nur noch wenige Ag-Körner sind übrig geblieben. Dazwischen hat sich eine breite Zone der Zusammensetzung $\mathrm{Al}_{2} \mathrm{Ag}$ gebildet. b) Ausschnittvergrößerung eines Ag-Korns umgeben von einer Isokonzentrationsfläche von 90 at.-\% Ag. Die aufgelösten Netzebenen zeigen einen scharfen Abbruch in die umgebende Matrix. c) Konzentrationsprofil senkrecht durch das Ag-Korn.

Die Konzentrationsanalyse senkrecht zur Oberfläche eines Ag-Korns (vgl. Abbildung 4-9 c) zeigt einen sehr großen Konzentrationsgradienten ähnlich dem der as-sputtered Probe mit einer Übergangsbreite von nur 0,9 nm. Das führt zu dem Schluß, daß es sich bei dieser Grenzfläche um eine echte Phasengrenze handeln muß. Zusätzlich gibt die Rekonstruktion des Probenvolumens Hinweise darauf, daß diese Phasengrenze inkohärenten Charakter hat. Dies soll mit einem Ausschnitt in Teilbild b) verdeutlicht werden. Innerhalb des Ag-Korns lassen sich die Netzebenen eindeutig auflösen. Sie werden allerdings an einer scharfen Grenze, die zur Verdeutlichung durch eine Isokonzentrationsfläche dargestellt wurde, nicht in die angrenzende Phase fortgesetzt.

$140^{\circ} \mathrm{C}$ für $30 \mathrm{~min}$. Abbildung 4-10 zeigt einen tomographischen Schnitt durch das Meßvolumen eines $\mathrm{Al} / \mathrm{Ag}$-Schichtpaketes nach einer Wärmebehandlung bei $140^{\circ} \mathrm{C}$ für 30 Minuten. Die zur Al-Schicht benachbarten Ag-Körner sind vollkommen aufgezehrt. Durch diese Messung wurde senkrecht zur Analyserichtung ein Konzentrationsschnitt gelegt, mit der Farbskala von rot-100 at.- $\%$ Al zu blau-100 at.$\%$ Ag (vgl. Abbildung 4-10). Neben den Resten der reinen Ag- und Al-Lage ist deutlich eine ausgedehnte Al-reiche Zwischenlage ausgebildet. Sie erstreckt sich über eine Dicke von fast $40 \mathrm{~nm}$. Mit Hilfe der Konzentrationsanalyse bestimmt sich ihre Zusammensetzung zu konstanten 67 at.-\% Al, was der Stöchiometrie einer 
$\mathrm{Al}_{2} \mathrm{Ag}-\mathrm{Phase}$ entspricht. Deren Existenz wird allerdings nicht vom Gleichgewichtsphasendiagramm vorhergesagt. Trotzdem muß diese Phase als signifikant für die Frühstadien der Interreaktion akzeptiert werden, da sie in 8 weiteren, unabhängigen Messungen reproduziert werden konnte. Sehr deutlich ist auch der konstante Verlauf ohne jeglichen Konzentrationsgradienten innerhalb der gebildeten Phase und scharfen Phasengrenzen zu den Ausgangsschichten. Der Übergangsbereich zwischen intermediärer Phase und Al-Schicht liegt mit einer Schärfe von unter $1 \mathrm{~nm}$ wieder vergleichbar mit der as-sputtered Probe. Auf der $\mathrm{Ag}-$ Seite läßt sich in dem Konzentrationsprofil keine derartige Schärfe messen, da innerhalb des ausgesuchten Zylindervolumens eine Al-benetzte Korngrenze verläuft.
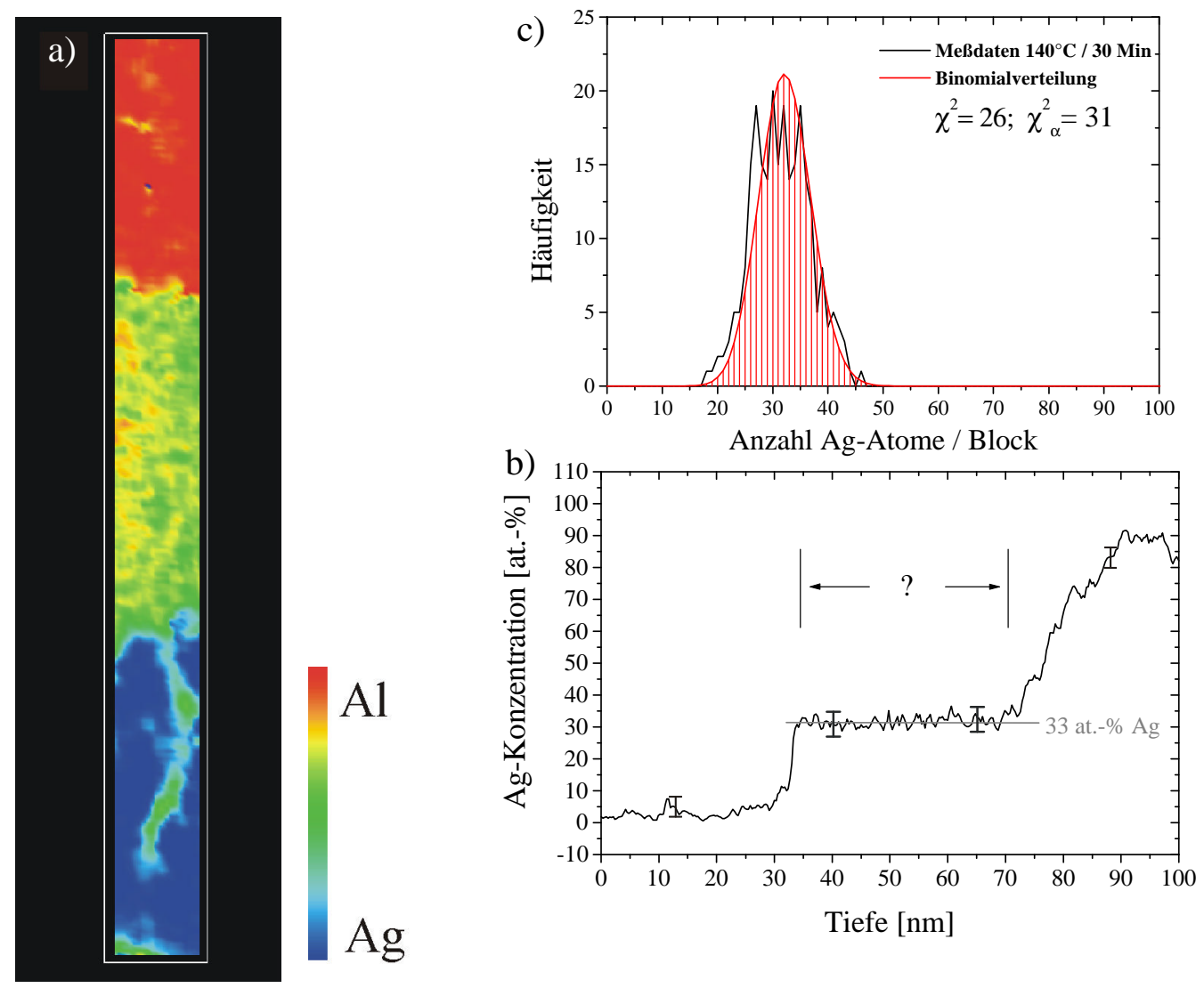

Abbildung 4-10: a) Tomographischer Schnitt durch das aufgenommene Meßvolumen und b) das zugehörige Konzentrationsprofil parallel zur Analyserichtung. c) $\chi^{2}$-Test innerhalb der intermediären Produktphase.

Zur Überprüfung der Homogenität der gebildeten $\mathrm{Al}$-reichen Phase wurde ein $\chi^{2}-$ Test (vgl. Abschnitt 3.1.3) durchgeführt. Dabei wird stichprobenartig das gesamte Meßvolumen in kleinen Würfeln abgerastert und eine Häufigkeitsverteilung der in jeder Stichprobe von 100 Atomen enthaltenen Ag-Atome erstellt. Diese Verteilung wird mit einer für eine homogene Durchmischung erwarteten Binomialverteilung verglichen. Der Schwerpunkt der Verteilung liegt dabei immer bei der mittleren Konzentration des gesamten Testvolumens. Eine Aussage über die Signifikanz der 
Abweichung, das heißt, ob die Ag-Atome homogen oder inhomogen verteilt sind, erhält durch Vergleich des berechneten $\chi^{2}$-Wertes mit einem kritischen Wert $\chi_{\alpha}{ }^{2}$, der für ein Wahrscheinlichkeitsniveau von $95 \%$ steht.

Das Ergebnis des $\chi^{2}$-Test innerhalb der intermediären Schicht ist in Abbildung 4-10 c) dargestellt. Der berechnete Wert $\chi^{2}=26$ liegt deutlich unterhalb des kritischen Wertes $\chi_{\alpha}{ }^{2}=31$. Die gebildete Phase mit einer Konzentration von 67 at.-\% $\mathrm{Al}$ ist demnach in ihrem Existenzbereich eher homogen.

$200^{\circ} \mathrm{C}$ für $\quad 3^{\circ} \mathrm{min} / 250^{\circ} \mathrm{C}$ für $30 \mathrm{~min}$. Bei weiterer Steigerung der Auslagerungstemperatur auf $200^{\circ} \mathrm{C}$ ist sowohl die $\mathrm{Al}-$ als auch die Ag-Schicht
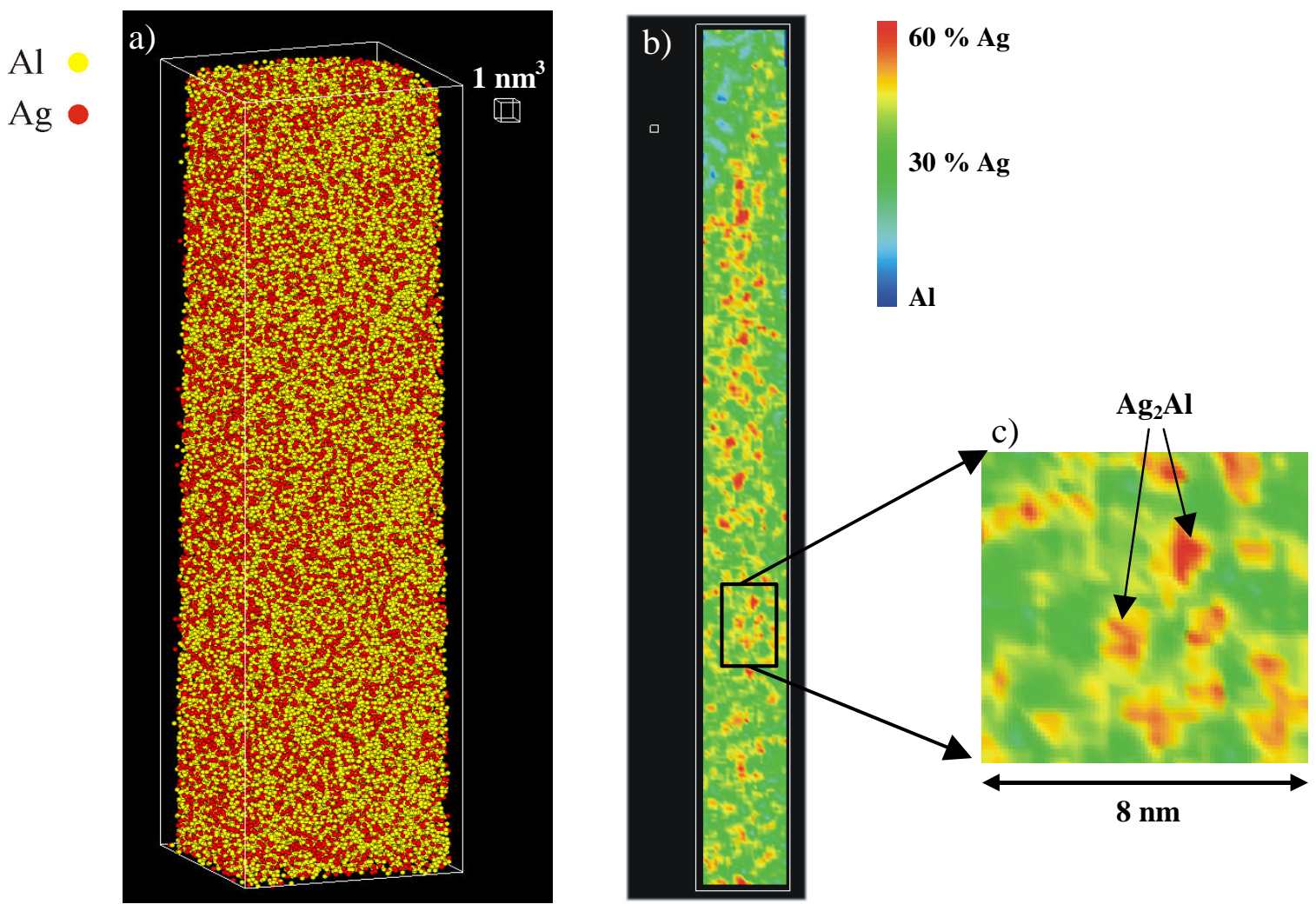

Abbildung 4-11:a) 3D-Rekonstruktion eines bei $250^{\circ} \mathrm{C}$ für 30 Minuten ausgelagerten Al/Ag-Schichtpaketes. b) Tomographischer Schnitt durch das Messvolumen. c) Ausschnittsvergrößerung zur Demonstration von $\mathrm{Ag}_{2} \mathrm{Al}$-Anreicherungen.

vollkommen aufgezehrt. Allerdings offenbart ein $\chi^{2}-$ Test, daß das Testvolumen keineswegs homogenen Charakter besitzt. In Abbildung 4-12 a) zeigt der Verlauf der Ag-Verteilung deutliche Abweichungen in den Randverläufen von der Binomialverteilung. Ein Vergleich von $\chi^{2}=538$ mit $\chi_{\alpha}{ }^{2}=43$ beschreibt eine deutliche Inhomogenität der Probe.

Das rekonstruierte Volumen der bei $250^{\circ} \mathrm{C}$ getemperten Probe (vgl. Abbildung 4-11 a) demonstriert augenscheinlich keine Inhomogenität. Ein Konzentrationsschnitt durch das Volumen in Teilbild b) zeigt allerdings eine netzartige Struktur. Die 
farbliche Kodierung reicht von blau: 100 at.- $\%$ Al zu rot: 60 at.- $\%$ Ag, wodurch die erwartete $\mathrm{Ag}_{2} \mathrm{Al}-$ Phase markiert wird.

a)

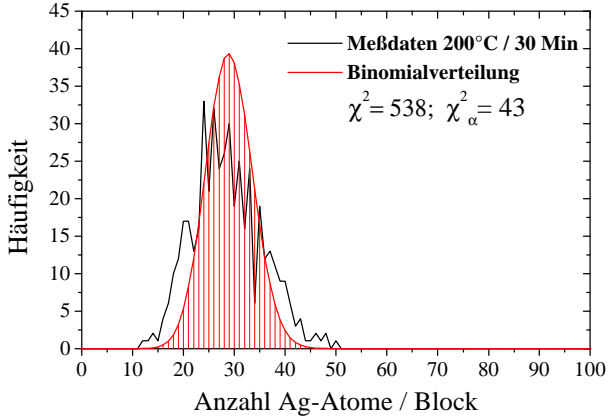

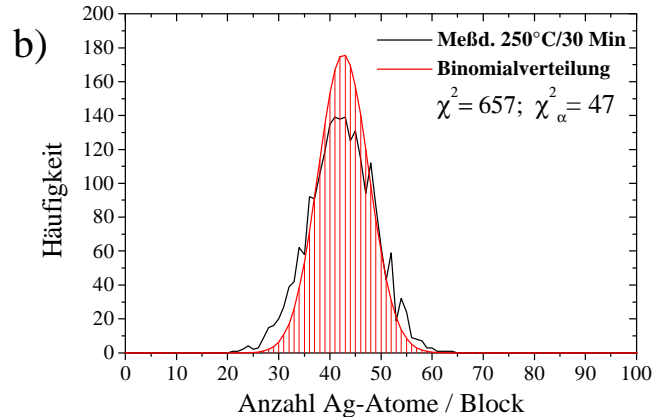

Abbildung 4-12: a) $\chi^{2}$-Test am Volumen der bei $200^{\circ} \mathrm{C} / 30$ Min getemperten Probe. b) $\chi^{2}-$ Test am Volumen der bei $250^{\circ} \mathrm{C} / 30$ Min getemperten Probe.

In einem Ausschnitt aus dem gesamten Meßvolumen sind deutlich runde Teilchen mit einem Durchmesser von 1 bis $2 \mathrm{~nm}$ zu erkennnen, deren $\mathrm{Ag}-$ Gehalt sich auf 60 at.- $\%$ beläuft. Der zugehörige $\chi^{2}-$ Test beweist ein stark inhomogenes Verhalten der Probe. Der gemessene $\chi^{2}-$ Wert, $\chi^{2}=657$, ist signifikant größer als der kritische Vergleichswert $\chi_{\alpha}{ }^{2}=47$. Interessanterweise steigt der mittlere Ag-Gehalt der Zwischenphase mit den Auslagerungsstufen stetig bis auf 42,3 at.- $\%$ Ag der jetzigen Probe $^{5}$. Es scheint so, daß die erwartete Bildung der $\gamma$-Gleichgewichtsphase durch langsames Einstellen der Gleichgewichtskonzentration von 60 at.-\% Ag über den Weg der Entmischung aus der zuvor gebildeten intermediären Phase verläuft.

$300^{\circ} \mathrm{C}$ für $30 \mathrm{~min}$. Dieses Bild wird durch die Analyse einer bei $300^{\circ} \mathrm{C}$ ausgelagerten Probe bestätigt. Es findet sich neben der restlichen Al-Schicht zum stöchiometrischen Ausgleich nur noch die Gleichgewichtsphase $\gamma$. Der $\chi^{2}-$ Test durch das Analysevolumen zeigt die Homogenität der gebildeten $\gamma$-Phase. Bei einer mittleren Konzentration von 58,2 at.- $\%$ Ag liegt der gemessene $\chi^{2}$-Wert von $\chi^{2}=21$ deutlich unter dem kritischen Vergleichswert $\chi_{\alpha}{ }^{2}=28$. Weitere Messungen bei gleichen Temperbedingungen zeigen ebenfalls die Bildung der $\gamma$-Phase bei ähnlichen Konzentrationen bis zu 64 at.-\% Ag. Schaut man sich die Rekonstruktion der erstgenannten Messung an, so existieren anscheinend Bereiche, in denen die Alund $\mathrm{Ag}$-Atome lagenweise angeordnet erscheinen (markiert in Abbildung 4-13 b). Das stöchiometrische Verhältnis der $\gamma$-Phase $\mathrm{Ag}_{2} \mathrm{Al}$ würde einer etwaigen Ordnung nicht widersprechen. Zur Prüfung der Hypothese, ob diese Bereiche eine Lagenordnung besitzen, müßte sich der Nachweis mit einem Konzentrationsprofil senkrecht zu den Netzebenen erbringen lassen. Der in Abbildung 4-13 e) wiedergegebene Konzentrationsverlauf wurde mit einem kleinen Analysezylinder

\footnotetext{
${ }^{5}$ Dies steht nicht im Widerspruch zu gleichen Ausgangsdicken der Al- und Ag-Lage. Entsprechend der eingestellten mittleren Konzentration besteht der Endzustand aus $\mathrm{Ag}_{2} \mathrm{Al}$ und restlicher unverbrauchter Al-Schicht an der Oberfläche.
} 
gezielt in dem markierten, vermutlich geordneten Bereich erstellt. Interessanterweise verläuft der $\mathrm{Ag}-$ Gehalt wie es für eine Lagenordnung zu erwarten war. Das Profil pendelt zwischen reiner Ag- und reiner Al-Lage von 100 auf 0 at.-\%. Diese Struktur läßt sich aber nur in Teilbereichen der Probe erkennen, während die anderen vermutlich so stark verkippt sind, daß die Fernordnung nicht mehr auffällt. In keiner anderen Probe, die ebenfalls diese Gleichgewichtsphase gebildet hat, wurde jedoch eine ähnliche Ordnungseinstellung festgestellt.

a)

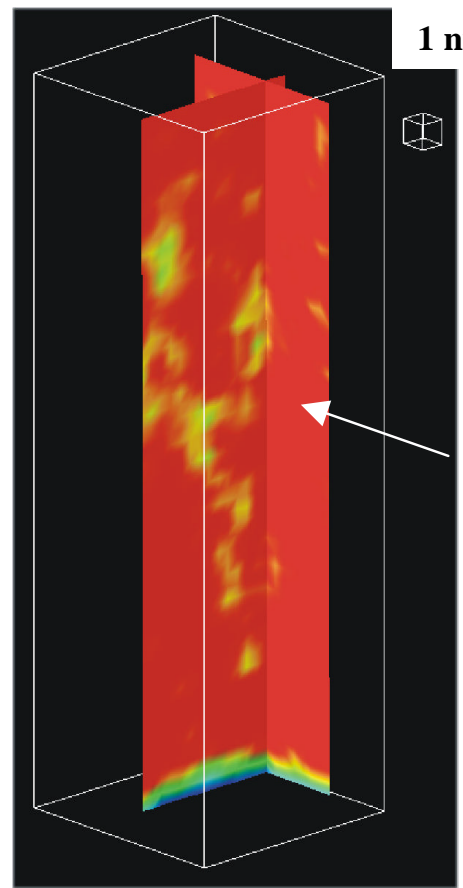

b)

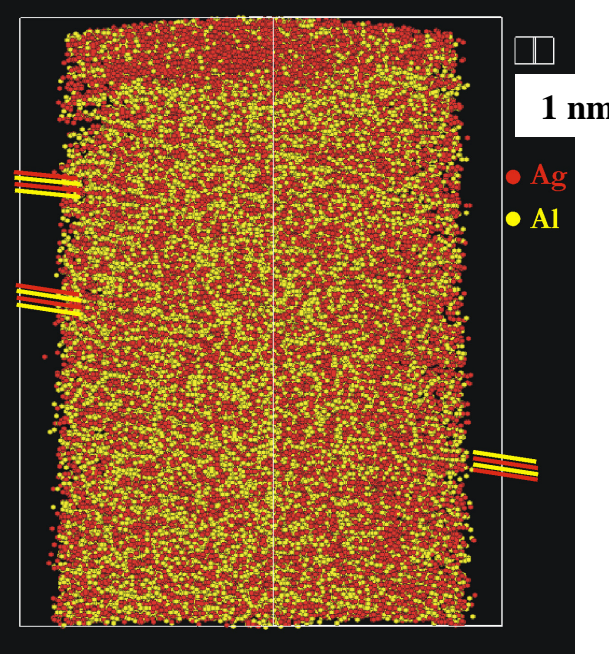

c)

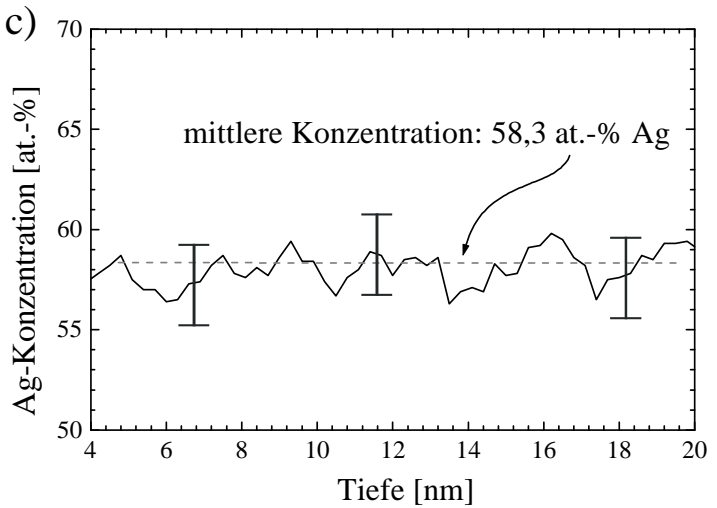

d)
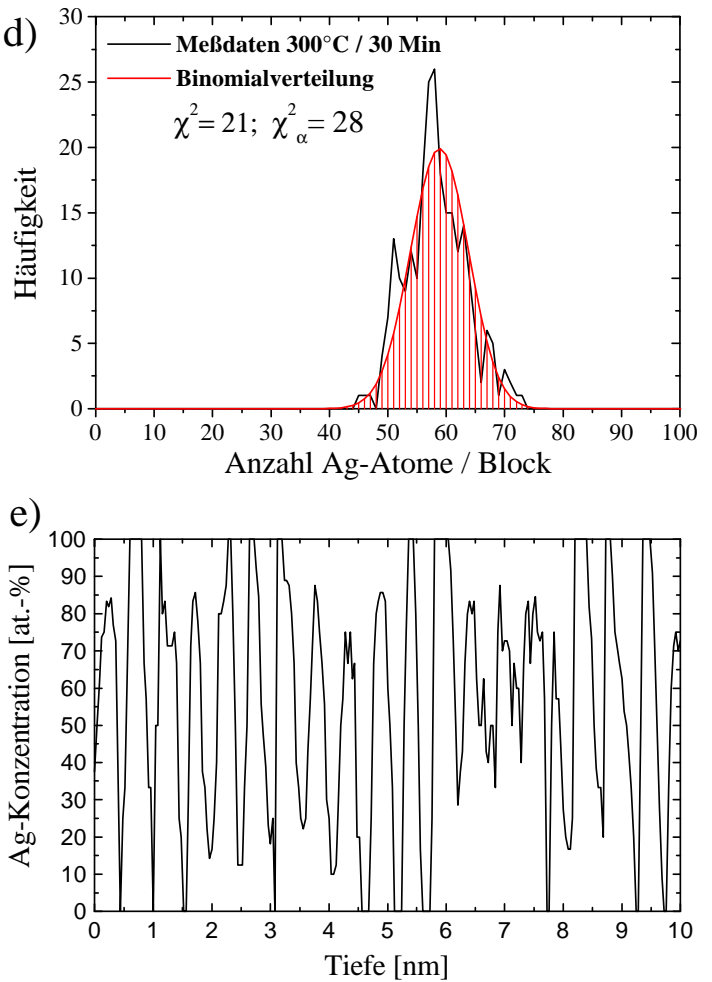

Abbildung 4-13: a) Tomographischer Schnitt durch das Volumen der bei $3_{00}^{\circ} \mathrm{C}$ wärmebehandelten Al/Ag-Probe. b) 3D-Rekonstruktion mit eingezeichneten Ordnungsstrukturen. c) Konzentrationsprofil durch das gesamte Meßvolumen. d) $\chi^{2}$-Test am Volumen der bei $300^{\circ} \mathrm{C} / 30$ Min getemperten Probe. e) Konzentrationsprofil über eingeschränkten Bereich der lagenweise geordneten Struktur. 
Die Messung der geordneten Phase ist dennoch durchaus als signifikantes Meßergebnis anzusehen, da es nicht durch einen Artefakt der Meßmethode erklärt werden kann. Die Bildung geordneter Bereiche wird sicherlich beeinflußt durch ganz spezielle Bedingungen beim Keimbildungs- und Wachstumsprozeß, die hier aber nicht näher erörtert werden sollen.

Was bei allen Ergebnissen der Analysen mit der Tomographische Atomsonde auffällt, ist der anscheinend einseitige Reaktionsverlauf. Die Messungen zeigen deutlich die starke Korngrenzendiffusion innerhalb der Ag-Schicht und die heterogene Keimbildung bevorzugt in den Tripelstellen. Innerhalb der Al-Schicht wurden dagegen weder Korngrenzen noch $\mathrm{Ag}$-benetzte Korngrenzen detektiert. Die Annahme liegt nahe, daß dies im Widerspruch zu den in Abschnitt 4.2 genannten Ergebnissen der Transmissionselektronenmikroskopie steht. Dort wurde eine nicht unbedeutende Eindiffusion von Ag in die Korngrenzen der Al-Schicht mit Hilfe der Z-Kontrast-Methode nachgewiesen. Man darf aber nicht aus den Augen verlieren, daß der Bereich des im TAP analysierten Probenvolumens mit etwa $10 \cdot 10 \cdot 100 \mathrm{~nm}^{3}$ Kantenlänge sehr viel kleiner ist, als die laterale Ausdehnung der im TEM untersuchten Proben. So ist schon der mittlere Abstand der in Abbildung 4-3 dargestellten Al-Korngrenzen erheblich größer als der vom TAP-Detektor abgedeckte Bereich. Deshalb ist auch die Wahrscheinlichkeit sehr gering eine Korngrenze im Meßvolumen $\mathrm{zu}$ finden. In unserem Fall zeigten schon die TEM-Aufnahmen der beschichteten Spitze eine Mindestkorngröße am Apex der Spitze für Al von $15 \mathrm{~nm}$, wohingegen sowohl die TEM Aufnahmen der planaren Schichten als auch die TAP-Ergebnisse für Ag eine ungefähre Korngröße zwischen 5 und $10 \mathrm{~nm}$ nachgewiesen haben. Gerade bei der Präparation der Schichten für die TAP-Analyse ist aufgrund des Präparationsverfahrens die Bildung einer Korngrenze auf dem Apex der Spitze ziemlich unwahrscheinlich. Daher konnte im Analysevolumen im Falle der abschließenden Al-Schicht keine Korngrenze detektiert werden.

Um diesen Fall aber zu erzwingen, wurde eine $6 \mathrm{~nm}$ dünne Al-Lage zwischen zwei Ag-Schichten der üblichen Dicke deponiert. Die Al-Kornstruktur muß aufgrund der geringen Schichtdicke ohne Zweifel feiner sein als in den vorher diskutierten Fällen.

$\mathrm{Ag} / \mathrm{Al}(6 \mathrm{~nm}) / \mathrm{Ag}-150^{\circ} \mathrm{C}$ für $\mathbf{3 0}$ Minuten. Eine spezielle Schwierigkeit in der Messung solcher Schichtfolgen liegt in der unterschiedlichen Feldverdampfungsfeldstärke der beteiligten Materialien. Wie in Tabelle 2 aufgelistet, liegt der Wert für Al unter dem von Ag. Für die Stabilität der Schichten während der Messung ist es jedoch grundsätzlich empfehlenswert, die Analyse von Materialien niedriger Feldverdampfungsfeldstärke zu solchen hoher Feldverdampfungsfeldstärke verlaufen $\mathrm{zu}$ lassen, wie es bisher in der Abfolge $\mathrm{Al} \rightarrow \mathrm{Ag} \rightarrow \mathrm{W}$ der Fall war. Deshalb war die Zahl erfolgreicher Messungen der physikalisch sehr reizvollen aber meßtechnisch eher schwierigen Schichtfolge sehr gering. In der Regel erfolgt ein kompletter Abriß des verbliebenen Schichtpaketes beim Übergang von der ersten Ag-Lage in die Al-Schicht. Dennoch sind zwei Messungen erfolgreich durch den gesamten Trilayer 
bis in das Substrat verlaufen. Abbildung 4-14 a) zeigt einen tomographischen Schnitt durch das bei $150^{\circ} \mathrm{C}$ für 30 Minuten ausgelagerte $\mathrm{Ag} / \mathrm{Al} / \mathrm{Ag}-\mathrm{W}$ Schichtpaket. Zusätzlich sind noch die Al-Atome des Meßvolumens mit eingezeichnet. In diesem Fall geht die farbliche Markierung von rot: 100 at.- $\%$ Ag zu blau: 100 at.- $\%$ Al. Al-Eindiffusion in die $\mathrm{Ag}$-Korngrenzen und Bildung der vorher diskutierten intermediären Phase $\mathrm{Al}_{2} \mathrm{Ag}$ ist in diesem Probenbereich nicht $\mathrm{zu}$ beobachten. Wie erwartet ist die Kornstruktur der dünnen Al-Lage deutlich feiner als in der vorherigen Bilayer-Stapelfolge. Innerhalb der Al-Schicht läßt sich eine Korngrenze erkennen, die durch starke Ag-Anreicherung deutlich hervortritt. Ein Konzentrationsprofil senkrecht zu dieser Segregationszone mit einem Radius des Analysezylinders von $1 \mathrm{~nm}$ ermöglicht die genaue Bestimmung des Ag-Gehaltes. Überraschenderweise stellt sich bereits eine Konzentration von 60 at.-\% Ag innerhalb der Al-Korngrenze ein, welche genau der Gleichgewichtsphase $\gamma-\mathrm{Ag}_{2} \mathrm{Al}$ entspricht. Anscheinend erfolgt beim Reaktionsverlauf innerhalb der Al-Korngrenze die Bildung der Gleichgewichtsphase $\gamma$ direkt, ohne den Zwischenschritt der Bildung der intermediären Phase $\mathrm{Al}_{2} \mathrm{Ag}$, wie innerhalb der $\mathrm{Ag}-$ Korngrenzen. Die Breite des Konzentrationsprofils liegt bei $2 \mathrm{~nm}$, so daß hier schon von einer gebildeten Phase gesprochen werden kann, die sich in Richtung der Al-Körner weiter entwickelt.
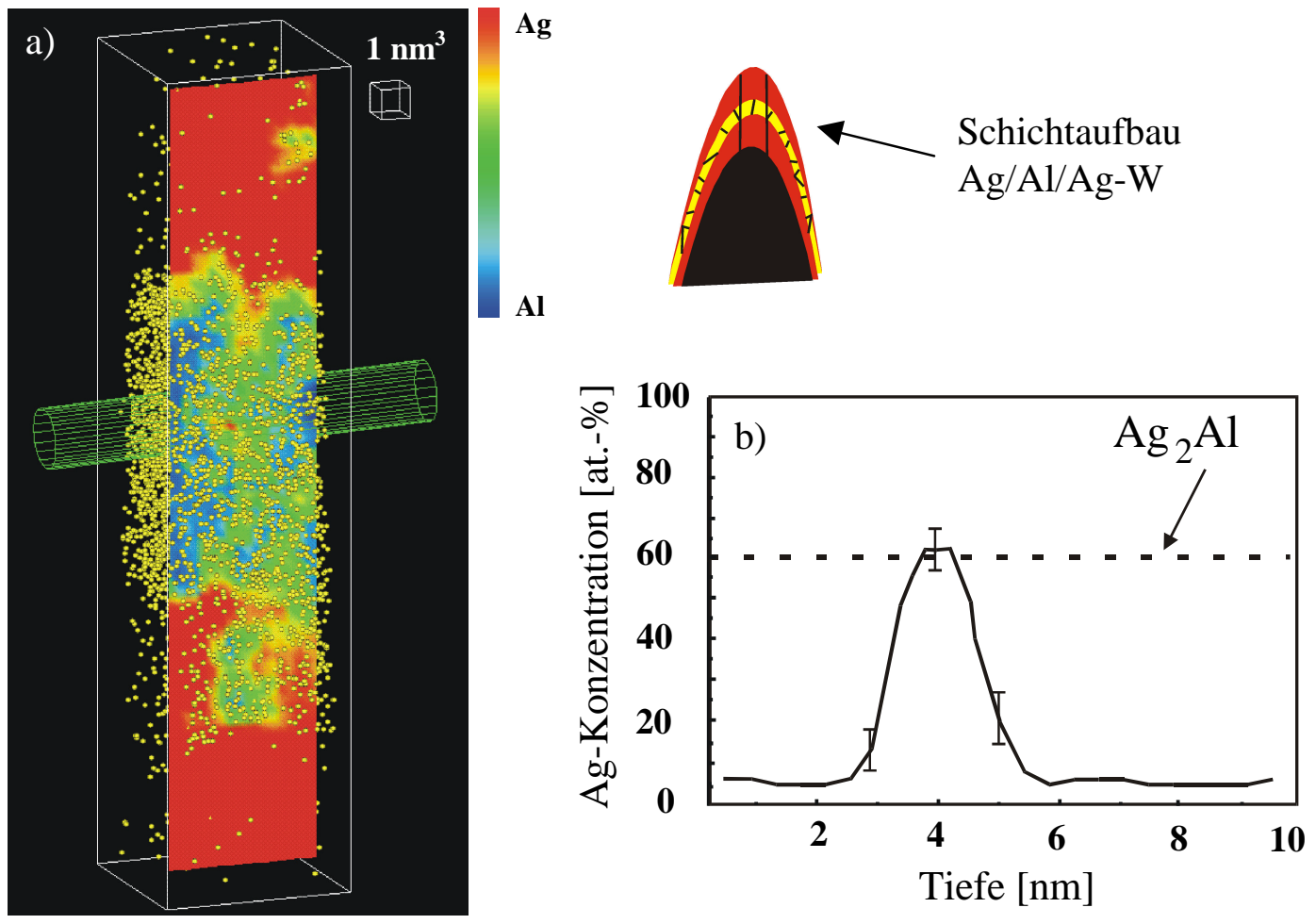

Abbildung 4-14: a) Tomographischer Schnitt durch die Mitte des Volumens einer Ag/Al/Ag-Probe die bei ${ }^{150} \mathrm{C}$ für 30 Minuten wärmebehandelt wurde. Die Fehlfarbenmarkierung läuft von rot: 100 at.-\% Ag zu blau: 100 at.-\% Al. Zusätzlich sind die Al-Atome mit eingezeichnet. b) Konzentrationsanalyse entlang des in Teilbild a) eingezeichneten Zylinders, senkrecht zur Korngrenze orientiert. 
Bemerkenswert ist in diesem Experiment die Reaktionsgeschwindigkeit. Vergleicht man die Segregationszone von $2 \mathrm{~nm}$ mit der Breite der gebildeten $\mathrm{Al}_{2} \mathrm{Ag}-\mathrm{Phase}$ der bei $140^{\circ} \mathrm{C}$ für 30 Minuten ausgelagerten $\mathrm{Al} / \mathrm{Ag}-P r o b e$, die $40 \mathrm{~nm}$ beträgt, so fällt ein deutlicher Unterschied in der Reaktionskinetik auf. Die direkte Bildung der Gleichgewichtsphase innerhalb der Al-Korngrenzen verläuft wesentlich langsamer als der Prozeß der Al-Eindiffusion in die $\mathrm{Ag}-$ Korngrenzen und Bildung der $\mathrm{Al}_{2} \mathrm{Ag}-$ Phase unter Aufzehrung der restlichen Ag-Körner.

\subsection{Diskussion der Ergebnisse}

Die vorgestellten Ergebnisse zeigen eindrucksvoll die Möglichkeiten der angewandten Methode. Dank der chemischen Information auf kleinster Skala konnte ein vom planaren Reaktionsverhalten abweichender Prozeß aufgezeigt werden. Der Ablauf der Reaktion ist dabei eindeutig gesteuert durch die vorliegende Mikrostruktur der Probe. Es entwickelt sich eine dreidimensionale Prozeßmorphologie, die mit anderen Methoden nicht in dieser Genauigkeit charakterisiert werden konnte. Nach dem Stand der Literatur wurde die Bildung einer intermediären Phase als Vorstadium zur Bildung der $\gamma$-Gleichgewichtsphase bisher noch nicht nachgewiesen, da die zuvor benutzten chemischen Analysemethoden zu grob waren. Bereits ab einer Wärmebehandlung von $90^{\circ} \mathrm{C}$ zeigt sich eine erstaunliche Asymmetrie im Reaktionsverlauf der Ag- und Al-Schichten. Auf der Al-Seite hat sich eine $2 \mathrm{~nm}$ ausgedehnte Interdiffusionszone gebildet, während auf der Ag-Seite nur auf einer Länge von $1 \mathrm{~nm}$ Durchmischung stattfand.

\subsubsection{Diffusionsverhalten des planaren Reaktionsstadiums}

Die Interdiffusionszone ist mit $2 \mathrm{~nm}$ Breite innerhalb der Al-Schicht doppelt so weit ausgedehnt wie die $1 \mathrm{~nm}$ Zone in der Ag-Lage. Wie im experimentellen Abschnitt erwähnt, sind die aufgrund reiner Volumendiffusion berechneten erreichbaren Längen insbesondere auf der $\mathrm{Ag}-$ Seite sehr viel geringer. Eine Möglichkeit der quantitativen Kontrolle ist die Ermittlung der beobachteten Diffusionskoeffizienten. Dies geschieht durch Anpassen der Dickschichtlösung mit einem konzentrationsunabhängigen Diffusionskoeffizienten D an die Konzentrationsprofile:

$$
c(x, t)=\frac{c_{0}}{2} \cdot \operatorname{erf}\left(\frac{x}{2 \sqrt{D t}}\right)
$$

Um das Konzentrationsprofil des Ausgangszustandes zu berïcksichtigen, wurde ein Ansatz nach HALL benutzt [HALL]. Dort wird ein um die Anfangsbedingung modifizierter Diffusionskoeffizient D angesetzt:

$$
4 D t=4 D_{\exp } t-a^{2}
$$


$D_{\text {exp }}$ ist der experimentell ermittelte Diffusionskoeffizient und der Parameter a ergibt sich aus der Funktion erf(x/a), die den unreagierten Ausgangszustand beschreibt. Die so berechneten Konzentrationsverläufe sind zusätzlich $\mathrm{zu}$ den Meßdaten in Abbildung 4-15 dargestellt.

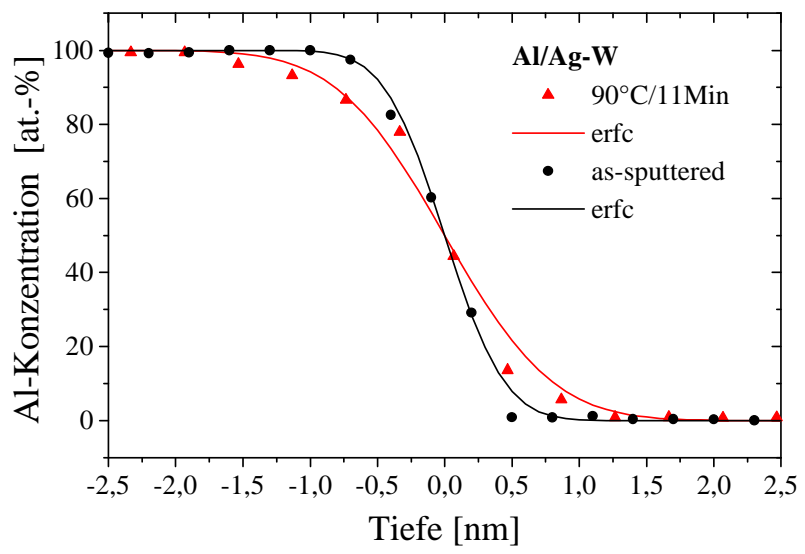

\begin{abstract}
Abbildung 4-15: Konzentrationsprofile an der Al/Ag-Grenzfläche im Ausgangszustand und nach einer Auslagerung bei $90^{\circ} \mathrm{C}$ für 11 Minuten. Die durchgezogenen Linien ergeben sich aus einer Anpassung der Dickschichtlösung nach Gl.(4.1).
\end{abstract}

Die Kurve des unreagierten Zustandes zur Ermittlung von $D_{\text {exp }}$ läßt sich mit geringem Fehler anpassen. Bei der getemperten Probe macht sich die Asymmetrie allerdings stark bemerkbar. Beschränkt man sich bei der Anpassung auf den Konzentrationsverlauf innerhalb der Al-Schicht, so erhält man einen Diffusionskoeffizienten von $\mathrm{D}=2,410^{-18} \mathrm{~cm}^{2} \mathrm{~s}^{-1}$. Vergleicht man diesen mit dem auf die entsprechende Temperatur extrapolierten Literatur-DK, mit den Parametern aus Tabelle 3, so zeigt sich eine Abweichung von nicht mal $35 \%$. Bedenkt man die Ungenauigkeiten bei der Anpassung, so ist dieser Wert schon ein deutliches Indiz für eine volumendiffusions-dominierende Reaktion auf der Al-Seite.

Im Vergleich dazu, läßt sich der Konzentrationsverlauf innerhalb der Ag-Schicht nicht sinnvoll anpassen. Zwischen experimentell ermittelten DK und Literaturwert liegen mehrere Größenordnungen, so daß hier andere reaktionsbestimmende Faktoren zu vermuten sind. Zum einen kann die bei späteren Auslagerungszuständen deutlich hervortretende Al-Eindiffusion in die Korngrenzen der Ag-Schicht schon im Anfangsstadium vorhanden sein - zum anderen besteht die Möglichkeit, daß durch das Depositionsverfahren speziell in der Grenzfläche zur Ag-Lage eine entsprechend erhöhte Anzahl an Defekten eingebaut wurde. Dies würde bei Wärmebehandlungen den beobachteten katalytischen Effekt verursachen, wie er auch schon von WEAVER angedeutet wurde [WEAVER].

Als sicher anzusehen, ist das Verständnis des Reaktionsverlaufes auf der Al-Seite. Dieser ist, bedingt durch die größeren Al-Körner, hauptsächlich durch Volumendiffusion bestimmt. 


\begin{tabular}{|l|c|c|c|c|}
\hline & $\mathbf{A g}$ in $\mathbf{A g}^{\mathbf{6}}$ & $\mathbf{A g}$ in $\mathbf{A l}^{\mathbf{7}}$ & $\mathbf{A l}$ in $\mathbf{A g}^{\mathbf{8}}$ & $\mathbf{A l}$ in $\mathbf{A l}$ \\
\hline $\mathbf{D}_{\mathbf{0}}\left[\mathbf{1 0}^{-4} \mathbf{~ m}^{\mathbf{2}} \mathbf{s}^{-1}\right]$ & 0,041 & 0,13 & 0,13 & 0,176 \\
\hline $\mathbf{Q}\left[\mathbf{k J ~ m o l}^{-\mathbf{1}}\right]$ & 169,8 & 117,2 & 159,5 & 126,4 \\
\hline
\end{tabular}

Tabelle 3: Diffusionsparameter der Volumendiffusion

Der weitere Reaktionsverlauf bei höheren Temperaturen ist bestimmt durch $\mathrm{Al}$-Korngrenzendiffusion und Bildung einer intermediären Phase $\mathrm{Al}_{2} \mathrm{Ag}$. Die eigentlich erwartete Gleichgewichtsphase $\gamma$ mit einer Stöchiometrie von $\mathrm{Ag}_{2} \mathrm{Al}$ bildet sich durch Ausscheidung aus der Zwischenphase. Um diesen Bildungsweg zu verstehen, ist eine genaue Kenntnis der Struktur der intermediären Phase notwendig.

\subsubsection{Struktur der intermediären Phase $\mathrm{Al}_{2} \mathrm{Ag}$}

Die Frage nach der Struktur der gemessenen Zwischenphase offenbart die Grenzen der Tomographischen Atomsonde. Zwar sind chemische Analysen auf atomarer Genauigkeit möglich, jedoch läßt sich aus den Rekonstruktionen der Meßdaten auch durch computerunterstützte Auswertung aufgrund der Auflösungsgrenze von etwa $2 \AA$ keine Aussage über die vorliegende Struktur machen. Strukturelle Information könnte aber die feldionenmikroskopische Abbildung der Phase liefern. Wie allerdings bereits in Abschnitt 4.3.1 erläutert, ist die Abbildung der reinen Al-Schicht nahezu unmöglich. In der Regel gelingt deren Projektion, wenn geringe Anteile anderer Komponenten enthalten sind. Daher war zu vermuten, daß die Charakterisierung der $\mathrm{Al}_{2} \mathrm{Ag}$-Phase Erfolg haben könnte. Aber dennoch zeigte sich an einer bei $140^{\circ} \mathrm{C}$ für 30 Minuten ausgelagerten Probe, daß weder die strukturell reine $\mathrm{Al}-$ noch die $\mathrm{Al}_{2} \mathrm{Ag}-\mathrm{Schicht}$, die etwa eine Dicke von $40 \mathrm{~nm}$ haben sollte, abzubilden war. In Abbildung 4-16 ist die Kornstruktur der Ag-Lage dargestellt. Am Apex der Spitze befindet sich ein dreieckiges Korn mit durch den (111)-Pol laufender Schraubenversetzung. In den andern Bildbereichen sind Körner mit einer Größe zwischen 5 und $15 \mathrm{~nm}$ sichtbar. Dazwischen zeigen sich deutlich dunkle Bereiche, die auch bei erhöhter Grundspannung nicht abgebildet werden konnten. Aufgrund ihrer Breite von über $2 \mathrm{~nm}$ handelt sich hierbei um die Segregationszonen, in denen sich die $\mathrm{Al}_{2} \mathrm{Ag}-\mathrm{Phase}$ gebildet hat.

Da sich auch auf diesem Wege keine Erkenntnis über die Struktur der intermediären Phase erschlossen hat, ist das Heranziehen und Interpretieren von Meßergebnissen anderer strukturauflösender Methoden notwendig.

\footnotetext{
${ }^{6}$ vgl. [LAM]

${ }^{7} \mathrm{vgl}$. [ALEXANDER]

${ }^{8} \mathrm{vgl}$. [FOGELSON]

${ }^{9}$ vgl. [VOLIN]
} 


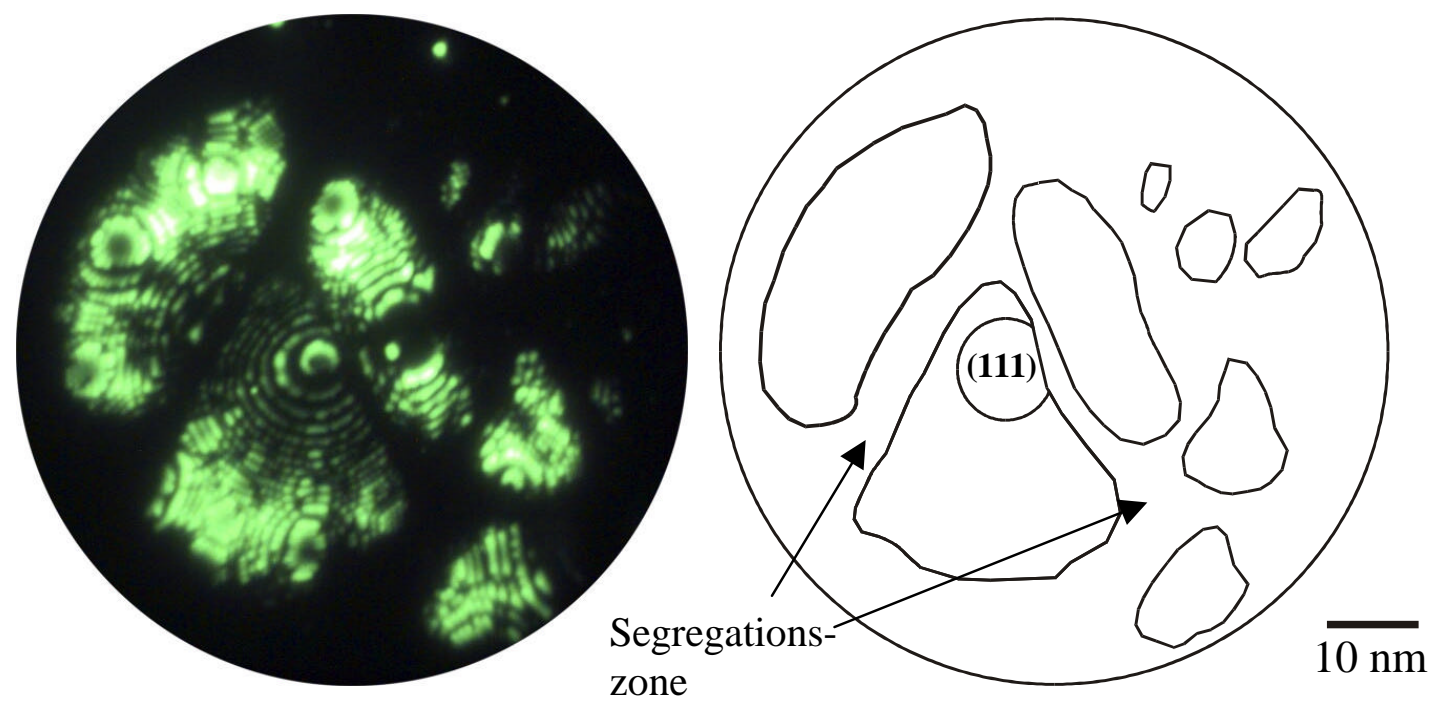

Abbildung 4-16: Feldionenmikroskopische Abbildung der gesputterten Ag-Schicht nach einer Wärmebehandlung bei $140^{\circ} \mathrm{C}$ für 30 Minuten.

In der Literatur existieren bereits einige XRD-Untersuchungen zu Al/Ag-Schichtsystemen [BAGLIN, Roy, Schmitz1]. Die Kernaussage dieser Untersuchungen ist, daß neben den fcc-Reflexen bei einsetzender Schichtreaktion nur die hcp-Reflexe der neu gebildeten Gleichgewichtsphase $\mathrm{Ag}_{2} \mathrm{Al}$ auftreten. Dies wird durch kürzlich erzielte elektronenmikroskopische Beugungsaufnahmen bestätigt [Svenson]. Da diese Daten für die Interpretation der TAP-Ergebnisse eine hohe Relevanz haben, wurden erneut Heizexperimente in einer dafür ausgelegten XRD-Anlage durchgeführt. Um einen direkten Bezug zu den im TAP untersuchten Schichten $\mathrm{zu}$ haben, wurden Multilayer mit gleichen Schichtdicken und Depositionsraten auf planare $\mathrm{Si}$-Substrate deponiert. Anschließend erfolgte die isotherme Auslagerung unter ständiger Aufnahme von Diffraktogrammen. In Abbildung 4-17 a) ist die zeitliche Entwicklung eines solchen $\mathrm{Al} / \mathrm{Ag}$-Schichtpaketes bei einer Temperatur von $115^{\circ} \mathrm{C}$ gezeigt. Bereits nach 30 Minuten zeigen sich neben dem fcc (111)-Reflex erste Peaks der hexagonalen Phase. Außer diesen fcc- und hcp-zugehörigen Reflexen treten keine anderen Strukturreflexe auf, so daß im Umkehrschluß die in den TAP-Untersuchungen gefundene Phase $\mathrm{Al}_{2} \mathrm{Ag}$ entweder fcc- oder hcp-Struktur haben muß. Auch für Ordnungstendenzen gibt es keine Hinweise, da keine Überstrukturpeaks auftreten. Eine abweichende Konzentration der intermediären Phase zur Gleichgewichtsphase $\gamma$ ist in Bezug auf die Diffraktogramme kein Widerspruch. Aufgrund gleicher Gitterkonstanten von Ag und Al verursachen Konzentrationsänderungen innerhalb der gebildeten Phase keine Verschiebung der Röntgenreflexe. 

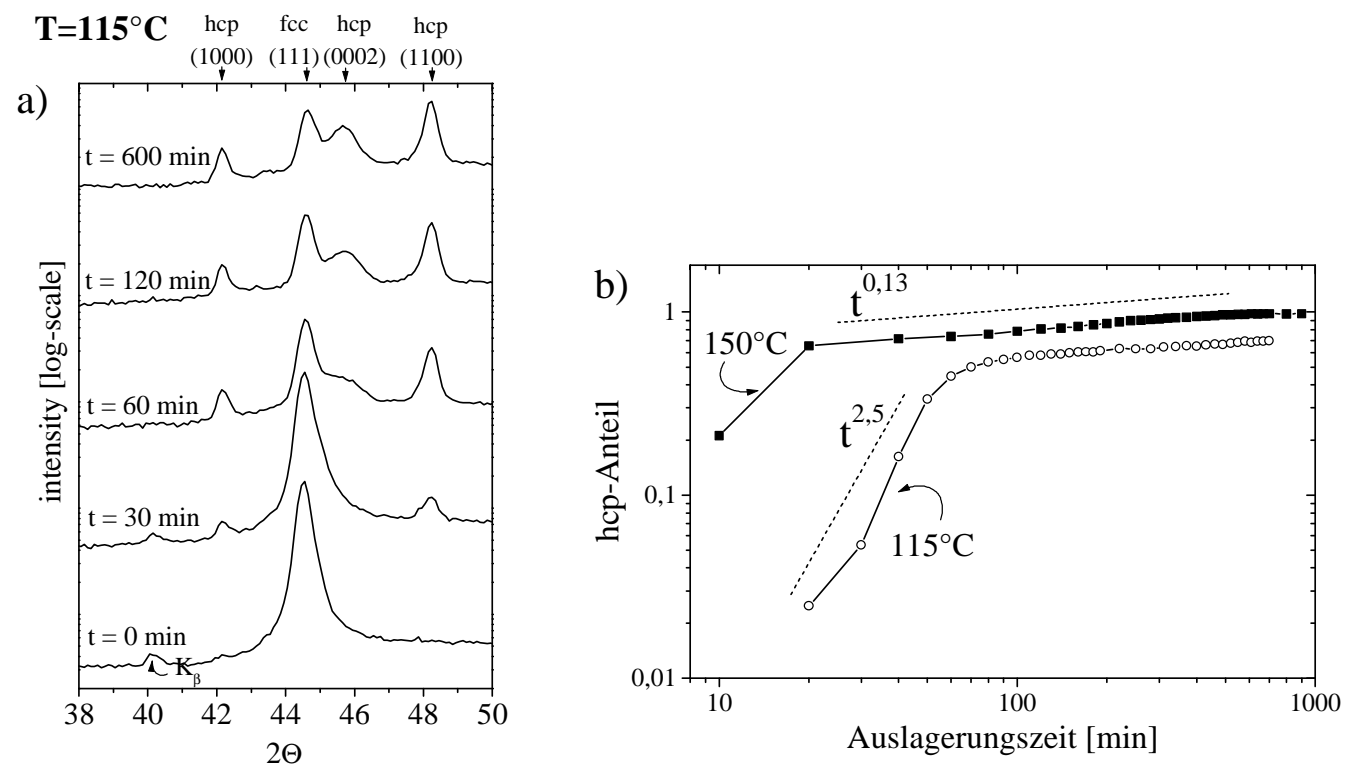

Abbildung 4-17: Ergebnisse der XRD-Heizexperimente an $\mathrm{Al}_{30 \mathrm{~nm}} / \mathrm{Ag}_{30 \mathrm{~nm}}$ Schichtsystemen; a) XRD-Spektren bei isothermer Auslagerung von $115^{\circ} \mathrm{C}$ für verschiedene Zeiten; b) Entwicklung des gebildeten hcp-Phasenanteils im Laufe der Zeit für $\mathrm{T}=115^{\circ} \mathrm{C}$ und $\mathrm{T}=150^{\circ} \mathrm{C}$

Über die auftretenden Intensitäten der Röntgenreflexe läßt sich der Anteil der gebildeten hcp-Phase berechnen. Die zeitliche Entwicklung des Phasenanteils ist in Abbildung 4-17 b) für zwei verschiedene Auslagerungstemperaturen dargestellt. Auffällig bei beiden ist die Trennung des Reaktionsablaufes in zwei Geschwindigkeitsregime. Die Reaktion startet sehr schnell, mit einem Zeitexponenten von etwa 2,5 und geht dann in einen langsam auslaufenden Modus über. Die Reaktionkinetik scheint bei niedrigen Temperaturen derart gehemmt zu sein, daß die Interreaktion nur durch Erhöhung der Temperatur auf $150^{\circ} \mathrm{C}$ in endlicher Zeit zum Abschluß kommt.

Wie bereits erwähnt wurde, ist aus der Literatur eine metastabile Mischungslücke in übersättigten $\mathrm{Al}-\mathrm{Ag}-$ Legierungen bekannt. Je nach Entmischungstemperatur wird die Bildung von zwei unterschiedlichen metastabilen Guinier-Preston-Zonen mit fcc-Sruktur [BAUR2] beobachtet. Die silberreiche Zone hat einen Ag-Gehalt von 60 at.- $\%$ und wird in der Literatur als $\eta$-Ausscheidung bezeichnet. Die andere mit $\varepsilon$ gekennzeichnete GP-Zone liegt mit ihrer Konzentration bei 30 at.-\% Ag. Beide Zonen bilden vollkommen kohärente Grenzflächen mit dem $\alpha$-Mischkristall, mit einer Fehlpassung von weniger als 0,3\% [UnGÁR]. Aufgrund von thermodynamischen Überlegungen wird für die $\eta$-Phase mit 60 at.-\% Ag eine geordnete Struktur vorgeschlagen [HILLERT].

Es erscheint also sinnvoll für die gefundene intermediäre Phase eine fcc-Struktur zu postulieren, wie es in [SCHLEIWIES2] bereits vorgeschlagen wurde. Allerdings sprechen zwei gewichtige Gründe gegen diese Interpretation:

a) Bei einer zwangsläufigen Umwandlung von fcc- zu hcp-Struktur wäre ein kontinuierlicher Übergang der gebildeten intermediären Phase hin zur Gleichgewichtsphase $\gamma$, wie er beobachtet wurde, weitgehend unwahrscheinlich. 
b) Der direkte Vergleich des umgewandelten hop-Phasenanteils der Röntgenexperimente mit den TAP-Messungen spricht eindeutig für eine hcp-Struktur. Nach einer 30 minütigen Auslagerung zeigt das XRD-Experiment einen hcp-Phasenanteil von $70 \%$. Vergleicht man dies mit der TAP-Analyse der bei $140^{\circ} \mathrm{C}$ ausgelagerten Probe, so zeigt sich dort nur die über $40 \mathrm{~nm}$ ausgedehnte intermediäre Phase mit 33 at.-\% Ag. Für die gleichzeitige Existenz der erwarteten Gleichgewichtsphase $\gamma$ gibt es keinerlei experimentellen Nachweis. Zusätzlich läßt sich noch die TAP-Messung der bei $200^{\circ} \mathrm{C}$ getemperten Spitze zum Vergleich hinzuziehen. Die Auslagerungstemperatur liegt höher als bei der XRD-Messung und müßte demnach deutlich die hcp $\gamma$-Phase zeigen. Man findet aber nur eine vollständig durchreagierte Probe mit einer mittleren Konzentration bei 30 at.-\% Ag. Auch hier ist kein Bereich mit einer der $\gamma$-Phase zugehörigen Konzentration von $\mathrm{Ag}_{2} \mathrm{Al}$ nachzuweisen. Da die Röntgenmessungen ausschließlich die fcc-Reflexe der reinen Komponenten und die hcp-Peaks der Produktphase zeigen, bleibt nur der Schluß übrig, daß die gebildete intermediäre Phase $\mathrm{Al}_{2} \mathrm{Ag}$ hcp-Struktur haben muß. Dies hat natürlich für das Verständnis des Reaktionsablaufes weitreichende Konsequenzen. Demnach wird in der Tat die nach dem Gleichgewichtsphasendiagramm erwartete und nach der Literatur gefundene hexagonale Struktur während der Interreaktion gebildet. Deren Konzentration liegt mit einem $\mathrm{Al} \mathrm{zu} \mathrm{Ag}$ Verhältnis von 2:1 jedoch weit weg vom Gleichgewicht. Diese Konzentrationsdifferenz wird vermutlich, wie schon erwähnt, aufgrund kinetischer Aspekte stabilisiert. Erst bei höheren Temperaturen kann dann die Einstellung der Gleichgewichtskonzentration erfolgen.

Wirklich erstaunlich ist der Aspekt, daß die intermediäre Vorläuferphase die gleiche Struktur wie die Gleichgewichtsphase hat. Üblicherweise sind die metastabilen Zwischenstrukturen unterschiedlich, wie Arbeiten zur Festkörperamorphisierung am Beispiel von Fe/Zr [Aubertin, Krebs1, KREBS2] deutlich machen.

An dieser Stelle muß noch einmal betont werden, daß die Herleitung der Struktur auf einer reinen Korrelation der Meßergebnisse unterschiedlicher Methoden beruht. Ein direkter experimenteller Nachweis durch strukturauflösende Methoden wie Röntgendiffraktometrie an den spitzenförmigen Proben ist nicht möglich. Dennoch kann mit großer Sicherheit durch Vergleich der sehr genauen Ergebnisse auf eine hexagonale Struktur der gebildeten Produktphase geschlossen werden.

Im nächsten Schritt ist nun zu klären, wie es möglich ist, daß die hexagonale Phase weit weg von ihrer eigentlichen Gleichgewichtskonzentration auftritt. Den leichtesten Zugang zur Klärung der Frage bietet die Betrachtung der Thermodynamik des Systems anhand Freier Energie-Kurven.

\subsubsection{Thermodynamische Eigenschaften des Systems}

Bei einer angenommenen Stabilisierung der hcp-Phase bei $\mathrm{Al}_{2} \mathrm{Ag}$ müßte die Freie Energie im entsprechenden Konzentrationsbereich niedriger als die fcc-Phase sein. Wie bereits erwähnt, ist aus experimentellen Daten eine temperaturabhängige metastabile Mischungslücke in der fcc-Struktur in der Literatur bekannt. Abhängig von der Temperatur bilden sich GP-Zonen von 35 at.-\% Ag bis zu 55 at.-\% Ag mit 
fcc-Struktur. Die Freie Energie Kurve der fcc-Phase muß eine Entmischungstendenz im entsprechenden Temperaturbereich haben. Da fcc und hcp ähnlich in der Koordination ihrer Struktur sind, ist zu vermuten, daß der Verlauf der Freien Energien jenseits des hcp-Stabilitätsbereiches ähnlich sein sollte. Insbesondere kann so die Existenz einer Mischungslücke der hcp-Kurve neben der Entmischungstendenz der fcc-Phase postuliert werden.

Diese Arbeitshypothese findet sich allerdings nicht in den berechneten Freien Energien von LIM wieder [LIM]. In Abbildung 4-18 sind die berechneten Freien Energien der fcc- und hcp-Phase im thermodynamischen Gleichgewicht bei einer angenommenen Temperatur von $600 \mathrm{~K}$ dargestellt. Zur Kalkulation wurde Gl.(4.3) benutzt. Der letzte Term $\mathrm{G}^{\mathrm{E}}$ steht für einen Polynom Ansatz der Exzess-Energie nach RedLiCH-KisteR-Muggianu. Für $\mathrm{G}^{\mathrm{E}}=0$ beschreiben die übrigen Terme eine ideale Lösung [HAASEN].

$$
G^{\text {Phase }}(T, c)=c_{a} G_{a}^{\text {Phase }}(T)+c_{b} G_{b}^{\text {Phase }}(T)+R T\left[c_{a} \ln c_{a}+c_{b} \ln c_{b}\right]+G^{E}
$$

Verfolgt man den Verlauf der fcc-Kurve, so fällt auf der Al-reichen Seite eine schwache Tendenz zur Entmischung auf. Die gesuchte Ähnlichkeit der hcp-Kurve zum fcc-Verlauf ist jedoch nicht festzustellen. Sie zeigt im Gegenteil nur für den Bereich der Gleichgewichtskonzentration eine stabile Phase an.

Wie ist diese Diskrepanz zur aufgebauten Vorstellung zu erklären? Ein Blick auf die zur Berechnung benutzten experimentell gewonnen thermodynamischen Daten gibt Hinweise auf die möglichen Ursachen. Zur Beschreibung der reinen Ag- und Al-, sowie der hexagonalen $\gamma$-Phase wurden die von WITTIG durch Umlösungsexperimente bestimmten Bildungsenthalpien benutzt [WITTIG]. Am auffälligsten ist die Tatsache, daß innerhalb des interessanten Konzentrationsbereiches von 50 at.$\% \mathrm{Al}$ bis 80 at.-\% Al keine Meßwerte aufgenommen wurden! Die Autoren beschreiben durchgeführte Experimente in diesem Konzentrationsbereich, die durch stark negative Bildungswärmen und durch Nicht-Gleichgewichtseinstellung selbst nach 7 Tagen auf metastabile Zustände hindeuten und deshalb nicht tabelliert wurden. Es wird die Vermutung geäußert, daß aufgrund entgegengesetzten Steigungsverhaltens von Lösungs- und Bildungswärme die Bildung der Gleichgewichtsphase $\gamma$ jenseits des Stabilitätsbereiches möglich ist. Es muß auch erwähnt werden, daß diese Experimente bei $470^{\circ} \mathrm{C}$ durchgeführt wurden, was deutlich über dem Temperaturbereich der im TAP erstmals bei $115^{\circ} \mathrm{C}$ detektierten $\mathrm{Al}_{2} \mathrm{Ag}-\mathrm{Phase}$ liegt.

Neben den eben erwähnten Bildungsenthalpien wurden von LIM zusätzlich Aktivitätsdaten verwendet, die aus EMK-Messungen gewonnen wurden [HILLERT]. Auch an dieser Stelle muß festgestellt werden, daß in dem Al-reichen Konzentrationsbereich keine Meßwerte vorhanden sind. Ein Vergleich der EMK-Daten mit den Lösungsenthalpien von WITTIG zeigt zudem eine deutliche Abweichung voneinander, was als keine definierte Einstellung des Gleichgewichts bei den EMK-Messungen gedeutet wird [WITTIG]. 
a)

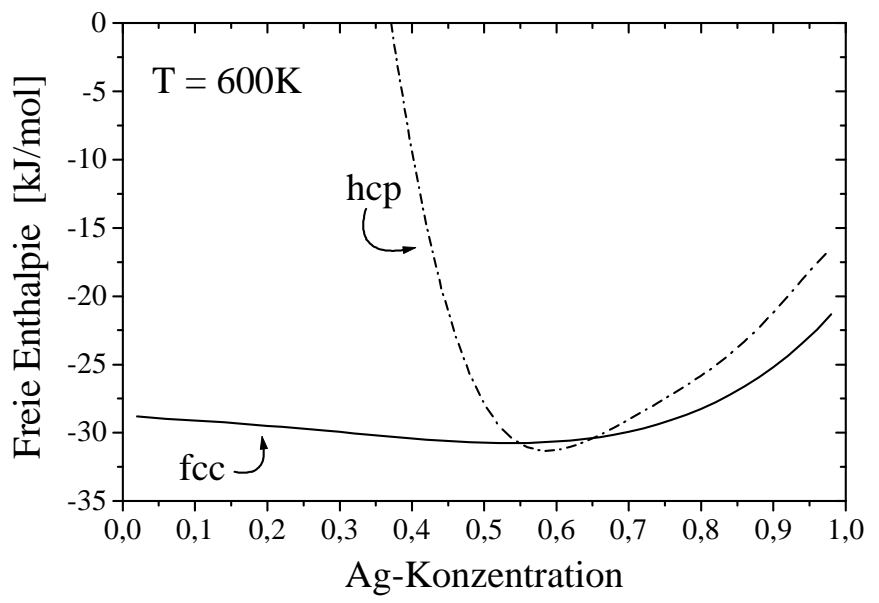

b)

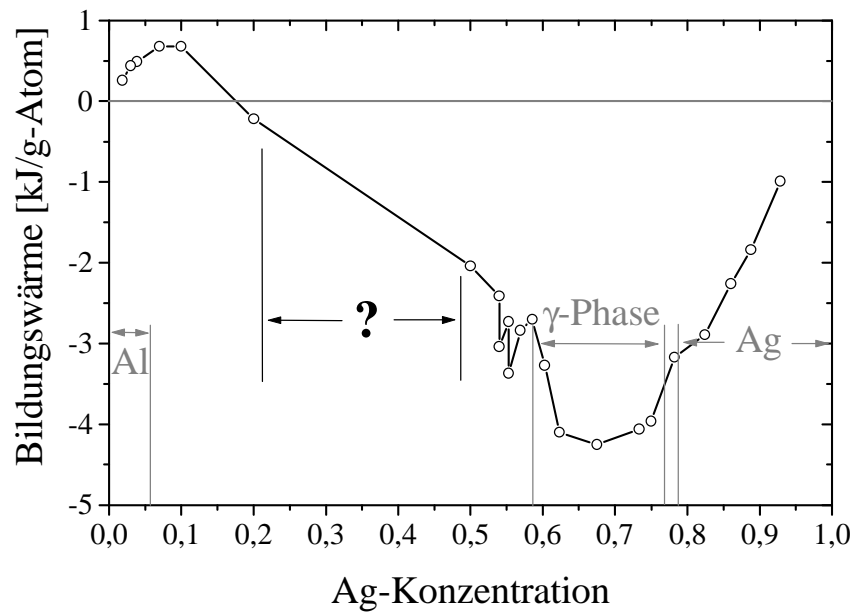

Abbildung 4-18: a) Freie Energie Kurven nach [LIM] und b) die zur Berechnung benutzten experimentell bestimmten Bildungswärmen [WITTIG].

Aufgrund von störenden Effekten, wie Konzentrationsänderungen an der Oberfläche der Probe oder Kriechströme sind die Meßwerte zudem mit einem großen Fehler behaftet. Die Temperaturen der EMK-Experimente liegen bei ca. $800 \mathrm{~K}$ und sind somit weit über den in dieser Arbeit benutzten Temperaturen.

In der Summe muß also festgestellt werden, daß die Freien Energie Kurven von LIM nicht zur Beschreibung der Phasenbildung in unserem Temperatur- und Konzentrationsbereich geeignet sind, da die verwendeten thermodynamischen Daten in diesem Bereich mangelhaft sind, bzw. nicht vorliegen. Auf eine saubere Beschreibung der Thermodynamik des Systems mit der CALPHAD-Methode kann also nicht zurückgegriffen werden. Deswegen ist ein Rückgriff auf die oben gemachten Annahmen durchaus plausibel. Ein angenommener hypothetischer 
Verlauf der Energiekurven ist in Abbildung 4-19 aufgetragen. Auf der Al-reichen Seite zeigen beide Kurven ein Entmischungsverhalten, mit einem sehr ähnlichen Verlauf, also auch ähnlicher Stabilität, innerhalb der Mischungslücke.

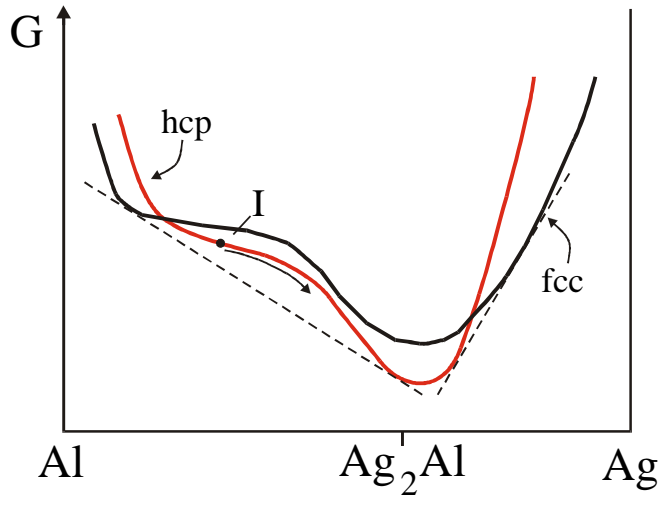

Abbildung 4-19: Vorgeschlagener hypothetischer Verlauf der Freien Energie Kurven.

Gegen eine höhere Stabilität der hcp- gegenüber der fcc-Phase gibt es auch für Al-reiche Zusammensetzungen keinen physikalischen Widerspruch. Demnach können sehr wohl treibende Kräfte zur Bildung der hcp-Phase weit weg vom Gleichgewicht wirken. Symbolhaft ist dies an der Stelle I in Abbildung 4-19 eingezeichnet. In späteren Zuständen nimmt die Reaktionsrate ab und die Phase entmischt, wie der $\chi^{2}$-Test in Abbildung 4-11 für die bei $250^{\circ} \mathrm{C}$ ausgelagerten Probe deutlich macht. Unter Umständen geschieht dies sogar über einen spinodalen Mechanismus. Die Entmischung führt jedoch nicht zu einem heterogenen Gefüge, da gleichzeitig die Gleichgewichtskonzentration $\mathrm{Ag}_{2} \mathrm{Al}$ durch langsame Volumendiffusion eingestellt wird (Pfeil entlang der hcp-Kurve).

In der bisherigen Diskussion ist die Charakterisierung der Produktphase als "metastabil" bewußt vermieden worden, da nicht mit Sicherheit beurteilt werden kann, ob die Phase wirklich metastabile Eigenschaft hat, die aus einem lokalen Minimum der Freien Energie folgen würde oder ob ein stationärer Zustand durch sich ausgleichende Diffusionsströme vorliegt. Die homogene Struktur der intermediären Phase bei $140^{\circ} \mathrm{C}$ (vgl. Abbildung 4-10c) deutet auf einen metastabilen Charakter hin. Dem widerspricht allerdings die kontinuierliche Umwandlung in den Gleichgewichtszustand.

Es wird deutlich, daß die Frage, warum sich die hexagonale Gleichgewichtsphase $\gamma$ weit weg von ihrer Gleichgewichtskonzentration bewiesenermaßen als Vorläufer der eigentlichen $\gamma$-Phase $\mathrm{Ag}_{2} \mathrm{Al}$ zeigt, ohne entsprechende thermodynamische Daten, wie CALPHAD-Rechnungen, die auch experimentelle Daten zu Nichtgleichgewichtszuständen berücksichtigen, nicht befriedigend beantwortet werden kann.

\subsubsection{Kinetische Stabilisierung der Vorläuferphase}

Wie sich zeigt, deckt eine neue Methode auch neue grundlegende Fragen auf. Eine der interessantesten ist die Reaktionsasymmetrie zwischen $\mathrm{Ag}$ - und Al-Schicht. Die Interreaktion findet bevorzugt in der Ag-Lage statt, unter Bildung einer Al-reichen 
Vorläuferphase $\mathrm{Al}_{2} \mathrm{Ag}$ bei schnellem Antransport der benötigten $\mathrm{Al}-\mathrm{Atome}$. Für die Betrachtung der Kinetik sind die benötigten Volumendiffusionsdaten aus Tabelle 3 in Abbildung 4-20 auf unseren Temperaturbereich extrapoliert. Augenfällig ist der große Unterschied in den Beweglichkeiten innerhalb der Al- und der Ag-Schicht. Dies ist aber verständlich, da in der Regel der Diffusionskoeffizient mit der Schmelztemperatur korreliert ist. In unserem Fall bedeutet das einen höheren Diffusionskoeffizienten für $\mathrm{Al}$, der sich zudem noch als weitgehend unabhängig von der Konzentration erweist. Auf der anderen Seite zeigen die Diffusionsdaten für Ag eine deutliche Abhängigkeit vom Al-Gehalt [LANDOLt, ADDA]. Die Mobilität steigt klar mit zunehmenden Al-Gehalt an. Leider existieren keine Daten über die Konzentrationsabhängigkeit der Diffusion in der hexagonalen $\gamma$-Phase. Man kann aber vermuten, daß ähnliche Abhängigkeiten wie in der fcc-Struktur gelten, da die nächste Nachbar-Umgebung ähnlich ist. Demnach würde ein hoher Al-Anteil gleichbedeutend mit einer großen Beweglichkeit innerhalb der $\gamma$-Phase sein.

Wie ist aber nun zu verstehen, daß diese Konzentration nicht fluktuiert, sondern sich über einen ausgedehnten Temperatur- und Ortsbereich stabil verhält? Eine erste Antwort auf diese Frage gibt die von WALSER und BENÉ entwickelte Regel zur Beschreibung der Phasenbildung bei Festkörperreaktionen [WALSER, BENÉ]. Sie besagt, daß sich zuerst diejenige kongruent schmelzende Phase bildet, die am nächsten zum tiefsten Eutektikum des Gleichgewichtphasendiagramms liegt, da an diesem Punkt die größte Mobilität besteht und es zu einer effektiven Durchmischung kommen kann. Die Regel wurde allerdings für Metall/Silizid-Reaktionen aufgestellt und läßt sich nur unter Vorbehalt auf Metall/Metall-Systeme übertragen. In einer Weiterführung geht BENÉ von einem Prinzip der maximalen Reduktionsrate der Freien Energie aus. Angewandt bedeutet dies, daß die Al-reiche Phase mit hcp-Struktur auch weit weg vom Gleichgewicht stabilisiert wird, da bei dieser Zusammensetzung ein beschleunigter Transport stattfinden kann und somit die Reaktionsrate extrem ansteigt. Bedenkt man, daß die intermediären Phase in der Tat am eutektischen Punkt des Systems befindet, so ist die Regel von PRETORIUS in diesem Zusammenhang von Bedeutung [Pretorius1, Pretorius2]. Er erweitert den Gedankengang von WALSER und BENÉ um eine thermodynamische Komponente und führt das Konzept der effektiven Bildungsenthalpie ein. Die bei der Interreaktion erwartete Produktphase ist die mit der negativsten Bildungsenthalpie am eutektischen Punkt. Dies vereint zum einen die Forderung nach maximaler Reduktionsrate der Freien Energie als auch die Erwartung der eutektischen Zusammensetzung an der Grenzfläche zwischen den beiden Reaktanden aufgrund der hohen Mobilität. Die vorher geforderte kongruente Grenzfläche ist bei diesem Konzept nicht mehr erforderlich. Diese Regel läßt sich erfolgreich auf viele Systeme anwenden, wobei es auch Ausnahmen von diesem Prinzip gibt.

Eine weitere neu aufgeworfene Frage ist die in den Untersuchungen gefundene Asymmetrie der Reaktionskinetik in $\mathrm{Ag}-$ und $\mathrm{Al}-\mathrm{Schicht}$. In den Untersuchungen von BAGLIN bestätigt sich zudem eindeutig eine schnelle Korngrenzendiffusion innerhalb des Al [BAGLIN]. Warum findet dann die Interreaktion bevorzugt in der Ag-Lage statt? Dies liegt an der bereits beschriebenen direkten Bildung der $\gamma$-Phase 
in ihrer Gleichgewichtskonzentration in den Korngrenzen der Al-Schicht. Gemäß unseres obigen Analogieschlusses ist die Mobilität mit zunehmendem Ag-Gehalt extrem behindert, so daß kein schneller Transport des benötigten Al durch die "versiegelten" Korngrenzen möglich ist, - unabhängig davon, ob die gebildete Phase nun fcc- oder hcp-Struktur hat.

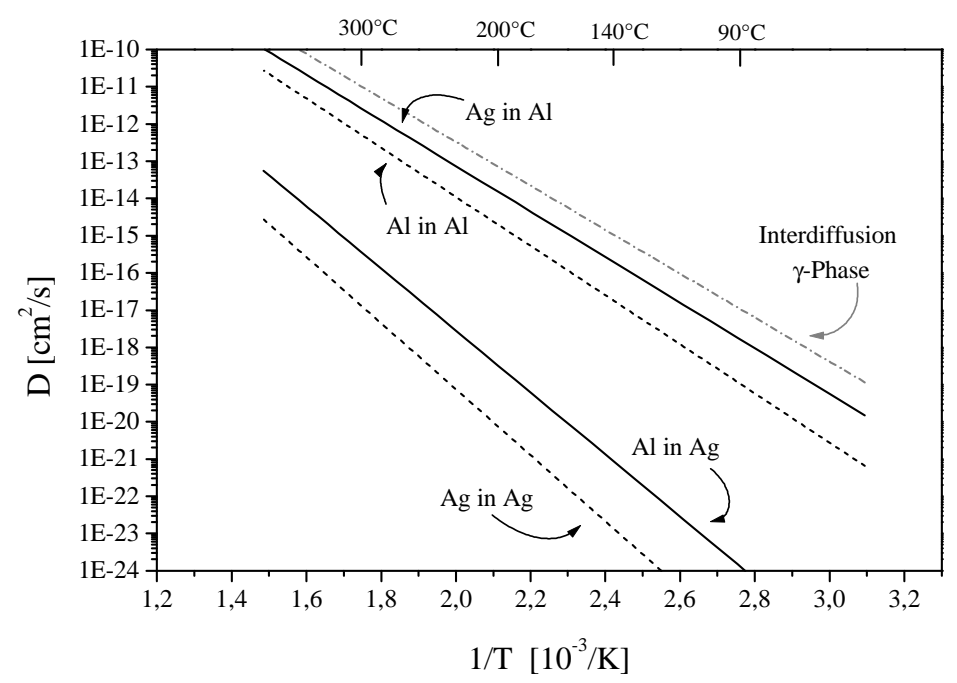

Abbildung 4-20: Gegenüberstellung der Volumendiffusionsdaten

\subsubsection{Modellvorstellung des Reaktionsmechanismus}

Auf Basis der bisher diskutierten Erkenntnisse läßt sich nun folgende Vorstellung des Reaktionsverlaufes aufstellen, skizziert in Abbildung 4-21. Die Frühstadien der Interreaktion sind dank der hochauflösenden Analysemethode eindeutig charakterisierbar. Sie sind geprägt durch schnelle Korngrenzendiffusion des Al innerhalb der Ag-Lage bis in die Tiefe der Schicht. Im nächsten Schritt werden die Ag-Körner von einer 2-3 nm breiten Al-reichen Segregationszone umschlossen. Innerhalb der Korngrenzen und bevorzugt in deren Tripelpunkten wird die Bildung der hexagonalen $\gamma$-Phase beobachtet. Dieses Ergebnis steht in Übereinstimmung mit den Untersuchungen an übersättigten $\mathrm{AlAg}$-Legierungen [NichOLSEN]. Abweichend von den Legierungsanalysen wird die $\boldsymbol{\gamma}$-Phase nicht in ihrer erwarteten stöchiometrischen Zusammensetzung von $\mathrm{Ag}_{2} \mathrm{Al}$ stabilisiert, sondern als $\mathrm{Al}$-reiche Phase mit einem Gehalt von 67 at.-\% Al. Die wahrscheinlichste Ursache für diesen eindeutig nachgewiesenen Effekt liegt in der erhöhten Mobilität der Al-reichen Phase, wie bereits in Abschnitt 4.4.4 diskutiert wurde. Eine hohe Mobilität bedeutet einen schnellen Ablauf der Reaktion und somit eine hohe Reduktionsrate der Freien Energie. 

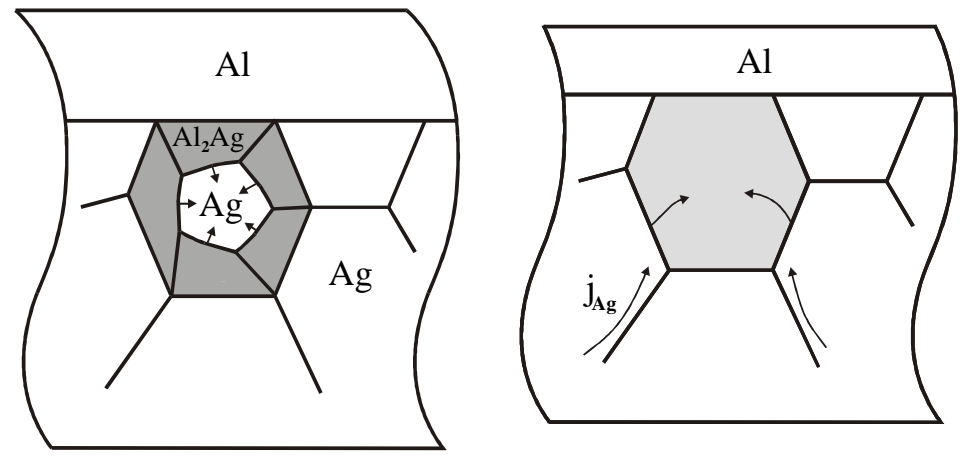

\begin{abstract}
Abbildung 4-21:Schematische Darstellung des Reaktionsmechanismus an einem Korn im Detail vorgestellt: a) Im ersten Schritt bildet sich eine Al-reiche Phase in Form einer Schale um die Ag-Körner (grau markiert in a.). b) Nach Aufzehrung der Ag-Körner erfolgt die Einstellung der Gleichgewichtskonzentration in der $\mathbf{A l}_{2} \mathbf{A g}-$ Phase durch Volumendiffusion.
\end{abstract}

Nachdem die Ag-Lage verbraucht ist, erfolgt die Einstellung der Gleichgewichtskonzentration durch Volumendiffusion in der intermediären Phase. Aufgrund des steigenden $\mathrm{Ag}$-Gehaltes nimmt die Diffusivität innerhalb der hexagonalen Phase stetig ab. Wie die Röntgendaten (vgl. Abbildung 4-17) zeigen, kommt die Umwandlung erst nach sehr langen Zeiten oder bei Erhöhung der Temperatur zum Ende.

Dieser vorgestellte Ablauf ist dem von BAGLIN formulierten Schema sehr ähnlich [BAGLIN]. Auch dort werden die Ag-Korngrenzen als Ort der Interreaktion bestimmt und die bevorzugte Bildung der Produktphase an Tripelpunkten formuliert. Bei den aufgenommenen RBS-Spektren wird allerdings angenommen, daß sich die Keimbildung in der Gleichgewichtskonzentration vollzieht. Das dies nicht der Fall ist, zeigen signifikant und in mehreren Messungen bestätigt die Analysen der Tomographischen Atomsonde. Bei genauerer Betrachtung widersprechen die in der Literatur zitierten Ergebnisse nicht den hier vorgestellten Untersuchungen. Unter Einbezug der dreidimensionalen Reaktionsmorphologie müssen aufgenommene RBS-Spektren in diesen Verlauf münden, ungeachtet der auf Nanometerskala vorliegenden Reaktionszonen.

Unter Berücksichtigung des beschriebenen Reaktionsmechanismus, müssen die bei DSC-Messungen auftretenden exothermen Doppelpeaks anders gedeutet werden. RoY interpretiert die bei $\mathrm{Ag} / \mathrm{Al}$ auftretenden Doppelpeaks als Kennzeichen für Keimbildung und Wachstum der Produktphase [RoY]. Wahrscheinlicher ist eine Zuordnung des ersten Peaks bei $160^{\circ} \mathrm{C}$ bei einer Heizrate von $20 \mathrm{~K} / \mathrm{min}$ der Ausbildung der intermediären Phase $\gamma^{*}$, also der Formierung der hexagonalen Produktphase weg vom Gleichgewicht. Die spätere Umlösung in die Gleichgewichtskonzentration und gleichzeitiges Dickenwachstum resultiert in der zweiten Wärmetönung bei $190^{\circ} \mathrm{C}$. Die von RoY bestimmte Größe eines Keimes von $50 \mathrm{~nm}$ ist daher auf die Anwendung eines falschen Modells zurückzuführen.

Die so gewonnenen Erkenntnisse sind grundlegend für das physikalische Verständnis der Frühstadien bei Interreaktionen. Bei dem benachbarten System Al/Ni existieren ähnliche Fragestellungen. In den Frühstadien der Schichtreaktion findet MicHAELSEN 
eine metastabile Vorläuferphase vor Bildung der ersten Gleichgewichtsphase $\mathrm{NiAl}_{3}$ [MICHAELSEN1, BARMAK]. Interessanterweise zeigt die Vorläuferphase in Elektronenbeugungsexperimenten eine B2-Struktur auf. Die einzige intermetallische Phase des Systems mit dieser Gitterstruktur ist die NiAl (B2)-Phase. Allerdings liegt nach dem Gleichgewichtsphasendiagramm die Konzentration des gefundenen Vorläufers mit 63 at.-\% Al außerhalb des Stabilitätsbereiches der NiAl-Phase. Einzig logische Schlußfolgerung ist nach MiCHAELSEN, daß sich $\mathrm{zu}$ Anfang eine metastabile B2-NiAl Phase mit 63 at.-\% Al bildet. Die Interpretation der kinetischen Stabilisierung einer Nicht-Gleichgewichtskonzentration mit Struktur der eigentlichen Produktphase ist also durchaus plausibel.

\subsection{Zusammenfassung der Ergebnisse zum System Ag/Al}

Die TAP-Untersuchungen haben gezeigt, daß sich der Reaktionsmechanismus wesentlich komplexer darstellt als das bisher postulierte einfache Lagenwachstum einer Gleichgewichtsphase. Die Reaktionskinetik wird bestimmt durch die mikrostrukturellen Eigenschaften des Systems. Eine quantitative Beschreibung der in XRD-Messungen bestimmten Kinetik ist aufgrund der komplexen Reaktionsmorphologie bisher nicht gelungen. Für eingehendere Betrachtungen der Phasenstabilität im Al-reichen Konzentrationsbereich sind thermodynamische Rechnungen wie nach der CALPHAD-Methode notwendig. Bisher vorliegende Daten sind nicht für die Modellierung der Freien Energien geeignet, so daß ein vermutlicher Kurvenverlauf vorgeschlagen wurde.

Der Verlauf der Ag/Al-Interreaktion läßt sich in folgende Stufen unterteilen:

- $\quad$ Bei niedrigen Auslagerungstemperaturen und kurzen Zeiten verläuft die Reaktion gemäß planarer Modellvorstellungen. Bei weiteren Auslagerungsschritten entwickelt sich aufgrund des nanokristallinen Gefüges eine komplizierte 3D-Reaktionsmorphologie. Eine Beschreibung als planare Schichtreaktion ist nicht mehr anwendbar.

- Die Reaktion wird getrieben durch Al-Eindiffusion in die Korngrenzen der Ag-Schicht. Dort bildet sie Al-reiche Schalen um die 5 bis $10 \mathrm{~nm}$ großen Körner mit einer Konzentration von bis zu 60 at.-\% Al. Die hexagonale Phase bildet sich bevorzugt in Tripelpunkten der Korngrenzen und wächst unter Aufzehrung der Ag-Körner.

- Interessanterweise bildet sich die erwartete hdp-Gleichgewichtsphase $\gamma$ weit weg vom Gleichgewicht mit einer Stöchiometrie von $\mathrm{Al}_{2} \mathrm{Ag}$. Vermutliche Ursache dafür ist die schnelle Reduktion der Freien Energie durch die größere Mobilität in Al-reichen Phasen.

Ist die restliche $\mathrm{Ag}-\mathrm{Schich}$ verbraucht, so erfolgt die langsamere Einstellung der Gleichgewichtskonzentration. 
- Innerhalb der Al-Korngrenzen bildet sich die Gleichgewichtsphase $\gamma$ direkt in ihrer Gleichgewichtskonzentration. Allerdings ist der gebildete Anteil verschwindend gering aufgrund der niedrigen Mobilität innerhalb der $\gamma$-Phase mit ihrer Stöchiometrie von $\mathrm{Ag}_{2} \mathrm{Al}$. 


\section{Nanoanalytik von $\mathrm{Co} / \mathrm{Cu} / \mathbf{P y}-$ Schichtsystemen}

\subsection{Vorbemerkungen}

Im Jahr 1986 wurde von GRÜNBERG durch Licht-Streuexperimente an $\mathrm{Fe} / \mathrm{Cr}-$ Multilagenschichten die Existenz einer Austauschkopplung zwischen ferromagnetischen Lagen über eine metallische Zwischenschicht hinweg nachgewiesen [GRÜNBERG]. Im darauffolgenden Jahr wurde diese Wechselwirkung von einer anderen Gruppe ebenfalls am System $\mathrm{Fe} / \mathrm{Cr}$ mit Hilfe von Spinpolarisierter Elektronenstreuung bestätigt [CARBONE].

Das Interesse an diesen Schichtsystemen wuchs schlagartig, als 1988 und 1989 bekannt wurde, daß ihr elektrischer Widerstand durch Anlegen eines magnetischen Feldes drastisch sinkt [BAIBICH, BINASCH]. Wegen der Größe des Effektes, bei 4,2 K etwa $50 \%$, wurde er als GiantMagnetoResistance (GMR) bezeichnet. Diese Bezeichnung dient heute vor allem zur Kennzeichnung des Effektes, auch wenn die Widerstandsänderung zum Teil nur $0,1 \%$ beträgt.

Inzwischen sind an vielen anderen Schichtsystemen ähnliche Magnetfeldabhängigkeiten des Widerstandes gefunden worden, aber auch in granularen $\mathrm{CuCo}-$ Systemen [BERKOwITZ], in sogenannten Spin-Ventilen ("spin valves") [DIENY] und in granularen Multilagen [LuO].

Die Ursache des veränderbaren elektrischen Widerstandes durch Variation des Magnetfeldes liegt in der spinabhängigen Streuung der Leitungselektronen. Abhängig von der relativen Ausrichtung der magnetischen Momente in den ferromagnetischen Lagen und von dem sie trennenden Metall, der Zwischenlage, folgt eine Streuung der Leitungselektronen, die von der Orientierung ihrer Spins abhängt. Die Streuung kann dabei im ferromagnetischen Material oder an der Grenzfläche zur Zwischenschicht stattfinden. Von Bedeutung sind die durch die Austauschaufspaltung unterschiedlichen Zustandsdichten der 3d-Bänder des ferromagnetischen Materials für spin-up und für spin-down Elektronen an der Fermikante, die die Zustände bereitstellen, in die Leitungselektronen gestreut werden. Diese Asymmetrie ist bei starken Ferromagneten, wie $\mathrm{Ni}$ und $\mathrm{Co}$, ausgeprägter, als bei schwachen. Insbesondere ändert sie sich mit Zulegierung einer anderen Komponente. Sind die magnetischen Momente zweier ferromagnetischer Lagen antiparallel zueinander ausgerichtet, so sind die Majoritätselektronen der einen Lage die Minoritätsladungsträger der anderen. Bewegt das Elektron sich also aus dem ersten Bereich, in dem es wenig gestreut wird, in den zweiten, so ist dort die Streuwahrscheinlichkeit wesentlich höher. Umgekehrt verhält es sich natürlich mit den Majoritätsladungsträgern der anderen Lage, so daß im Mittel deren Widerstände $\rho_{\mathrm{afm}}$ identisch sind. Werden allerdings die magnetischen Momente der ferromagnetischen Lagen durch ein äußeres Feld parallel ausgerichtet, so ist für eine Ladungsträgerkomponente der elektrische Widerstand $\rho_{\mathrm{fm}}$ innerhalb der ganzen Probe gesenkt. 
Die resultierende GMR-Effektamplitude berechnet sich dann aus ${ }^{10}$ :

$$
G M R=\frac{\rho_{a f m}-\rho_{f m}}{\rho_{f m}} .
$$

Die meisten Arbeiten zur Untersuchung der Kopplung gehen von der RUDERMANNKITTEL-KASUYA-YOSIDA (RKKY)-Kopplung aus. Faßt man die einzelnen Atome des magnetischen Materials, die sich direkt an der Grenzfläche befinden, als Fremdatome im nicht-magnetischen Material der Zwischenschicht auf, so führt jedes im Sinne der RKKY-Theorie zu einer Polarisation der Leitungselektronen dieser Zwischenschicht. Überlagert man alle diese Oszillationen, so interferieren beinahe alle destruktiv. Diejenigen, die letztlich zur Kopplung führen, sind dadurch charakterisiert, daß ihre Oszillationsperioden mit bestimmten Vektoren $\mathrm{q}_{\mathrm{i}}$ zwischen den Fermiflächen des Kopplungsmaterials in Verbindung gebracht werden können, wobei diese in Richtung der Schichtnormalen zeigen. Je nach Wachstumsrichtung der Schichten resultieren dann verschiedene Oszillationsperioden $\lambda_{i}=\left(2 \pi / q_{i}\right)$. Die Abhängigkeit der Kopplung von der Bandstruktur des Zwischengittermaterials folgt direkt aus dem Experiment von Bово [Вово]. Durch Zulegieren von Ni zur $\mathrm{Cu}$-Zwischenschicht konnte eine unmittelbare Auswirkung auf die Kopplungsperiode nachgewiesen werden.

Die schnelle Erforschung der Eigenschaften des Magnetowiderstandes begründet sich auch durch das große Interesse der Industrie am Einsatz von GMR-Sensoren. Diese kommen zum Beispiel als Drehzahlmesser im Automobilbau oder als Winkeldetektor an Stellknöpfen von u.a. Waschmaschinen zum Einsatz. Darüber hinaus ist die Elektronikindustrie an der Entwicklung von magnetischen Speicherbausteinen, MRAM, interessiert.

Der Nachteil der zuvor erwähnten Systeme $\mathrm{Fe} / \mathrm{Cr}$ und $\mathrm{Co} / \mathrm{Cu}$ liegt in den hohen benötigten magnetischen Feldern zur Überwindung der antiferromagnetischen Kopplung. Das war lange Zeit der limitierende Faktor in der industriellen Anwendung dieser Vielfachschichten. Außerdem ist man in der Regel weniger an hohen Koerzitivfeldstärken als an hoher Ansprechempfindlichkeit auf Magnetfeldänderungen interessiert. Deshalb wurden andere weichmagnetische Materialien auf ihre Anwendungstauglichkeit untersucht. Ein vielversprechender Kandidat ist das Permalloy, eine Ni-Fe Legierung in dem Verhältnis 81:19, aufgrund der geringen Koerzitivfeldstärke von wenigen $\mathrm{Oe}$. Mit Schichtsystemen bestehend aus $\mathrm{Cu} / \mathrm{Py}-$ oder Au/Py-Mulitlagen wurden schon vernünftige GMR-Werte mit hervorragender Empfindlichkeit realisiert [HEITMANN2, PARKIN].

Die antiferromagnetische Kopplung ist nicht Voraussetzung zur Erreichung des GMR-Effektes. In weiteren vorgeschlagenen Anwendungssystemen, sogenannten Trilayer-Systemen oder Spin-valves liegt die Dicke der entkoppelnden

\footnotetext{
${ }^{10}$ In der Literatur wird bei Untersuchungen granularer Systeme zur Berechnung des GMR-Effektes die Gleichung $G M R=\frac{\rho_{a f m}-\rho_{f m}}{\rho_{a f m}}$ benutzt und bei Lagensystemen die Formel (5.1).
} 
Zwischenschicht außerhalb der Kopplungsperiode [LotTis, Hoscoe]. Dennoch lassen sich die magnetischen Momente parallel oder antiparallel durch ein äußeres Magnetfeld ausrichten. Das erreicht man durch geschickte Wahl der beteiligten Schichten. Ein aktuelles Anwendungssystem ist die Kombination aus hartmagnetischer Co-Lage und weichmagnetischer Py-Schicht, die durch eine dickere $\mathrm{Cu}-\mathrm{Z}$ wischenschicht voneinander entkoppelt sind. Mit geringen äußeren Magnetfeldstärken läßt sich das magnetische Moment der Py-Lage beliebig drehen, ohne daß bereits die Co-Lage ihre Magnetisierungsrichtung ändern würde. Der Winkel zwischen den beiden unterschiedlichen Magnetisierungsrichtungen verursacht dann einen entsprechenden Verlauf des Magnetowiderstandswertes.

Das nährt die Vorstellung, durch Kombination der beiden Materialien in den jeweils passenden Dicken für jede anwendungsgeforderte Widerstandskennlinie die richtigen Systeme maßschneidern zu können [HwANG].

In der technischen Anwendung ist die Temperaturbeständigkeit eine wichtige Eigenschaft. Untersuchungen an gekoppelten $\mathrm{Cu} / \mathrm{Co}$ und $\mathrm{Cu} / \mathrm{Fe}_{19} \mathrm{Ni}_{81}$ Multilagen haben gezeigt, daß bei Wärmebehandlungen bis $350^{\circ} \mathrm{C}$ [RÄTZKE, MAO], bzw. bei $\mathrm{Cu} / \mathrm{Fe}_{19} \mathrm{Ni}_{81}$ bis $250^{\circ} \mathrm{C}$ [HWAng, HeITMANN2], eine deutliche Steigerung des GMR-Effektes $\mathrm{zu}$ erreichen ist. Die allgemeine herrschende Auffassung der aktuellen Literatur interpretiert das Ansteigen der GMR-Amplitude als Folge der Reduktion von Grenzflächenrauhigkeiten. Beim Überschreiten dieser Auslagerungstemperatur nimmt der Magnetowiderstand wieder ab. Verantwortlich dafür wird die $\mathrm{Cu}$-Eindiffusion in die $\mathrm{Co}$-Korngrenzen gemacht, die ein Entkoppeln der einzelnen Co-Körner der Lage und damit ein Zusammenbrechen des GMR-Effektes verursachen soll [RÄTZKE].

Neben der geometrischen Schärfe der Grenzflächen hat ihre chemische Zusammensetzung einen großen Einfluß auf den GMR-Effekt. Kürzlich veröffentlichte ab-initio Berechnungen von MERTIG und ZAHN sagen vorher, daß das Hinzulegieren von $\mathrm{Fe}$ in die $\mathrm{Co}-\mathrm{Grenzlage}$ in einem $\mathrm{Co} / \mathrm{Cu}-\mathrm{Schich}$ tpaket den GMR-Effekt aufgrund einer erhöhten Spinanisotropie deutlich erhöhen würde [ZAHN1, ZAHN2, MERTIG]. In einem von HWANG durchgeführten Experiment wurde eine 0,5 Å dicke Fe-Lage in die Grenzflächen eines $\mathrm{Cu} / \mathrm{Co}-$ Multilagenpaketes eingefügt [HwANG]. Interessanterweise zeigte sich eine erhöhte thermische Stabilität und eine deutliche Zunahme der GMR-Amplitude. Ein ähnlicher Effekt wurde beim Einfügen einer $3 \AA$ dicken $\mathrm{Co}-$ Schicht zwischen die Lagen eines $\mathrm{Cu} / \mathrm{Py}-\mathrm{Multilagensystems}$ beobachtet.

Die zuvor vorgestellten Experimente und Rechnungen machen klar, daß die GMR-Effektamplitude extrem sensibel von den strukturellen und chemischen Eigenschaften des Systems bis hinunter zu Monolagenveränderungen abhängt.

Ziel der bisherigen Arbeiten war es, bei geeigneter Auswahl der Schichtmaterialien und Geometrie der Proben (Dicke der einzelnen Lagen, Stapelfolge,...) die Beiträge der magnetischen Austauschwechselwirkung (bilinear und/oder biquadratisch) zu identifizieren und die GMR-Effektamplitude als Funktion der Bandstruktur, der Ummagnetisierungs- und der Temperaturabhängigkeit zu verstehen [HEINRICH]. 
Für metallische Schichten, die im allgemeinen nicht epitaktisch sondern nanokristallin aufwachsen, ist jedoch zu erwarten, daß die GMR-Effektamplitude ganz wesentlich auch eine Funktion der Mikrostruktur, d.h. räumliche Verteilung der Phasen/Kornstruktur ist. Aufgrund der bei koppelnden Systemen notwendigen geringen Dicke (üblicherweise $<3 \mathrm{~nm}$ ) der nicht-ferromagnetischen Trennschichten, ist die mikrostrukturelle und chemische Charakterisierung der Grenzflächen und der angrenzenden Phasen nur noch mit wenigen hochentwickelten mikroskopischen Techniken möglich. Daher sind in diesem Zusammenhang eine ganze Reihe von physikalischen Grundlagenfragen bisher ungeklärt geblieben: $\mathrm{Ab}$ welcher Schichtdicke und unter welchen Bedingungen sind Schichtdurchbrüche, sog. pinholes, von Bedeutung und wie ist ihr Einfluß auf den Magnetowiderstand? Gerade bei Anwendungen im erhöhten Temperaturbereich ist die Frage nach der Langzeitstabilität der Systeme bei auftretender Interreaktion nicht zu vernachlässigen. Theoretische Betrachtungen vermuten eine Erhöhung des GMR-Effektes durch Segregation bestimmter Fremdatome an den Grenzflächen [Mertig, Zahn1, Zahn2]. Auch zu diesen Überlegungen gibt es keinen direkten experimentellen Nachweis.

Es ist einsichtig, daß die Aufklärung dieser Fragen notwendig ist, um anwendungsreife Systeme zu entwickeln und weiter zu verbessern. An dieser Stelle setzt das Potential der Tomographischen Atomsonde an. Sie ermöglicht die Analyse der Atomverteilung in einem 3D-Probenvolumen einer üblichen Größe von $10 \cdot 10 \cdot 100 \mathrm{~nm}^{3}$. Diese atomare Auflösung ist bisher von keiner anderen Methode erreicht [Blavette1, BlavetTE2]. Die im folgenden vorgestellten TAP-Analysen bilden deshalb den Schwerpunkt der Charakterisierung der präparierten magnetischen Schichtsysteme.

\subsection{Experimentelle Vorarbeiten}

Zur genaueren Charakterisierung der Temperaturstabilität des magnetischen Widerstandes wurden planare $\mathrm{Co} / \mathrm{Cu} / \mathrm{Py}-$ Multilagen wärmebehandelt unter permanenter Aufzeichnung von Röntgendiffraktogrammen. Die Proben mit einem Schichtaufbau von $\left[\mathrm{Co}_{4} \mathrm{~nm} / \mathrm{Cu}_{4 \mathrm{~nm}} /\left(\mathrm{Fe}_{19} \mathrm{Ni}_{81}\right)_{4 \mathrm{~nm}} / \mathrm{Co}_{4} \mathrm{~nm}\right]_{40}$ wurden in der Bielefelder Gruppe von Prof. Reiss hergestellt (vgl. Abschnitt 3.3). In Abbildung 5-1 sind einige charakteristische Stadien der Mikrostrukturveränderung während des Auslagerungsvorganges dargestellt. Das Schichtpaket weist im as-sputtered Zustand eine starke (111)-Textur auf. Es sind nur geringe Anteile von (200)-Orientierungen im Röntgendiffraktogramm vorhanden. Aufgrund der geringen Einzellagendicke von $4 \mathrm{~nm}$ erscheint der (111)-Reflex nicht an der Stelle der Reinkomponenten, sondern dazwischen unter Bildung von Satellitenreflexen auf der rechten und linken Seite. Am Schichtsystem $\mathrm{Co} / \mathrm{Cu}$ hat HAMP bereits gezeigt, daß Einzellagenreflexe nur bei Schichtdicken oberhalb von $10 \mathrm{~nm}$ ausgebildet sind und darunter deutlich der mittlere Reflex dominiert [HAMP]. Den Diffraktogrammen nach zu urteilen, hat die Co-Schicht ebenfalls eindeutig fcc-Struktur. Dies ist nicht verwunderlich, da in 
weiterführenden Experimenten bis $\mathrm{zu}$ einer Co-Lagendicke von $5 \mathrm{~nm}$ keine Strukturumwandlung in hcp festgestellt wurde [HüTTEN].
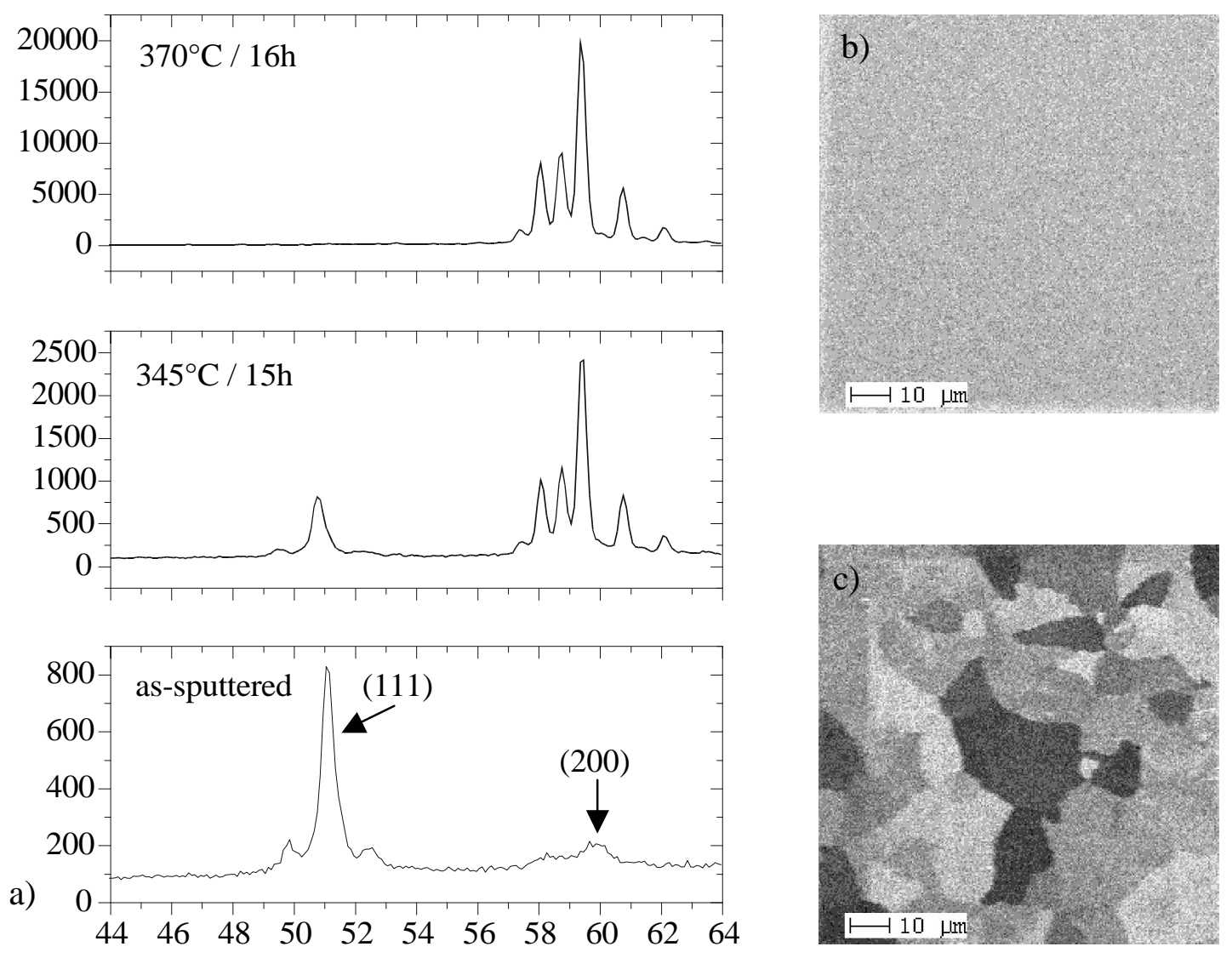

Abbildung 5-1: a) Röntgendiffraktogramme aufgenommen während einer Auslagerung des $[\mathrm{Co} / \mathrm{Cu} / \mathrm{Py} / \mathrm{Co}]_{40}$ - Schichtpaketes. b) Sekundärelektronenbild der Probenoberfläche des Ausgangszustandes. c) Sekundärelektronenbild der Probenoberfläche nach Wärmebehandlung.

Mittels einer Rampenprogrammierung wurde die Auslagerungstemperatur kontinuierlich mit einer Steigung von $20^{\circ} \mathrm{C} / \mathrm{h}$ angehoben. Ab einer Temperatur von $340^{\circ} \mathrm{C}$ reduziert sich die Intensität des (111)-Reflexes bei gleichzeitiger Ausbildung einer Reihe von Satellitenreflexen in (200)-Orientierung. Bereits bei $370^{\circ} \mathrm{C}$ sind nur noch Satelliten um den (200)-Strukturreflex zu detektieren. Das Schichtsystem scheint vollkommen aus der (111)-Struktur kommend in eine (100)-Textur rekristallisiert $\mathrm{zu}$ sein. Eine Umordnung der Gitterstruktur in Schichtnormalenrichtung hat immense Konsequenzen auf die Kopplungseigenschaften des Multilayers. Aufgrund der Tatsache, daß (111)- und (100)-Richtungen deutlich unterschiedliche Kopplungslängen besitzen $\left[(100): \quad \lambda_{1}^{\text {theor. }} \approx 2,6\right.$ und $\lambda_{2}{ }^{\text {theor. }} \approx 5,9$ Monolagen // (111): $\quad \lambda^{\text {theor. }} \approx 4,5$ Monolagen], würde eine vorher gekoppelte Probe ihre GMR- Eigenschaften verlieren, da die paramagnetische $\mathrm{Cu}-$ Zwischenschicht in ihrer Dicke nicht mehr dem jeweiligen Kopplungsmaximum entsprechen würde [BRUNO, JOHNSON1]. Andererseits ließen sich Proben definierter Zwischenschichtdicke herstellen und durch einen Rekristallisationsprozeß ins 
Kopplungsmaximum bringen. Die im TEM bestimmte laterale Korngröße war im assputtered Zustand vergleichbar der Schichtdicke von $4 \mathrm{~nm}$. Sekundärelektronenaufnahmen ${ }^{11}$ der Probe vor und nach der Wärmebehandlung in Abbildung 5-1 b) und c) zeigen ein Anwachsen der lateralen Korngröße auf 20 bis $30 \mu \mathrm{m}$. Ob die Schichtstruktur erhalten geblieben ist und welche chemischen Eigenschaften vorliegen, läßt sich aus den Daten der Röntgendiffraktometrie nicht schließen. Zunächst wurden deshalb Querschnittsproben für die Transmissionselektronenmikroskopie des Ausgangs-zustandes und der in der Röntgenanlage getemperten Probe angefertigt. Abbildung 5-2 a) zeigt ein Hellfeldbild des unbehandelten Zustandes. Es sind deutlich kolumnare Körner in Wachstumsrichtung zu erkennen, wobei keine einzelnen Lagen ausgemacht werden können. Erst die Aufnahme mit kleinem Defokus ermöglicht die Abbildung der einzelnen Schichten. Die Beugungsaufnahme über den in Abbildung 5-2 c) ausgewählten Bereich zeigt körnige Ringstrukturen. Wie aus dem Hellfeldbild schon zu entnehmen ist, ist der as-sputtered Zustand polykristallin.

Ein ganz anderes Bild zeigt die Untersuchung der ausgelagerten Probe. In den Hellfeldbildern in Abbildung 5-2 e)+f) lassen sich keine kolumnaren Strukturen in der Ausprägung wie im Ausgangszustand erkennen. Das steht im Einklang mit den aus den Sekundärelektronenbildern gefolgerten lateralen Korngrößen auf $\mu \mathrm{m}-$ Skala. In dem kleinen Analysebereich des TEM erscheint die Probe daher einkristallin.

Schon im Hellfeldmodus ist die Abfolge der Schichtstruktur selbst ohne Defokus sichtbar. Die Prüfung der vorliegenden Kornorientierung über den ausgewählten Probenbereich gibt in der Tat eine bereits aus den Röntgenuntersuchungen vermutete Würfeltextur wieder. Da keine anderen Reflexe aufgezeigt werden, läßt das nur den Schluß zu, daß das gesamte Schichtsystem in (100)-Orientierung vorliegt.

Eine feinere chemisch sensitive Abbildung läßt sich mit der Z-Kontrastmethode erreichen. Da allerdings die untersuchten Strukturen aus Elementen bestehen, die sich in direkter Nachbarschaft zueinander im Periodensystem befinden, ist Differenzierung der einzelnen Schichten am Rande der Auflösungsgrenze der Methode. Dennoch gibt der chemische Kontrast in Abbildung 5-2 g) den Verlauf der unterschiedlichen Lagen gut aufgelöst wieder und erlaubt aufgrund des Multilayeraufbaus eine direkte Elementzuordnung. Dies ist ein schönes Beispiel für die Leistungsfähigkeit des Z-Kontrastes - Elemente sehr ähnlicher Ordnungszahl auf so kleiner Skala noch auflösen zu können. Sie kann allerdings keine Aussage darüber treffen, ob Diffusionsprozesse auf kleiner Skala stattgefunden haben und die Lagen möglicherweise eine chemisch veränderte Struktur aufweisen. Dies ist der Ansatzpunkt der Tomographischen Atomsonde. Sie ermöglicht eine chemische Analyse auf atomarer Skala.

11 Die Austrittswahrscheinlichkeit der Elektronen ist abhängig von der Kornorientierung und ermöglicht auf diese Weise eine Texturanalyse an der Probenoberfläche. 


\section{$\underline{\text { As-sputtered }}$}

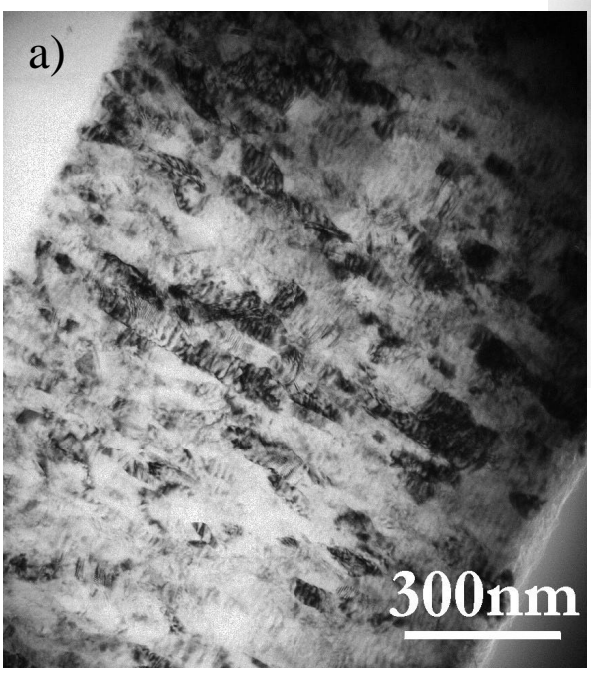

b)

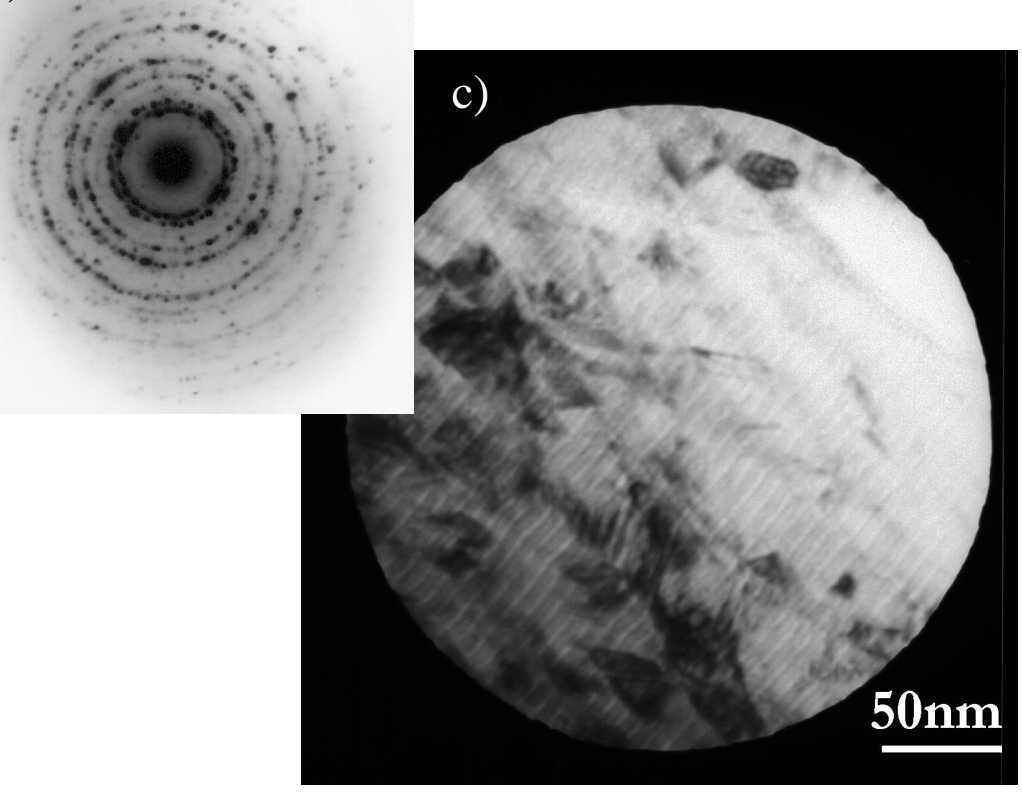

\section{Nach Wärmebehandlung $350^{\circ} \mathrm{C} / 24 \mathrm{~h}$}

e)

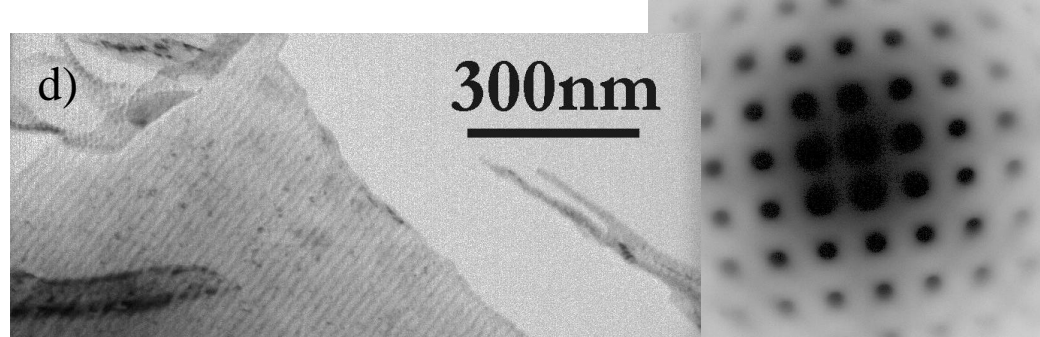

g)

\section{$100 \mathrm{~nm}$}

f)

\section{$100 \mathrm{~nm}$}

\section{Z-Kontrast}

Abbildung 5-2: TEM-Aufnahmen eines querschnittspräparierten [Co/Cu/Py/Co]Schichtpaketes vor und nach der Röntgenheizmessung. a) Hellfeldaufnahme-Übersicht des Ausgangszustandes; b) Beugungsaufnahme des in c) ausgewählten Bereiches; d) Hellfeldaufnahme-Übersicht der wärmebehandelten Probe; e) Beugungsbild des in f) ausgewählten Bereiches; g) Z-Kontrast Aufnahme der ausgelagerten Probe. 


\subsection{Charakterisierung mit Hilfe der Tomographischen Atomsonde}

Schwierigster Punkt bei diesen Untersuchungen ist immer die geeignete Probenpräparation [SChLEIWIES2, Jeske1, Jeske2]. Für die Untersuchungen im TAP wurden zwei verschiedene Präparationswege beschritten. Zum einen wurde die bereits vorgestellte Sputterdeposition durchgeführt. Dafür wurden $\mathrm{Co} / \mathrm{Cu} / \mathrm{Py}-$ Trilayer auf W-Trägerspitzen aufgebracht.

Um der grundsätzlichen Kritik zu begegnen, wonach die Mikrostruktur und deshalb auch der Reaktionsverlauf von planaren Proben und, auf gekrümmte TAP-Spitzen aufgebrachte Schichten, sehr unterschiedlich sein sollen, wurden mit Hilfe der lithographischen Präparation TAP-Proben direkt von planaren Schichtsystemen hergestellt. Über diesen Weg ist ein direkter Vergleich zwischen den beiden unterschiedlichen Probengeometrien möglich.

\subsubsection{Herstellung der Proben}

Die Deposition der $\mathrm{Co} / \mathrm{Cu} / \mathrm{Py}-\mathrm{Schichten}$ auf $\mathrm{W}$-Trägerspitzen erfolgte über den in Abschnitt 3.2 beschriebenen Sputterprozeß. Die zur Herstellung der einzelnen Schichten erforderlichen Parameter der Ar-Ionenquelle und die dabei erreichten Schichtdicken, sind in der nachstehenden Tabelle 4 aufgeführt.

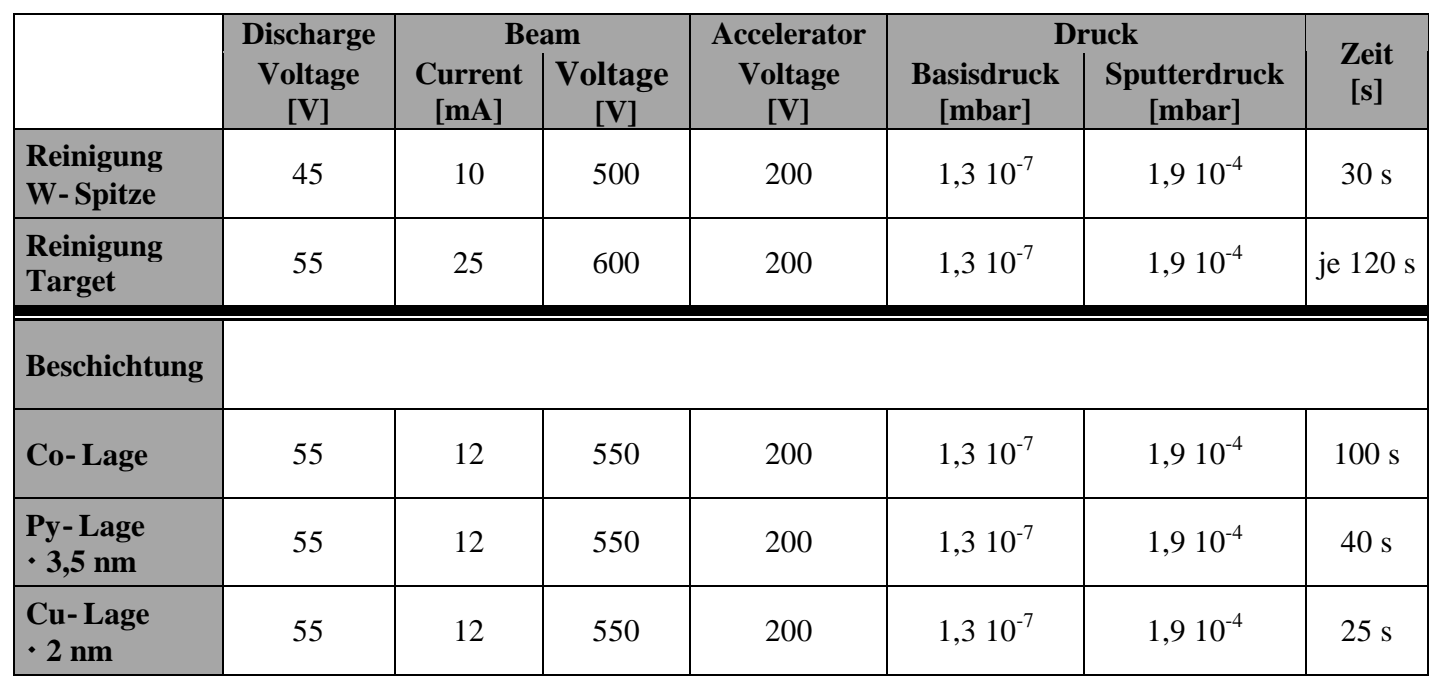

Tabelle 4: Sputterparameter zur Co/Cu/Py-Sputterdeposition

Für die Parameterwahl zur Reinigung der Spitzen im Ionenstrahl gilt das bereits für Ag/Al im Abschnitt 4.3.1 Gesagte. Da zur Beschichtung niedrige Depositionsraten von unter $1 \AA \mathrm{s}$ s verwendet wurden, liegen die erreichten lateralen Körngrößen mindestens im Bereich der Schichtdicke. Ein direkter Nachweis ist mit der Feldionenmikroskopie möglich. Dafür wurde ein Schichtpaket aus $\mathrm{Co}_{6 \mathrm{~nm}} / \mathrm{Cu}_{4} \mathrm{~nm} / \mathrm{Py}_{6 \mathrm{~nm}}$ auf eine W-Spitze aufgebracht und im FIM-Modus während der Feldentwicklung abgebildet. In Abbildung 5-3 a) sind alle drei Lagen zu erkennen. Durch die starke Dekoration des Neon an der Co-Schicht lassen sich im 
äußeren Ring Polstrukturen nur schwer ausmachen. Aus der feldionenmikroskopischen Abbildung läßt sich daher kein Schluß über die Gitterstruktur der gesputterten Co-Schicht ziehen. Die Kornstrukturen und die Lage der gefundenen Pole sind in der Skizze auf der rechten Seite dargestellt. Aufgrund der leicht unterschiedlichen Feldverdampfungsfeldstärken von $\mathrm{Co}$ und $\mathrm{Cu}$ (vgl. Tabelle 5) läßt sich die Oberfläche der $\mathrm{Cu}$-Schicht sauberer abbilden. Ein großer Pol ist deutlich erkennbar, der auf eine größere Kornstruktur im Vergleich zur Co-Schicht hinweist. Innerhalb der zentral erscheinenden Permalloy-Schicht läßt sich wiederum durch die starke Kondensation des Neon und durch den beschränkten Ausschnitt keine Strukturzuordnung treffen. Erst nachdem die Permalloy-Schicht komplett über den gesamten Kanalplattenbereich entwickelt ist, lassen sich feinere Strukturen erkennen (vgl. Abbildung 5-3 b).
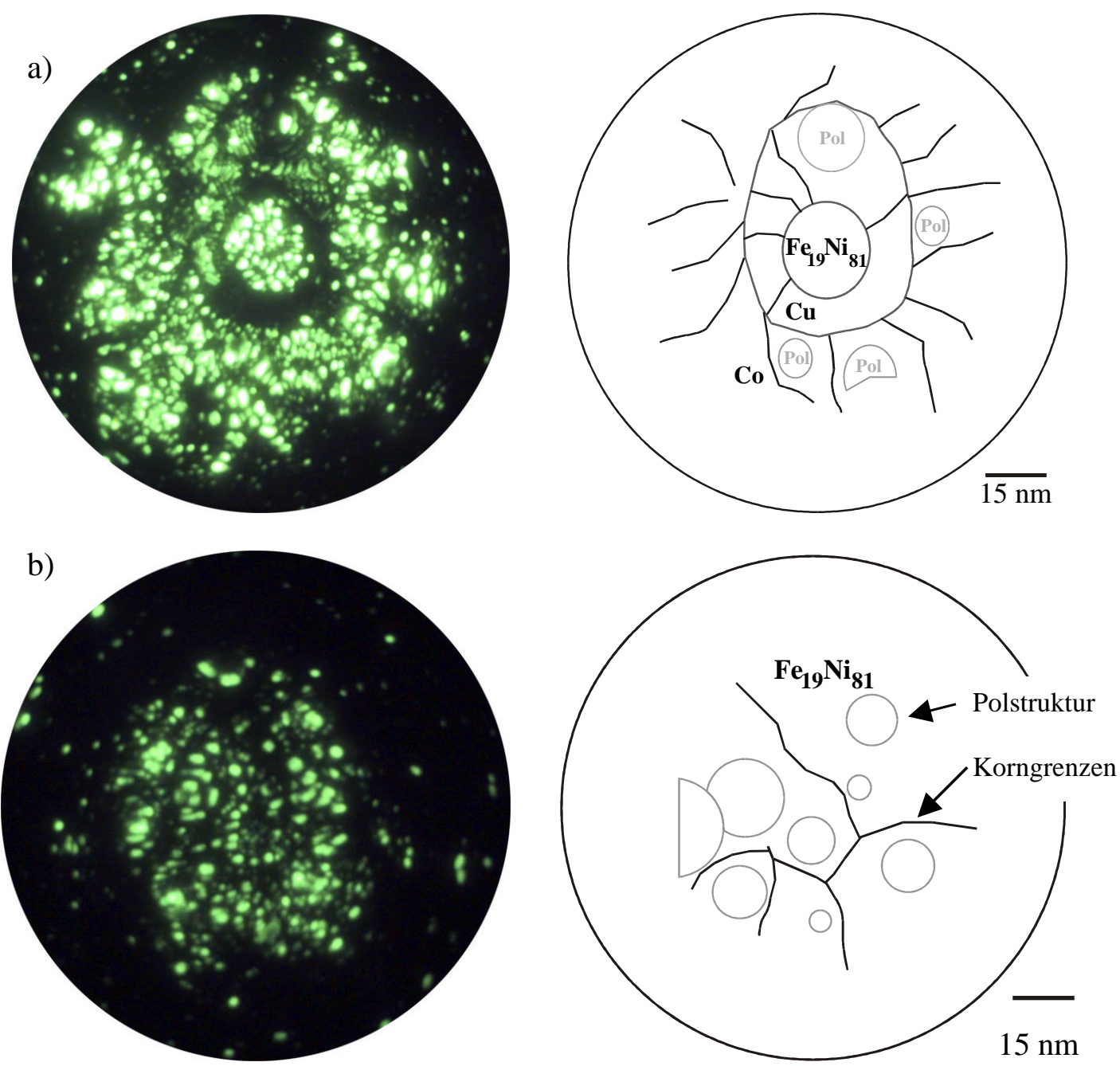

Abbildung 5-3: Feldionenmikroskopische Abbildung eines $\mathrm{Co} / \mathrm{Cu} / \mathrm{Py}-\mathrm{Schich}$ tpaketes. a) Bis zur Py-Schicht feldentwickelte Probe: äußerer Ring-Co; mittlerer Ring-Cu; Py erscheint in der Mitte. b) Voll entwickelte Permalloy-Schicht. Neonpartialdruck $5 \cdot 10^{-5}$ mbar. Rechts sind jeweils die Korngrenzen und Polstrukturen schematisch dargestellt. 
Die laterale Korngröße der Permalloy-Lage verhält sich ähnlich der Co-Schicht im Bereich zwischen 10 und $15 \mathrm{~nm}$. Interessanterweise scheint die $\mathrm{Cu}-\mathrm{Lage}$, trotz der geringeren Schichtdicke, lateral größere Körner auszubilden, die über $15 \mathrm{~nm}$ liegen. Dies liegt vermutlich an der höheren Beweglichkeit des $\mathrm{Cu}$ bedingt durch den sehr viel niedrigeren Schmelzpunkt. Alle durchgeführten TAP-Analysen bestätigen das Bild. In jeder Messung lassen sich innerhalb der $\mathrm{Co}-, \mathrm{Cu}-$ und Py-Schicht sehr leicht ausgedehnte Bereiche mit deutlicher Auflösung der kristallographischen Ebenen ausmachen. Diese werden wie im Kapitel 4 (Ag/Al) beschrieben, zur Kalibrierung der Tiefenskala der Rekonstruktion benutzt.

Zur Analyse der Temperaturstabilität der gesputterten Schichten wurden Wärmebehandlungen in einer speziellen UHV-Kammer durchgeführt, in der der Probenhalter durch zwei $150 \mathrm{~W}$ Halogenstrahler beheizt wird.

Die für die anschließende Analyse in der Tomographischen Atomsonde benutzten Parameter sind in Tabelle 5 aufgeführt.

\begin{tabular}{|c|c|}
\hline \multicolumn{2}{|l|}{ Meßparameter TAP } \\
\hline Pulsverhältnis $\alpha=\mathbf{U}_{\mathbf{P}} /\left(\mathbf{U}_{\mathbf{P}}+\mathbf{U}_{\mathbf{D C}}\right)$ & $17 \%$ \\
\hline Pulsfrequenz & $2000 \mathrm{~Hz}$ \\
\hline Detektionsrate & $\approx 0,008$ Ionen / Puls \\
\hline Detektionseffizienz & 0,5 \\
\hline Temperatur Kühlkopf $^{12}$ & $40 \mathrm{~K}$ \\
\hline Feldverdampfungsfeldstärke Co ${ }^{13}$ & $36 \mathrm{~V} / \mathrm{nm}$ \\
\hline Feldverdampfungsfeldstärke $\mathrm{Cu}^{13}$ & $30 \mathrm{~V} / \mathrm{nm}$ \\
\hline Feldverdampfungsfeldstärke $\mathrm{Fe}^{13}$ & $35 \mathrm{~V} / \mathrm{nm}$ \\
\hline Feldverdampfungsfeldstärke $\mathrm{Ni}^{13}$ & $35 \mathrm{~V} / \mathrm{nm}$ \\
\hline
\end{tabular}

Tabelle 5: Meßparameter der Tomographischen Atomsonde

Um eventuelle Konzentrationsverfälschungen durch bevorzugte Feldverdampfung einer Komponente bei möglicherweise zu hoher Probentemperaturen zu vermeiden, wurden Eichmessungen an einer eingeschmolzenen $\mathrm{Fe}_{20} \mathrm{Ni}_{80}$-Legierung durchgeführt. Die Temperatur der Spitze wurde dabei von $20 \mathrm{~K}$ bis auf $60 \mathrm{~K}$ erhöht, wobei sich keine Abweichung der detektierten Konzentration von den vorher erstellten Mikrosondendaten ergab. Die anschließenden Messungen der Schichtsysteme wurden zur Verbesserung der Stabilität nicht bei tiefsten Temperaturen, sondern bei $40 \mathrm{~K}$ durchgeführt.

\footnotetext{
${ }^{12}$ Durch thermische Verluste beim Ankoppeln des Probenhalters über Kupferlitzen an den Kühlkopf liegt die eigentliche Temperatur an der Spitze etwa 10 - $15 \mathrm{~K}$ höher.

${ }^{13} \mathrm{vgl}$. [MILLER]
} 


\title{
5.3.2 Experimentelle Ergebnisse der Tomographischen Atomsonde
}

Es zeigt sich, daß die Analyse dieses magnetischen Schichtsystems große Anforderungen an die chemische Auflösung der Tomographische Atomsonde stellt.

$\mathrm{Da}$ die Komponenten des $\mathrm{Co} / \mathrm{Cu} / \mathrm{Py}-$ Schichtsystems direkt im Periodensystem benachbart sind und dementsprechend eine sehr ähnliche Masse besitzen, bedarf die Auswertung der detektierten Ereignisse bei der Rekonstruktion besonderer Sorgfalt. Obwohl die in Göttingen betriebene Tomographische Atomsonde eine Massenauflösung von etwa $\mathrm{m} / \Delta \mathrm{m} \approx 150$ (FWHM) besitzt, ist die Trennung von doppelt geladenen Isotopen äußerst schwierig. Wie in Abbildung 5-4 zu sehen, sind die Massenpeaks der $\mathrm{Fe}-, \mathrm{Ni}-$ und $\mathrm{Cu}-$ Isotope leicht zu identifizieren und sauber $\mathrm{zu}$ trennen. Fügt man den $\mathrm{Co}_{59}-\mathrm{Peak}$ hinzu, so kommt es zu einer Überlappung mit dem Ausläufer des $\mathrm{Ni}_{58}-$ Peaks und dem Anfang des $\mathrm{Ni}_{60}-$ Peaks. Insbesondere in dem Fall von Hydridbildung, der mit einer gewissen Häufigkeit bei jeder Messung auftritt, ist eine eindeutige Elementzuordnung nicht mehr möglich. Da das Göttinger TAP nicht über ein Reflektron verfügt, um über Energiekompensation die Massenauflösung zu erhöhen, läßt sich eine chemisch genaue Analyse nur über die Rückrechnung der relativen Häufigkeiten der betroffenen Isotope erreichen. Eine zusätzliche Kontrolle erhält man durch Zerlegung des Schichtsystems in gut auflösbare $\mathrm{Co} / \mathrm{Cu}-$ und $\mathrm{Cu} / \mathrm{Py}-$ Teile. Daher wird zur hochaufgelösten Elementdarstellung der Schichten immer die zweigeteilte Abbildung gewählt.

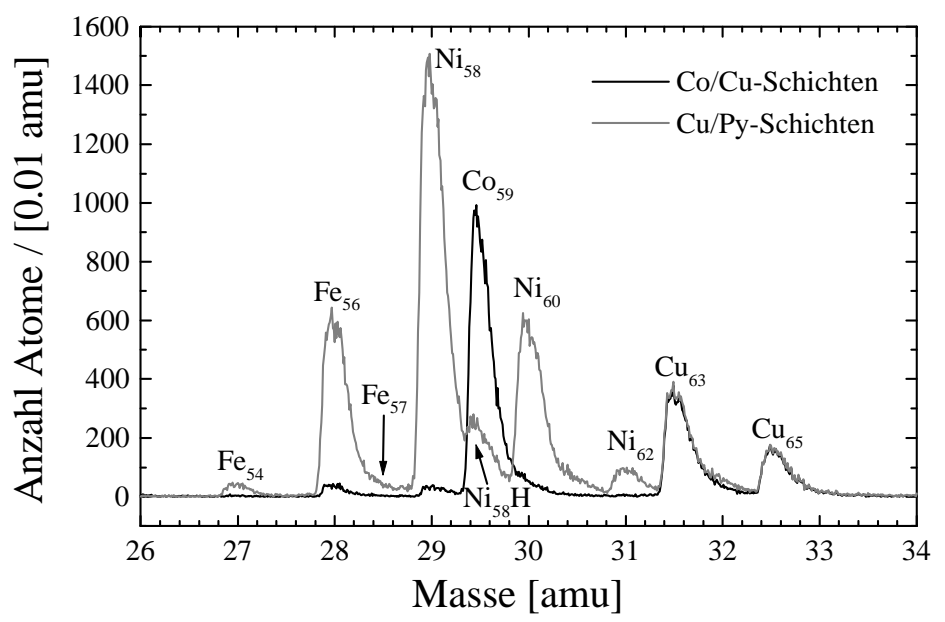

\begin{abstract}
Abbildung 5-4: Massenspektrum eines bei $350^{\circ} \mathrm{C}$ für 30 Minuten wärmebehandelten $\mathrm{Co} / \mathrm{Cu} / \mathrm{Py}-\mathrm{Schichtpaketes.} \mathrm{Von} \mathrm{großer} \mathrm{Bedeutung} \mathrm{für} \mathrm{eine} \mathrm{genaue} \mathrm{Analyse} \mathrm{ist} \mathrm{die} \mathrm{Superposition}$ des Co-Peaks zwischen den Ni-Isotopen.
\end{abstract}

Für erste Untersuchungen zur Analyse thermisch induzierter Veränderungen des Lagensystems wurden deutlich dickere Schichten deponiert, als für technische Anwendungen üblich ist, um auftretende Effekte künstlich zu verstärken.

Abbildung 5-5 a) und b) zeigt die 3D-Rekonstruktion des deponierten $\mathrm{Co}_{10 \mathrm{~nm}} / \mathrm{Cu}_{20 \mathrm{~nm}} / \mathrm{Py}_{25 \mathrm{~nm}}-$ Schichtsystems im Ausgangszustand. Die Trennung in die 
jeweiligen Bilayer ermöglicht eine chemisch genaue Analyse. Die Rekonstruktionen zeigen eine saubere Lagenstruktur.

a)

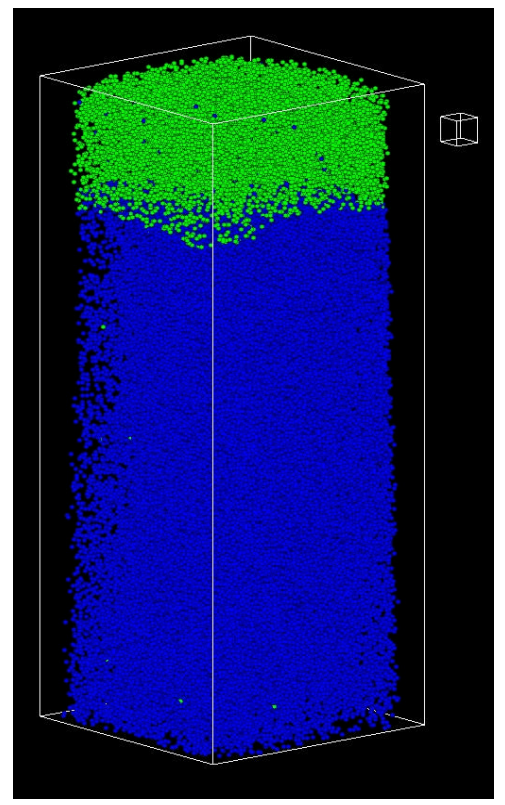

c)

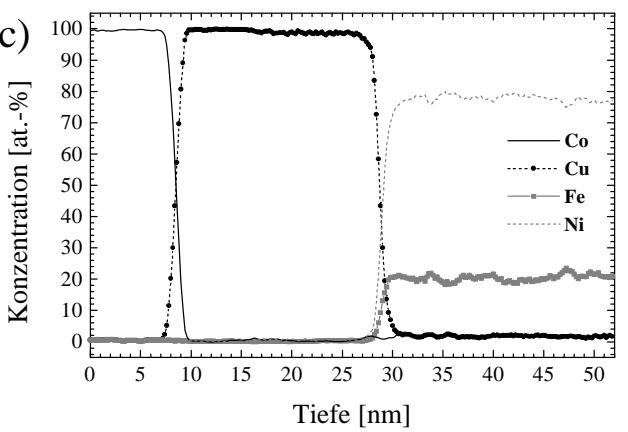

b)

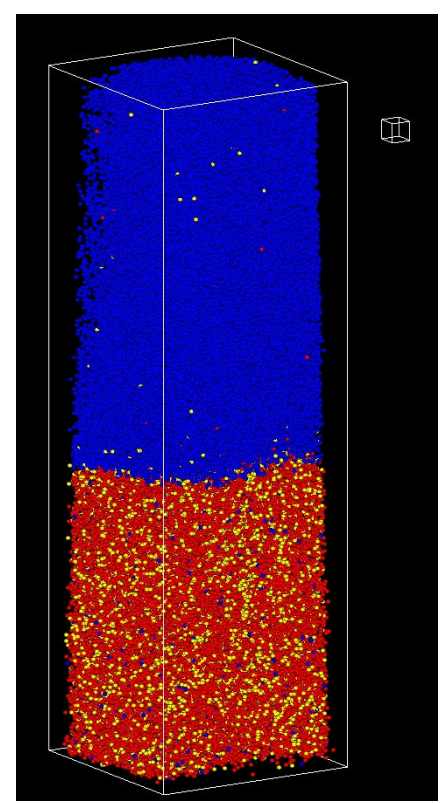

d)

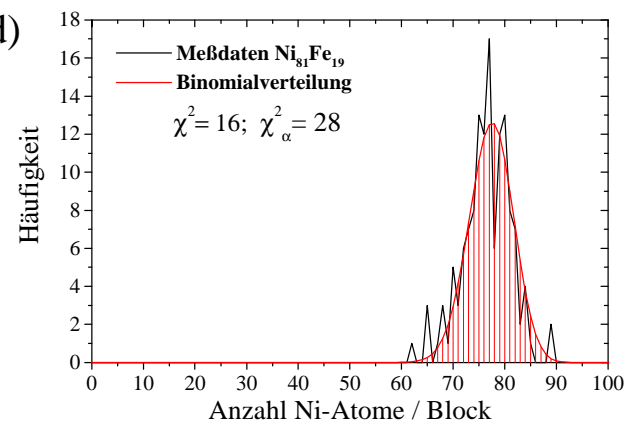

Abbildung 5-5: 3D-Rekonstruktion des $\mathrm{Co} / \mathrm{Cu} / \mathrm{Py}-$ Schichtpaketes im Ausgangszustand. a) $\mathrm{Co} / \mathrm{Cu}$-Lage; b) $\mathrm{Cu} / \mathrm{Py}$-Lage; c) Konzentrationsprofil entlang der Analyse-Achse; d) $\chi^{2}$-Test innerhalb der Permalloy-Schicht.

Mit Hilfe von Isokonzentrationsflächen wurde der zur Konzentrationsanalyse benutzte Zylinder mit höchstmöglicher Genauigkeit senkrecht zu den Schichten justiert, um den Konzentrationsverlauf exakt bestimmen zu können. Trotzdem zeigt der Konzentrationsplot in Abbildung 5-5 c) die Ausbildung von Durchmischungszonen an beiden Grenzflächen von 1 bis 1,5 nm Tiefe. Eine ähnliche Durchmischung wurde ebenfalls in anderen metallischen Schichtsystemen gefunden, wie bereits im diskutierten System Ag/Al [SCHLEIWIES2] oder bei [JESKE1]. Nach durchgeführten Monte-Carlo-Simulationen mit Hilfe des Programms SRIM-2000 ist diese Durchmischung nicht auf die beim Sputterprozeß auftretenden kinetischen Teilchenenergien zurückzuführen. Diese erlauben lediglich eine Eindringtiefe der gesputterten Materialen in die Substratoberfläche bis zu $3 \AA$. Auch das Umschalten zwischen den einzelnen Targets innerhalb von 2 Sekunden führt bei den niedrigen 
Auftragraten höchstens zu einer Durchmischung von $3 \AA$. Es müssen also noch andere Gründe für die ausgedehnte Mischungszone existieren. Wie aus dem Konzentrationsverlauf zu entnehmen ist, wurde auch die Permalloy-Schicht in der richtigen Zusammensetzung, $\mathrm{Fe}_{19} \mathrm{Ni}_{81}$, vom Legierungstarget übertragen. Dies ist für einen Sputterprozeß keineswegs selbstverständlich, aber bei $\mathrm{Fe}-\mathrm{Ni}$ aufgrund ähnlicher Massen und Schmelzpunkte durchaus zu erwarten. Ein $\chi^{2}-$ Test innerhalb der Permalloy-Lage zeigt an, daß die Ni-Atome homogen im Volumen verteilt sind und keinerlei durch die Herstellung verursachte Inhomogenitäten existieren.

Ein Schichtpaket der gleichen Struktur wurde bei $350^{\circ} \mathrm{C}$ für 30 Minuten wärmebehandelt. Diese Temperatur wurde gewählt, da, wie bereits im einleitenden Abschnitt 5.1 vorgestellt wurde, sich die Eigenschaften des Magnetowiderstandes von $\mathrm{Cu} / \mathrm{Co}-$ und $\mathrm{Cu} / \mathrm{Py}-\mathrm{Schich}$ tsystemen in diesem Temperaturbereich anfangen $\mathrm{zu}$ verschlechtern.

In Abbildung 5-6 a) ist die $\mathrm{Co} / \mathrm{Cu}-$ Seite des Trilayers wiedergegeben. Sowohl die $\mathrm{Co}-$ als auch die $\mathrm{Cu}-$ Schicht zeigen durchgehende deutliche (111)-Netzebenen. Zusätzlich ist eine in der Abbildung markierte Korngrenze innerhalb der $\mathrm{Cu}$-Lage zu erkennen, die zwei unterschiedlich orientierte $\mathrm{Cu}-\mathrm{Körner}$ mit einem Kippwinkel von etwa $20^{\circ}$ voneinander trennt. Mit Hilfe der Darstellungssoftware lassen sich die ungewünschten Elemente ausblenden. Teil b) zeigt ebenfalls wieder den $\mathrm{Co} / \mathrm{Cu}-\mathrm{Teil}$ des Schichtpaketes, nur sind in diesem Fall lediglich $\mathrm{Fe}-$ und $\mathrm{Ni}-\mathrm{Atome}$ dargestellt, die offensichtlich aus der untersten hier nicht dargestellten Lage stammen. Eine starke Benetzung der $\mathrm{Cu}$-Korngrenze durch die $\mathrm{Ni}$-Atome ist leicht erkennbar. Erstaunlicherweise zeigt sich zudem eine starke Anreicherung von Fe-Atomen in der $\mathrm{Co} / \mathrm{Cu}-\mathrm{Grenzfläche.} \mathrm{Eine} \mathrm{Analyse} \mathrm{der} \mathrm{detaillierten} \mathrm{Atomverteilung} \mathrm{in} \mathrm{Abbildung}$ 5-6 d) zeigt eine Aufweitung der Grenzflächenschärfe verglichen mit dem Ausgangszustand auf 2,5 nm. Der Fe-Gehalt an der $\mathrm{Co} / \mathrm{Cu}-\mathrm{Grenzfläche} \mathrm{liegt} \mathrm{bei} \mathrm{fast} 14$ at.-\%. Eine leichte $\mathrm{Ni}$-Anreicherung um 1 at.- $\%$ ist dort ebenfalls zu verzeichnen. Vergleicht man den Verlauf der Co-Konzentration und die zueinander versetzte Lage des Fe- und Ni-Peaks, so deutet sich an, daß das Fe innerhalb der Co-Schicht gelöst ist und das Ni hingegen als Anreicherung in der Grenzfläche verbleibt. Da sich das System Co-Fe komplett mischend, $\mathrm{Cu}-\mathrm{Fe}$ hingegen völlig entmischend verhält, wäre der $\mathrm{Fe}$-Antransport über die $\mathrm{Cu}$-Korngrenze und ein anschließendes Mischen mit der Co-Lage durchaus verständlich. Einen direkten Nachweis der Legierungsbildung liefert der tomographische Schnitt durch das Meßvolumen, dargestellt in Abbildung 5-6 c). Zur besseren Orientierung ist der Verlauf der Korngrenze und das $\mathrm{Cu}$-Interface zur $\mathrm{Co}$-Schicht durch eine Isokonzentrationsfläche markiert. Gegenübergestellt sind zwei Schnitte, wobei die farbliche Kodierung des linken von blau: 0 bis rot: 15 at.-\% Fe und die des rechten von blau: 0 bis rot: 100 at.-\% Co reicht. Die Vermutung wird bestätigt, daß durch Eindiffusion des $\mathrm{Fe}$ in die $\mathrm{Co}-$ Schicht eine Legierungszone von etwa 2,5 nm Breite gebildet wurde. 
An der Korngrenze innerhalb der $\mathrm{Cu}-\mathrm{Schicht}$ dreht sich das $\mathrm{Fe} / \mathrm{Ni}$ Verhältnis um. Über eine Breite der Segregationszone von etwa $2 \mathrm{~nm}$ läßt sich ein Ni-Gehalt von fast 12 at.-\% nachweisen.

a)

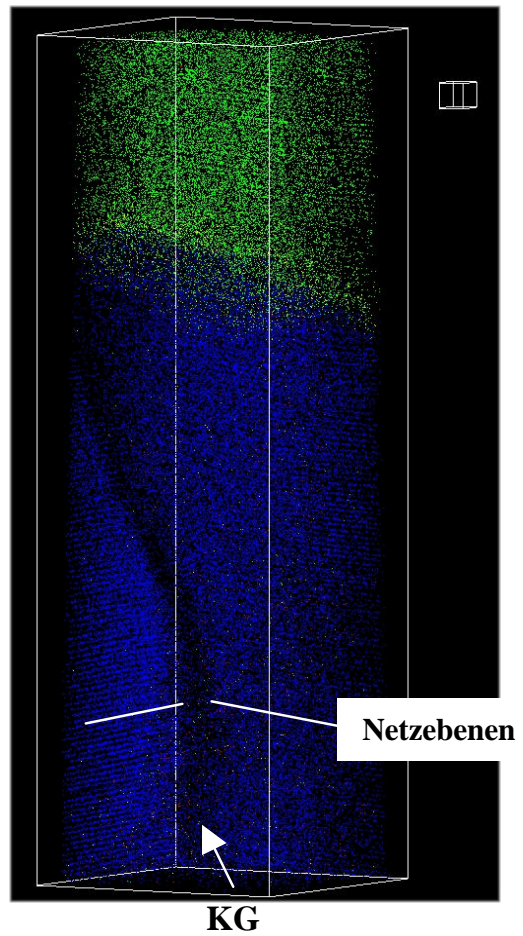

b)

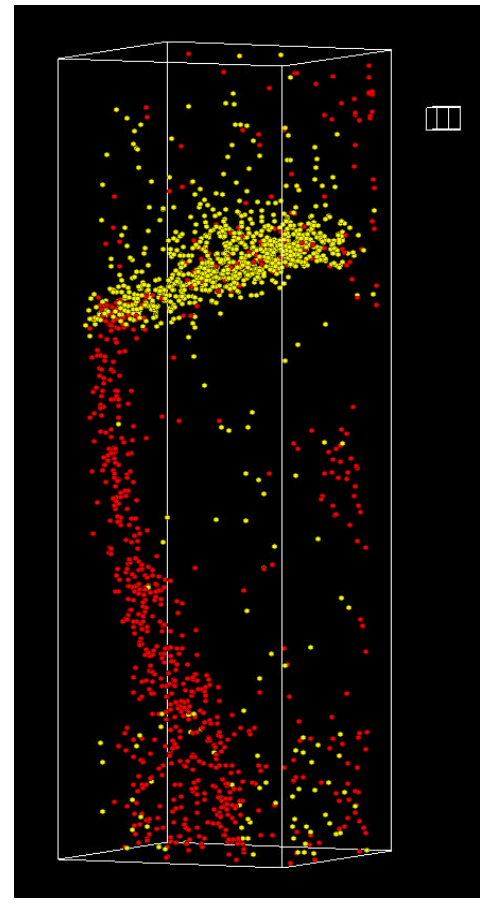

- Co

- $\mathrm{Cu}$

- $\mathrm{Ni}$

$\mathrm{Fe}$

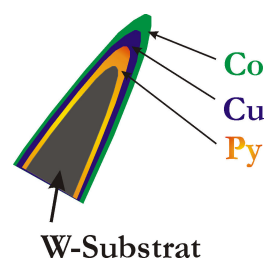

c) 


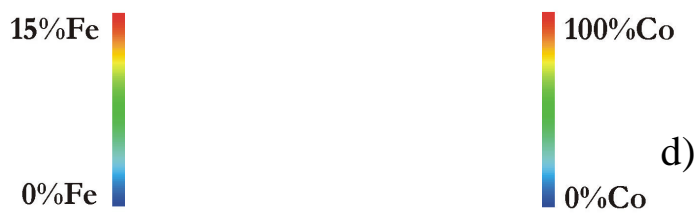

d)
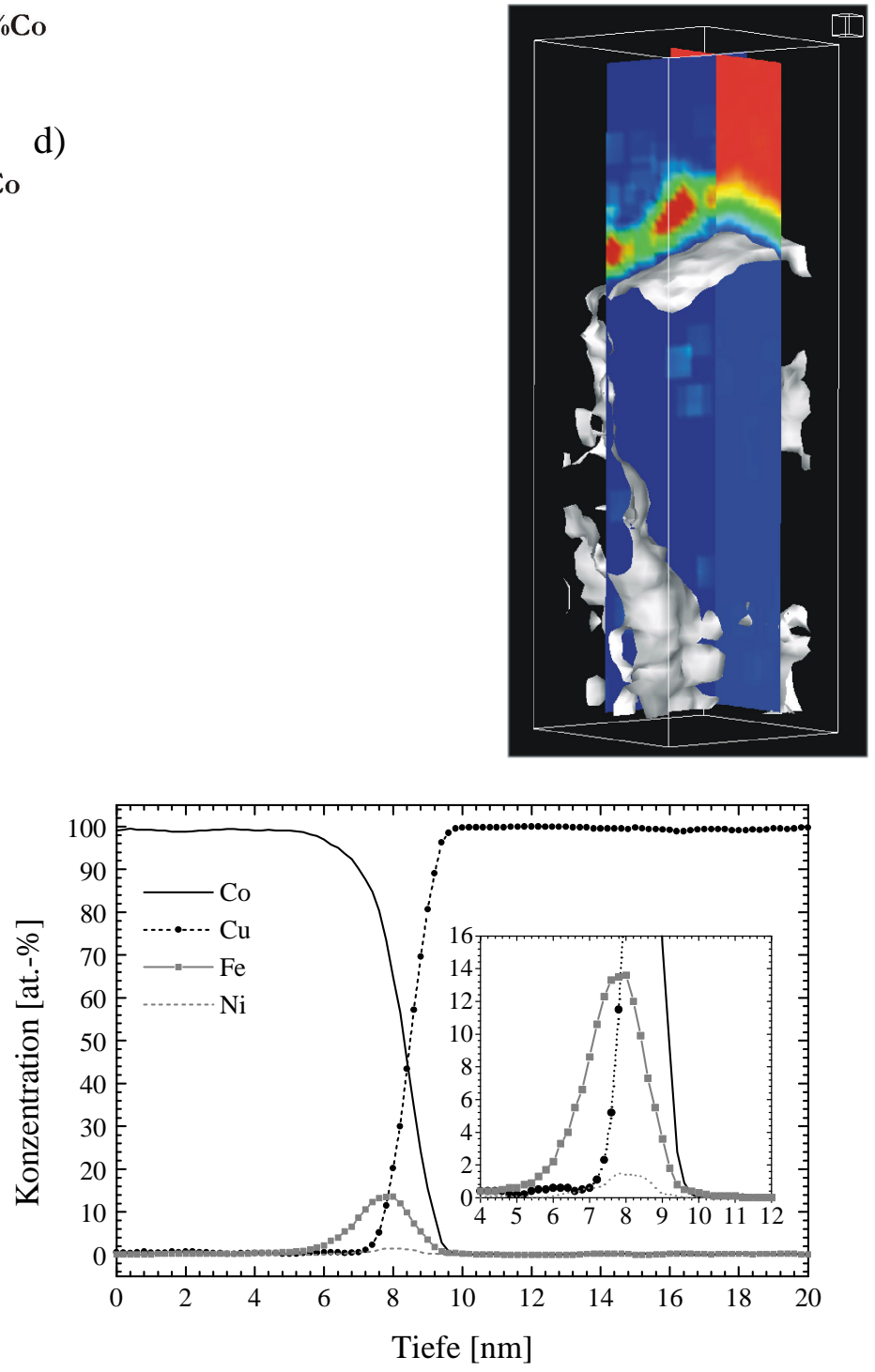

Abbildung 5-6: a) 3D-Rekonstruktion der $\mathrm{Co} / \mathrm{Cu}$-Lagen des bei $350^{\circ} \mathrm{C}$ für 30 Minuten ausgelagerten Trilayers. b) $\mathrm{Fe}-$ und $\mathrm{Ni}$-Atomverteilung innerhalb der $\mathrm{Co} / \mathrm{Cu}-\mathrm{Lage}$. c) Tomographische Schnitte durch die oberen Lagen zur Markierung der CoFe-Legierungsbildung. d) Konzentrationsprofil senkrecht zur $\mathrm{Co} / \mathrm{Cu}-$ Grenzfläche mit Ausschnittsvergrößerung.

Der Fe-Gehalt liegt dagegen mit unter 1 at.-\% schon im statistischen Rauschen. Nicht dargestellt, ist der $\mathrm{Cu} / \mathrm{Py}$-Bilayer, da sich hier keinerlei Veränderung zeigt und nach wie vor eine Unschärfe der Grenzfläche von 1,5 nm vorliegt.

Man könnte kritisieren, daß die gefundenen $\mathrm{Fe}-$ und $\mathrm{Ni}-$ Konzentrationen wegen des zu Anfangs erwähnten Überlapp des $\mathrm{Co}_{59}-$ Massenpeaks mit den beiden Ni-Isotopen nicht signifikant sind oder zumindest mit einem sehr großen Fehler behaftet sein müssen. Dies ist jedoch nicht der Fall. Der im Massenspektrum geplottete Verlauf des $\mathrm{Co} / \mathrm{Cu}$-Bilayers (vgl. Abbildung 5-4) enthält bereits die in Teilbild b) abgebildeten $\mathrm{Fe}-$ und $\mathrm{Ni}$-Atome. Gerade aufgrund ihrer geringen Anteile ist eine 
saubere Trennung der Massenpeaks möglich, was die vorgelegten Daten als exakt bestimmbar untermauert.

Die bisherigen $\mathrm{Cu}$-Schichtdicken lagen außerhalb der bei GMR-Anwendungssystemen interessanten Größen. Um den Einfluß der $\mathrm{Cu}-\mathrm{Schich}$ tdicke auf den Reaktionsverlauf $\mathrm{zu}$ testen, wurde eine dünnere, $4 \mathrm{~nm}$ dicke $\mathrm{Cu}-\mathrm{Schicht}$, die im Anwendungsbereich von Spin-valve Systemen liegt, zwischen die Co- und Py-Lage deponiert. Nach einer Wärmebehandlung bei $250^{\circ} \mathrm{C}$ für 30 Minuten zeigt das aufgenommene Konzentrationsprofil in Abbildung 5-7 ganz ähnliche Eigenschaften wie das vorher für $350^{\circ} \mathrm{C}$ diskutierte. Gleichfalls wird eine $\mathrm{Fe}$-Eindiffusion in die $\mathrm{Co}-$ Lage am $\mathrm{Co} / \mathrm{Cu}$-Interface beobachtet.

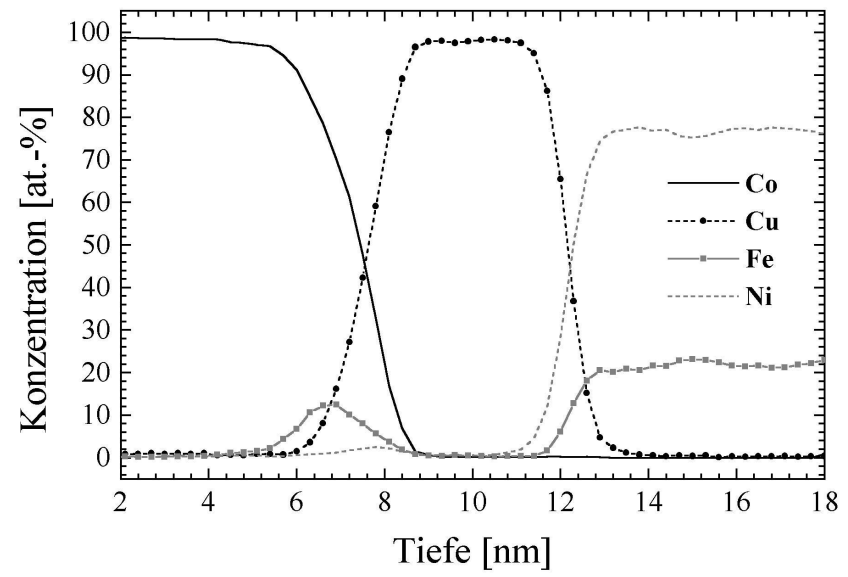

Abbildung 5-7: Konzentrationsprofil des bei $250^{\circ} \mathrm{C}$ für 30 Minuten ausgelagerten $\mathrm{Co} / \mathrm{Cu}_{4}$ nm $/ \mathrm{Py}-$ Schichtsystems.

Der Fe-Gehalt liegt in dieser Analyse bei 12 at.-\% ausgedehnt über eine Tiefe von etwa $2 \mathrm{~nm}$. Im Gegensatz zur vorher angesprochenen Probe wurden in dem analysierten Volumen keine strukturellen Hinweise auf eine Korngrenze gefunden, noch eine Markierung dieses Gitterfehlers durch segregierende $\mathrm{Fe}-$ oder $\mathrm{Ni}$-Atome festgestellt. Allerdings zeigte sich eine durchgehende homogene $\mathrm{Ni}$-Anreicherung der $\mathrm{Cu}-\mathrm{Schicht}$ um bis zu 2,5 at.-\%.

In den bisherigen Analysen scheint ein grundlegender Mechanismus der Schichtreaktion hervorzutreten. Die vorgestellten, und weitere durchgeführte Kontrollmessungen zeigen alle das Anreicherungsverhalten von $\mathrm{Fe}$ in der Co-Grenzlage. Diese Eindiffusion gewinnt an Bedeutung, wenn man bedenkt, daß in koppelnden Systemen die Schichtdicken weiter reduziert sind. Je nach Kombination der beteiligten Komponenten liegen bei Anwendungsschichten die $\mathrm{Cu}-\mathrm{Zwischenschichtdicken} \mathrm{im} \mathrm{2.} \mathrm{Kopplungsmaximum} \mathrm{bei} \mathrm{1,7} \mathrm{bis} 2 \mathrm{~nm}$. Auch die anderen ferromagnetischen Lagen sind im koppelnden Fall üblicherweise unter $4 \mathrm{~nm}$ 
dick. Aufgrund der limitierten Verfügbarkeit der Komponenten hätte dann die bevorzugte Diffusion einer Komponente extreme Folgen.

Aus diesem Grund wurde zur Kontrolle ein Schichtpaket, bestehend aus den entsprechend geringen Lagendicken, hergestellt. Die 3D-Rekonstruktion des bei $350^{\circ} \mathrm{C}$ für 30 Minuten ausgelagerten $\mathrm{Co} / \mathrm{Cu}_{1,7 \mathrm{~nm}} / \mathrm{Py}_{3 \mathrm{~nm}} / \mathrm{Cu}_{1,7 \mathrm{~nm}} / \mathrm{Co}-\mathrm{Schich}$ tsystems ist in Abbildung 5-8 a) dargestellt. Wie in den Messungen zuvor, zeigt auch diese Probe eine $\mathrm{Fe}-$ Anreicherung an den zwei vorhandenen $\mathrm{Co} / \mathrm{Cu}-\mathrm{Grenzflächen.}$ Aufgrund der verringerten $\mathrm{Cu}$-Schichtdicke ist der $\mathrm{Fe}-$ Gehalt in der Co-Lage auf nahezu 18 at.-\% angestiegen. Die Legierungszone hat eine Ausdehnung von etwa 1,5 nm. Nicht vergessen darf man dabei, daß die $3 \mathrm{~nm}$ dicke Permalloy-Schicht die Zusammensetzung $\mathrm{Fe}_{19} \mathrm{Ni}_{81}$ besitzt. Tatsächlich zeigt eine derart starke Fe-Diffusion Auswirkungen auf die Zusammensetzung der Permalloy-Schicht. Innerhalb der Lage ist ein Absinken des Fe-Gehaltes im Extremfall auf bis zu 15 at.-\% zu beobachten. Die Legierung hat sich in ihrer Zusammensetzung also drastisch verändert, was natürlich auch deren magnetische Eigenschaften beeinflußt. In der Regel sind die magnetischen Sensoren in ihrer Schichtdicke auf maximale Kopplungsstärke optimiert, die natürlich von den beteiligten Komponenten abhängt. Die Veränderung einer Komponente zieht demnach auch die Änderung der Kopplungsstärke nach sich. Zusätzlich ist die $\mathrm{Cu}-$ Schicht durch die Diffusion derart von anderen Elementen durchsetzt, daß im Konzentrationsprofil nur noch 70 at.-\% $\mathrm{Cu}$ erreicht werden. Anscheinend liegt auch an der $\mathrm{Py} / \mathrm{Cu}-\mathrm{Grenzfläche} \mathrm{Ni}-\mathrm{Zulegierung}$ in der $\mathrm{Cu}-$ Lage vor, die den leicht geknickten Verlauf der Ni-Konzentration erklärt. 


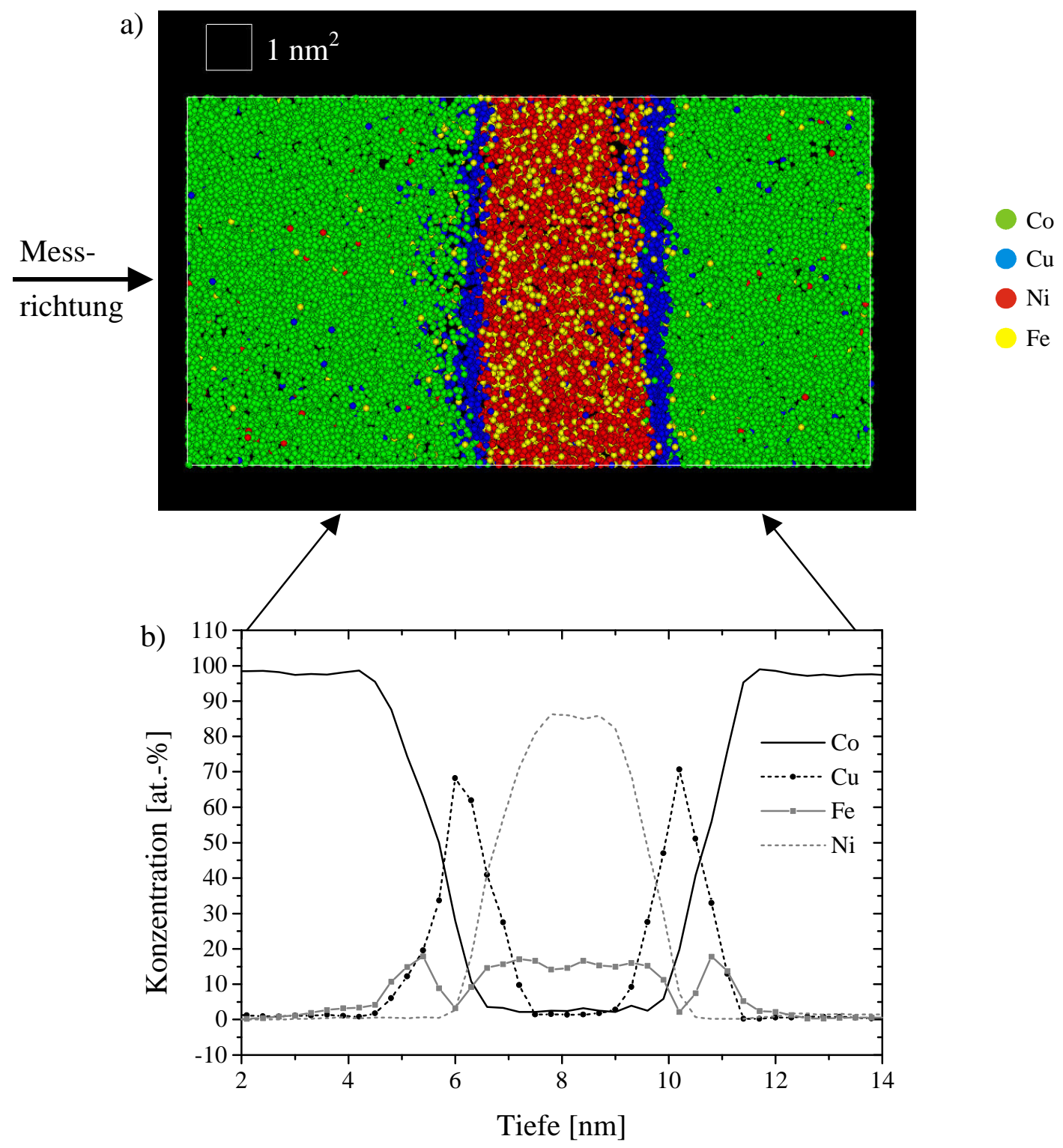

Abbildung 5-8: a) 3D-Rekonstruktion der bei $350^{\circ} \mathrm{C}$ für 30 Minuten ausgelagerten $\mathrm{Co} / \mathrm{Cu}_{1,7 \mathrm{~mm}} / \mathbf{P y}_{3 \mathrm{~nm}} / \mathrm{Cu}_{1,7 \mathrm{~nm}} / \mathrm{Co}-$ Probe. b) Konzentrationsprofil entlang der Meßrichtung.

An dieser Stelle kann natürlich kritisiert werden, daß die dargestellten Messungen nicht mit realen Systemen planarer Schichtstruktur korrelierbar sind. Auch eine Diskussion der in der Literatur vorliegenden Messungen des magnetischen Widerstandes auf der Grundlage der gewonnenen strukturellen Daten wäre dann nicht überzeugend. Um einer solchen Kritik $\mathrm{zu}$ begegnen, wurden planare $\left[\mathrm{Co}_{10 \mathrm{~nm}} / \mathrm{Cu}_{10 \mathrm{~nm}} / \mathrm{Py}_{3,5 \mathrm{~nm}} / \mathrm{Cu}_{10} \mathrm{~nm}_{20}\right.$-Teststrukturen, die in der Bielefelder Gruppe von Prof. Reiss hergestellt wurden, mit der beschriebenen Methode der ElektronenstrahlLithographie strukturiert und zur analysierbaren TAP-Spitze präpariert. 


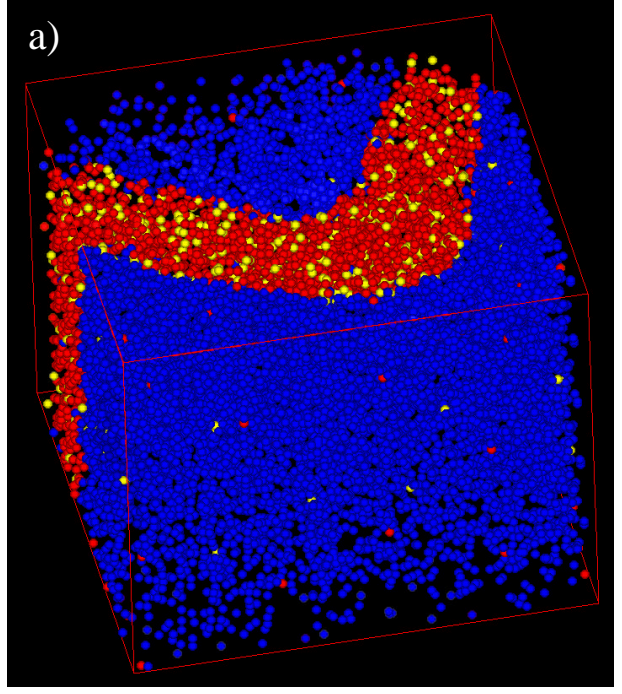

b)

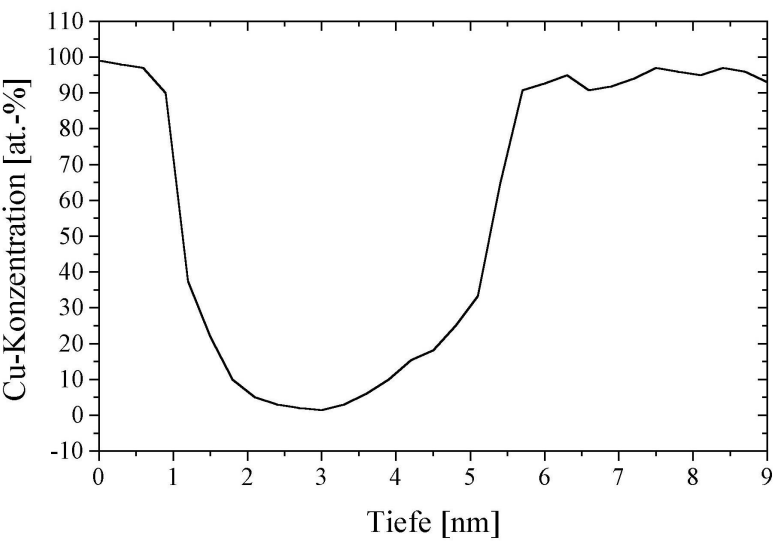

$\mathrm{Co}$

Meßrichtung
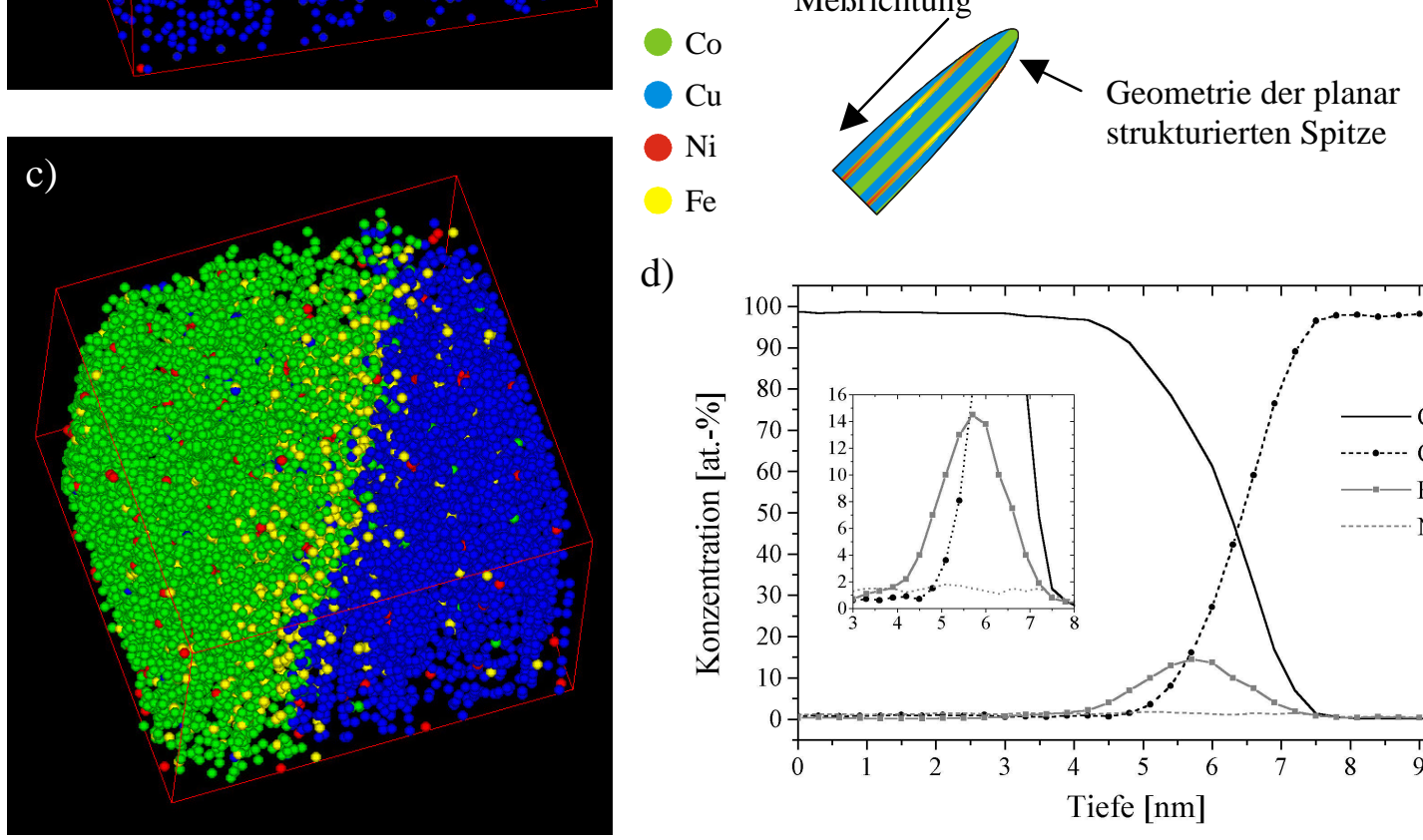

d)

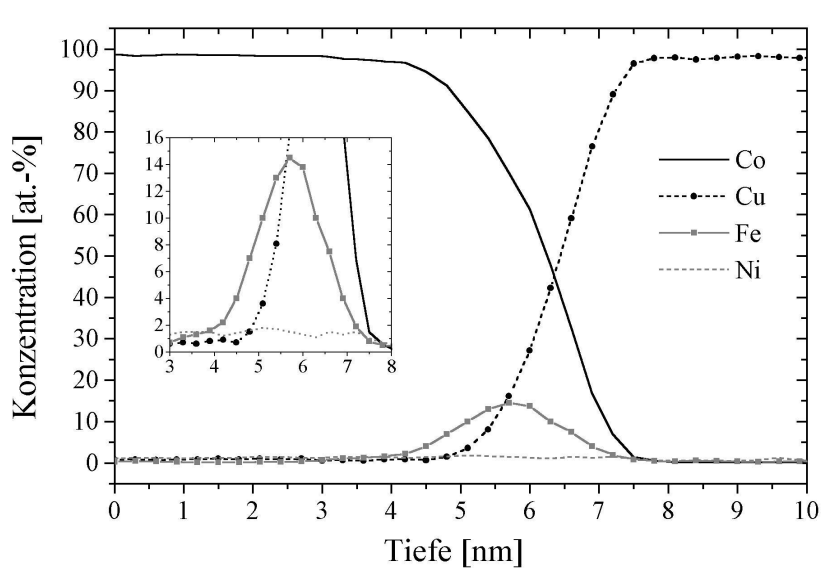

Abbildung 5-9: a) 3D-Rekonstruktion der lithographisch strukturierten $[\mathrm{Co} / \mathrm{Cu} / \mathbf{P y} / \mathrm{Cu}]-$ planaren Probe im Ausgangszustand. b) Konzentrationsprofil senkrecht zur Schichtfolge. c) Rekonstruktion der bei $\mathbf{3 5 0}^{\circ} \mathrm{C}$ für 30 Minuten wärmebehandelten planaren Probe. d) Konzentrationsprofil senkrecht zur Schichtfolge.

Im Gegensatz zu den vorher diskutierten, auf W-Trägerspitzen aufgesputterten Schichten, verläuft die Analyse nicht senkrecht durch die Lagen hindurch, sondern parallel zu ihren Grenzflächen. In Abbildung 5-9 a) ist die Rekonstruktion des Ausgangszustandes dargestellt. Aufgrund der relativ großen Schichtdicken und des limitierten Analysevolumens der Tomographischen Atomsonde, das in der Regel bei $10 \cdot 10 \cdot 100 \mathrm{~nm}^{3}$ liegt, ist eine Erfassung der gesamten Schichtperiodizität nicht möglich. Deshalb ist es nicht verwunderlich, daß in dieser Messung nur der $\mathrm{Cu} / \mathrm{Py} / \mathrm{Cu}-$ Ausschnitt detektiert wurde. Wie die Rekonstruktion zeigt, sind die Lagen in diesem Bereich wellenförmig moduliert. Aus der Literatur [SCHLEIWIEs1, TROCHE] ist bekannt, daß bei der Deposition von Multilagenpaketen mit zunehmender Lagenzahl und Dicke, Störungen im planaren Wachstum auftreten können, die schließlich in 
starken sinusförmigen Modulationen der zuletzt aufgebrachten Lagen resultieren. Nimmt man an, daß im Fall der Untersuchung des Ausgangszustandes ein Probengebiet nahe der Oberfläche analysiert wurde, ist die gefundene starke Welligkeit verständlich. Die hohe Auflösung der Methode ermöglicht auch an diesen gekrümmten Schichtfolgen atomar genaue chemische Analysen. In Abbildung 5-9 b) ist eine Konzentrationsbestimmung senkrecht $\mathrm{zu}$ den $\mathrm{Cu} / \mathrm{Py} / \mathrm{Cu}-\mathrm{Grenzflächen}$ wiedergegeben. Sie zeigt einen chemischen Übergangsbereich von etwa $0,8 \mathrm{~nm}$ an den Grenzflächen.

Nach der Wärmebehandlung einer lithographisch strukturierten Spitze bei $350^{\circ} \mathrm{C}$ für 30 Minuten, also unter ähnlichen Bedingungen wie bei den vorher diskutierten Proben, zeigen sich entsprechende Charakteristika. Das analysierte Volumen zeigt die $\mathrm{Co} / \mathrm{Cu}-$ Grenzfläche. Eine Welligkeit, wie in der Probe des Ausgangszustandes, ist nicht zu beobachten. Wie augenscheinlich der Rekonstruktion zu entnehmen ist, gibt es eine deutliche $\mathrm{Fe}-$ Anreicherung in der $\mathrm{Co}-$ Lage an der $\mathrm{Co} / \mathrm{Cu}-\mathrm{Grenzfläche.}$ Die Bestimmung des Konzentrationsverlaufes, dargestellt in Teilbild d), bestätigt diesen Eindruck. Nahezu 15 at.-\% Fe sind in der Co-Grenzschicht gelöst. Ein vernachlässigbarer $\mathrm{Ni}-$ Gehalt von unter 2 at.-\% ist ebenfalls festzustellen.

Die präparierten planaren Proben zeigen also in keiner Hinsicht ein anderes Reaktionsbild, als die auf W-Trägerspitzen aufgebrachten Schichtsysteme. Deren Ergebnisse können also sehr wohl zur Korrelation mit anderen, an planaren Systemen gewonnenen Meßdaten herangezogen werden.

\subsection{Diskussion der Ergebnisse}

Die hier vorgestellten mikrostrukturellen Analysen von bereits in der Anwendung befindlichen GMR-Schichtsystemen ermöglichen erstmals einen Einblick in die atomare Umverteilung der beteiligten Komponenten bei thermischer Anregung. Nur durch die detaillierte Kenntnis der atomaren Struktur ist eine eindeutige Interpretation der in der Literatur vorhandenen Messungen des Magnetowiderstandes in Abhängigkeit von der Temperatur sinnvoll möglich.

Am auffälligsten ist die $\mathrm{Fe}-$ Diffusion durch die $\mathrm{Cu}$-Lage an die $\mathrm{Co} / \mathrm{Cu}-\mathrm{Grenzfläche}$ und anschließende Legierungsbildung innerhalb der Co-Schicht, die zu einer signifikanten Fe-Segregation führt. Auf diese Weise erfolgt schon bei niedrigen Temperaturen das diskutierte Grenzflächendusting, also die Segregation einer geringen Menge einer fremden Komponente an die Grenzfläche. Das ist möglicherweise von großem Vorteil, da man ein Grenzflächendusting durch eine einfache Wärmebehandlung erzielen könnte, ohne die Wachstumsbedingungen zu stören. Nach den in der Einleitung erwähnten, von MERTIG und ZAHN durchgeführten Rechnungen sollte das Einfügen einer dünnen Fe-Lage in die grenzschichtnahe $\mathrm{Co}-$ Schicht eine Erhöhung der GMR-Effektamplitude zur Folge haben. In Abbildung 5-10 sind die in der Bielefelder Gruppe aufgenommenen Magnetowiderstandskurven eines $\mathrm{Co} / \mathrm{Cu} / \mathrm{Py}-\mathrm{Schich}$ tpaketes dargestellt. Die Effektamplitude bei Raumtemperatur erreicht im Ausgangszustand etwa 5,5\%. Nach 
einer Wärmebehandlung bei $120^{\circ} \mathrm{C}$ für $10 \mathrm{~h}$ zeigt sich bereits eine deutliche Abnahme des Effektes, der mit weiteren Auslagerungsschritten kontinuierlich sinkt.

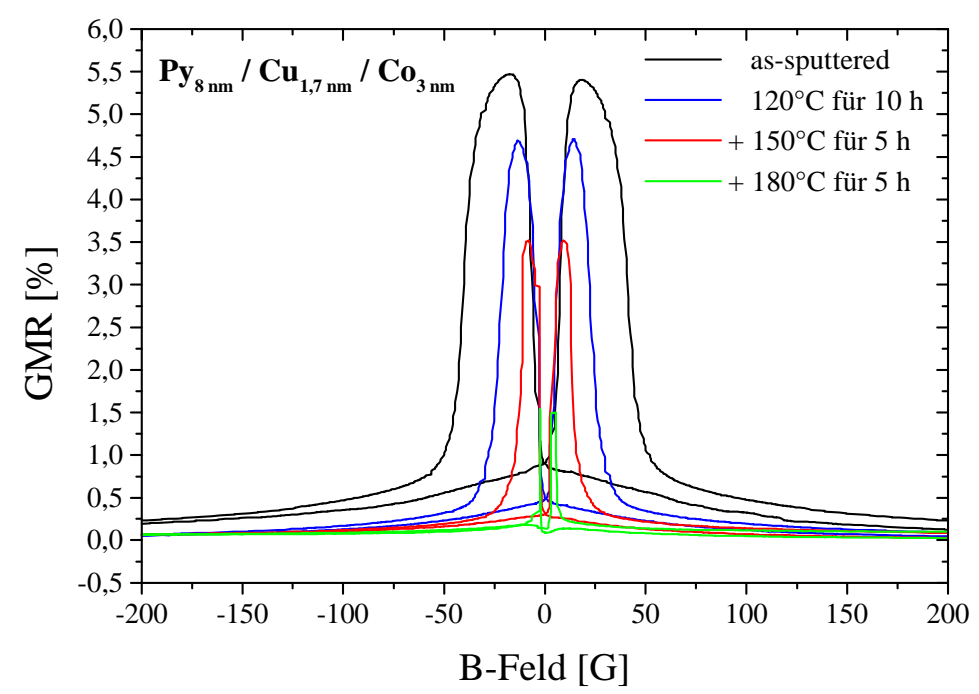

Abbildung 5-10: GMR-Kennkurven eines $\mathrm{Co} / \mathrm{Cu} / \mathrm{Py}-$ Schichtpaketes im Ausgangszustand und
nach verschiedenen additiven Wärmebehandlungen.

Zwar sind die benutzten Temperaturen niedriger als bei den Wärmebehandlungen der TAP-Spitzen, aber aufgrund der langen Zeiten sind die magnetischen Messungen und die Analyse der Atomverteilungen eventuell vergleichbar.

Die Ursache für das starke Abfallen der GMR-Amplitude zu langen Zeiten und hohen Temperaturen, wie sie auch von anderen Autoren bestimmt wurde [HwANG, Heitmann2], läßt sich nun eindeutig zuordnen. Anhand der Messung des $\mathrm{Co} / \mathrm{Cu} / \mathrm{Py} / \mathrm{Cu} / \mathrm{Co}-\mathrm{Schich}$ tpaketes konnte gezeigt werden, daß verursacht durch die starke $\mathrm{Fe}-$ Diffusion an die $\mathrm{Co} / \mathrm{Cu}-\mathrm{Grenzfläche,} \mathrm{der} \mathrm{Fe}-$ Gehalt in der Py-Schicht drastisch abnimmt. Die Veränderung der Zusammensetzung der Legierung bewirkt konsequent eine Änderung der Bandstruktur und dementsprechend auch der Spinasymmetrie. Wie im einleitenden Abschnitt 5.1 beschrieben wurde, verändern sich mit der Bandstruktur auch die Kopplungslängen. Das heißt, mit zunehmender Abnahme des $\mathrm{Fe}-$ Gehaltes gerät das Schichtsystem immer stärker aus der Oszillationsperiode, was einen deutlichen Rückgang der GMR-Amplitude zur Folge hat.

Leider stehen zur Zeit keine Messungen des Magnetowiderstandes zur Verfügung, die zeigen könnten, ob bei kleinen Zeiten zunächst eine Erhöhung des GMR-Effektes durch Grenzflächendusting zu verzeichnen ist.

Die beobachtete Asymmetrie der bei der Rekonstruktion gefundenen Verteilung von $\mathrm{Fe}-$ und $\mathrm{Ni}$-Atomen, spiegelt die unterschiedliche Thermodynamik der beteiligten Systeme wieder. Ni und $\mathrm{Cu}$ bilden ein vollständig mischendes System, während die Lösung von $\mathrm{Fe}$ in $\mathrm{Cu}$ sehr gering ist. Deswegen ist es nicht verwunderlich, daß sich während der Wärmebehandlung auch bei niedrigen Temperaturen ein gewisser 
Anteil $\mathrm{Ni}$ im $\mathrm{Cu}$ löst und in den $\mathrm{Cu}-$ Korngrenzen segregiert, wohingegen $\mathrm{Fe}-\mathrm{Atome}$ nur an der $\mathrm{Co} / \mathrm{Cu}-\mathrm{Grenzfläche} \mathrm{zu} \mathrm{detektieren} \mathrm{sind.} \mathrm{Die} \mathrm{Lösung} \mathrm{des} \mathrm{Ni}$ in den $\mathrm{Cu}$-Schichten würde auch eine Erhöhung des Restwiderstandes erklären, wie es von LOYEN bei der Untersuchung von $\mathrm{Cu} / \mathrm{Py}-\mathrm{Schich}$ tsystemen beobachtet wurde [LOYEN].

Bei höheren Temperaturen zeigt sich ein signifikanter $\mathrm{Ni}-\mathrm{Gehalt}$ in den $\mathrm{Cu}-$ Korngrenzen. Dieser läßt sich als Vorläufer der Bildung einer ferromagnetischen Brücke zwischen den Lagen interpretieren, die schließlich zum Zerstören des Magnetowiderstandes führt. Ein über die Korngrenze ablaufender Mechanismus wird seit längerem in der Literatur für den Zusammenbruch des Magnetowiderstandes verantwortlich gemacht. Im Gegensatz $\mathrm{zu}$ den von RÄTZKE an $\mathrm{Co} / \mathrm{Cu}-\mathrm{Schich}$ tsystemen durchgeführten TEM-Untersuchungen, die über einen Diffusionsmechanismus der $\mathrm{Cu}-$ Atome aus der Zwischenschicht in die Korngrenzen der Co-Schicht berichten, was zur Entkoppelung der einzelnen Körner der Lage und damit zum Zusammenbruch des GMR-Effektes führt, zeigt die Nanoanalyse in dieser Arbeit einen anderen Mechanismus auf. Nicht die $\mathrm{Cu}$-Atome der Zwischenschicht selber führen aufgrund ihrer Mobilität zum Verschwinden des Magnetowiderstandes, sondern die Ausbildung eines Segregationspfades der $\mathrm{Fe}-$ und $\mathrm{Ni}$-Atome durch die $\mathrm{Cu}$-Korngrenze. Damit verbunden ist eine Fe-Verarmung der Py-Lage und die Formierung einer ferromagnetischen Brücke durch den starken $\mathrm{Ni}-$ Gehalt der Korngrenze. Beides führt zur Abnahme und schließlich zum Zusammenbruch des GMR-Effektes.

Bestätigt wird dieser Mechanismus durch die experimentell nachgewiesene Abhängigkeit der Temperaturstabilität von der $\mathrm{Cu}-\mathrm{Z}$ wischenschichtdicke.

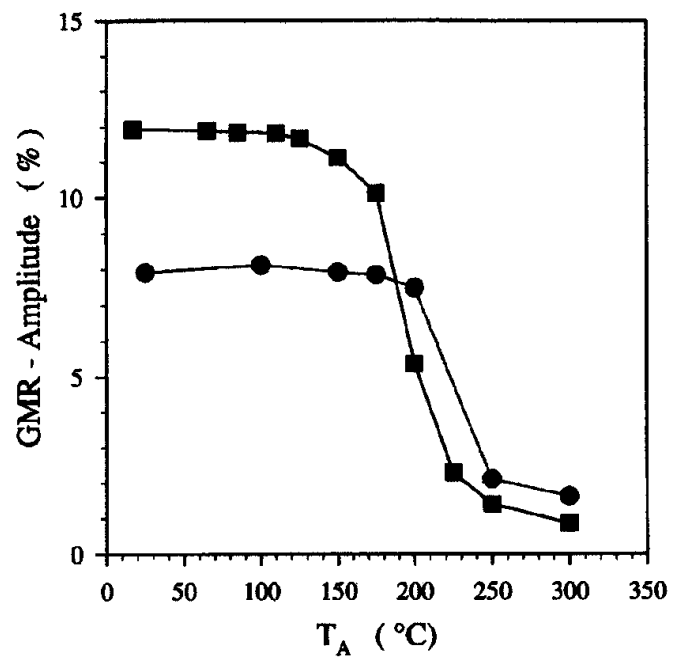

Abbildung 5-11: Entwicklung des GMR-Effektes bei Wärmebehandlung für unterschiedliche $\mathrm{Cu}$-Zwischenschichtdicken eines $\mathrm{Cu} / \mathbf{P y}-$ Schichtsystems: • - $\left(\mathbf{C u}_{\mathbf{1 , 8} \mathbf{~ n m}} / \mathbf{P y}_{\mathbf{1 , 6} \mathbf{~ n m}}\right) /$ - - $\left(\mathrm{Cu}_{0,9} \mathbf{n m} / \mathbf{P y}_{1,6} \mathrm{~nm}\right)$ [Heitmann2].

In Abbildung 5-11 ist die Entwicklung der Magnetowiderstände bei unterschiedlichen $\mathrm{Cu}-Z$ wischenschichtdicken im System $\mathrm{Cu} / \mathrm{Py}$ in Abhängigkeit von 
der Temperatur aufgetragen. Aufgrund stärkerer Austauschwechselwirkung zeigt das Schichtpaket mit der $\mathrm{Cu}-\mathrm{Zwischenschichtdicke} \mathrm{im} \mathrm{ersten} \mathrm{Antiferromagnetischen-}$ Kopplungsmaximum $A F K M(\mathrm{~d}=0,9 \mathrm{~nm}, \quad$-Meßwerte $)$ einen sehr viel höheren GMR-Effekt als die Probe mit $\mathrm{Cu}-$ Schichtdicke von 1,8 nm (2.tes AFKM, --Meßwerte). Bei der Temperaturstabilität offenbart sich jedoch ein umgekehrtes Verhalten. Bereits ab einer Temperatur von $110^{\circ} \mathrm{C}$ fängt der Magnetowiderstand der Probe im ersten AFKM an $\mathrm{zu}$ sinken und trifft den immer noch konstanten GMR-Wert der andern Probe bei $180^{\circ} \mathrm{C}$. Erst bei einer Auslagerung bei Temperaturen über $200^{\circ} \mathrm{C}$ beginnt auch die GMR-Amplitude der Probe im zweiten AFKM abzunehmen.

Diese klare Schichtdickenabhängigkeit der thermischen Reaktion spiegelt sich ebenfalls in den gemessenen Fe-Konzentrationen wieder, die in der Co-Lage an der $\mathrm{Co} / \mathrm{Cu}-\mathrm{Grenzfläche} \mathrm{gelöst} \mathrm{sind} \mathrm{(vgl.} \mathrm{Tabelle} \mathrm{6).} \mathrm{Die} \mathrm{Angabe} \mathrm{der} \mathrm{Fe-Konzentration}$ alleine gibt jedoch keine Auskunft über die absolute Anzahl segregierter Fe-Atome. Ein geeigneterer Vergleichswert ist der Korngrenzen-Exzess $\Gamma_{0}$ (vgl. Abschnitt 3.1.3), der über die absolut vorhandene Atom-Anzahl pro Flächeneinheit Auskunft gibt.

Bestätigend ist mit abnehmender Schichtdicke eine deutliche Zunahme der in der Co-Lage gelösten Fe-Atome festzustellen. Das Ansteigen des Exzess mit abnehmender $\mathrm{Cu}$-Dicke weist klar auf einen kinetischen Effekt und nicht auf eine Gleichgewichtssegregation hin, die das Einstellen eines konstanten Exzess erwarten lassen würde. Dies ist durchaus verständlich, da der größte Anteil des Fe sich nicht in der Korngrenze befindet, sondern aufgrund der vollständigen Mischbarkeit des Systems Co-Fe in der Co-Lage gelöst ist.

\begin{tabular}{|c|c|c|c|}
\hline $3^{\circ} 0^{\circ} \mathrm{C} / 30$ Min & 20 nm Cu-Schicht & $10 \mathrm{~nm} \mathrm{Cu}-$ Schicht & 1,7 nm Cu-Schicht \\
\hline $\begin{array}{l}\text { max. Fe-Gehalt in } \\
\text { Co-Lage [at.-\%] }\end{array}$ & 13,8 & 15 & 18 \\
\hline $\begin{array}{l}\text { Korngrenzen-Exzess } \\
\Gamma_{0}\left[\text { Atome } / \mathrm{cm}^{2}\right]\end{array}$ & $19,9 \cdot 10^{14}$ & $22,3 \cdot 10^{14}$ & $24,7 \cdot 10^{14}$ \\
\hline
\end{tabular}

Tabelle 6: Aus TAP-Analysen bestimmter Fe-Gehalt in der Co-Lage an der $\mathrm{Co} / \mathrm{Cu}-\mathrm{Grenzfläche} \mathrm{und} \mathrm{berechneter} \mathrm{Korngrenzen-Exzess.}$

\subsection{Zusammenfassung der Analyse von $\mathrm{Co} / \mathrm{Cu} / \mathrm{Py}-\mathrm{Schichten}$}

Mit Hilfe der höchstauflösenden Methode der Atomsondentomographie konnte am $\mathrm{Co} / \mathrm{Cu} / \mathrm{Py}-\mathrm{Schich}$ tsystem eindrucksvoll ein Segregationsmechanismus von $\mathrm{Fe}-$ und $\mathrm{Ni}$-Atomen durch die Korngrenze der $\mathrm{Cu}$-Zwischenschicht aufgedeckt werden, der im fortgeschrittenen Stadium zur Abnahme und schließlich zum Zusammenbruch des Magnetowiderstandes führt. Es liegt nahe die experimentellen Ergebnisse derart zu deuten, daß die Veränderung der Bandstruktur durch eine starke Fe-Verarmung der Py-Schicht, verursacht durch die Diffusion und Legierungsbildung innerhalb der 
Co-Lage an der $\mathrm{Co} / \mathrm{Cu}-$ Grenzfläche, für die Abnahme des Magnetowiderstandes mitverantwortlich ist.

Zusätzlich wirkt die $\mathrm{Ni}$-Benetzung der $\mathrm{Cu}$-Korngrenze ab einer gewissen Konzentration vermutlich als ferromagnetische Brücke, die eine GMR-Amplituden Abnahme noch verstärkt.

Durch Präparation planarer Proben für die Analyse in der Tomographischen Atomsonde konnte der Nachweis erbracht werden, daß die Ergebnisse von gesputterten Schichten auf W-Trägerspitzen direkt vergleichbar sind mit Reaktionsmechanismen in planar gewachsenen Schichten.

Nur durch die Detailkenntnis der Mikrostruktur und ihre Bedeutung für die Reaktionsmechanismen wird eine weitere Entwicklung und Verbesserung von GMR-Sensoren, gerade im Hinblick auf zukünftige Miniaturisierung, für die industrielle Anwendung möglich sein. 


\section{Abschlussbemerkung}

In den vorangegangen Kapiteln wurde an den Systemen $\mathrm{Ag} / \mathrm{Al}$ und $\mathrm{Co} / \mathrm{Cu} / \mathrm{Py}$ erfolgreich der Einfluß der Mikrostruktur auf den Reaktionsprozeß deutlich gemacht. Die Untersuchungen mit der Tomographischen Atomsonde haben gezeigt, daß sich die Reaktionsmechanismen wesentlich komplexer darstellen, als die häufig in Modellen angenommene Lagengeometrie.

Das vermeintlich einfache Modellsystem Ag/Al offenbart in der Nanoanalyse eine komplexe 3D-Reaktionsmorphologie. Die Keimbildung der erwarteten Phase findet nicht an der Grenzfläche statt, sondern bevorzugt in den Tripelpunkten der Ag-Korngrenzen. In weiteren Reaktionsschritten kommt es zur Ausbildung einer ausgedehnten intermediären Phase mit der Struktur der Gleichgewichtsphase, aber abweichend von ihrer Konzentration. Die Einstellung der Gleichgewichtskonzentration erfolgt merklich erst bei höherer Temperatur durch Volumendiffusion.

Das Schichtsystem $\mathrm{Co} / \mathrm{Cu} / \mathrm{Py}$ ist ein Beispiel dafür, daß selbst nach Vorliegen einer präparierten Lagenstruktur die Reaktionsmechanismen keineswegs vorgegeben sind. Anhand der Nanoanalyse konnte die klare Abhängigkeit des Reaktionsverlaufes von der Mikrostruktur, insbesondere den Gitterfehlern aufgezeigt werden. Als charakteristisch wurde eine starke $\mathrm{Fe}-$ und $\mathrm{Ni}$-Segregation durch die Korngrenzen der $\mathrm{Cu}-\mathrm{Zwischenschicht} \mathrm{offengelegt.} \mathrm{Es} \mathrm{liegt} \mathrm{nahe,} \mathrm{die} \mathrm{Fe-Verarmung} \mathrm{der} \mathrm{Py-Lage}$ und anschließende Legierungsbildung innerhalb der Co-Schicht für die Abnahme des GMR-Effektes verantwortlich zu machen. Die Ausbildung einer ferromagnetischen Brücke durch eine starke $\mathrm{Ni}$-Benetzung der $\mathrm{Cu}-$ Korngrenze wirkt vermutlich noch verstärkend auf die Störung der GMR-Amplitude.

Neben der direkten Beschichtung von spitzenförmigen Proben für die TAP-Analyse wurde ein eigens entwickeltes lithographisches Verfahren zur Spitzenpräparation benutzt. Damit gelang es, aus planaren Schichtsystemen Proben in Spitzengeometrie mit einem Krümmungsradius kleiner $50 \mathrm{~nm}$ zur Analyse zu präparieren. Hierdurch sind die möglicherweise herstellungsbedingt unterschiedlichen Mikrostrukturen und ihr Einfluß auf den Reaktionsablauf direkt vergleichbar. Im Fall des $\mathrm{Co} / \mathrm{Cu} / \mathrm{Py}-\mathrm{Schich}$ tsystems zeigte sich in der lithographisch präparierten Probe die gleiche starke Fe-Segregation, wie bei dem auf die Trägerspitze aufgebrachtem Schichtpaket. Beide Herstellungsmethoden führen bezüglich des Reaktionsverlaufes also zu den gleichen Charakteristika.

Nur durch die Detailkenntnis der Mikrostruktur ist eine Anpassung der Modelle an die reale Reaktionsmorphologie möglich. Zudem ist die atomare chemische Charakterisierung für eine zielgerichtete Optimierung der Systeme im Hinblick auf den Anwendungsbereich unerläßlich. 


\section{Anhang}

\section{TRIM-Simulationen zu Al/Ag-Schichten}

Wie in Abschnitt 4.3.2 dargestellt, zeigt das as-sputtered Profil des rekonstruierten Al/Ag-Schichtpaketes bereits eine Breite des Interfaces von $1 \mathrm{~nm}$. Nun ist der Frage nachzugehen, inwieweit der Sputterprozeß, insbesondere der Effekt des Ionenmischens beim Beschichten, einen Einfluß auf die Grenzflächenschärfe hat. Ebenfalls ist bei der Untersuchung der bei $90^{\circ} \mathrm{C}$ für 11 Minuten ausgelagerten Probe die Frage nach einer Leerstellenübersättigung bedingt durch den Herstellungsprozeß aufgekommen. Diese hohe Konzentration an Leerstellen hätte einen beschleunigenden Einfluß auf die Interdiffusion der Schichten.

Zur Beantwortung dieser Fragen wurden Monte-Carlo-Simulationen mit dem Programm SRIM-2000, Version 39, auf konventionellen PC's durchgeführt. Eine genaue Beschreibung der Software findet sich in [ZIEGLER, IBM]. Simuliert wird die Wechselwirkung eines auf einen amorphen Festkörper treffendes Teilchen bestimmter Energie unter zufälliger Wahl des Stoßwinkels. Bei solch einem Stoßprozeß wird kinetische Energie übertragen und entsprechend, falls ein Atom seinen Platz verläßt, die Bindungsenergie $E_{1}$ (Lattice Binding Energy) abgezogen. Beide Teilchen können mit ihrer Energie weitere Stoßprozesse initiieren, bis hin zur Entstehung ganzer Stoßkaskaden. Fällt die Energie eines Teilchens unter einen festgesetzten Schwellwert $E_{d}$ (Displacement Energy), finden keine weiteren Stoßprozesse mehr statt. Gelangen unter diesem Prozeß Teilchen zur Oberfläche wird anhand einer festgelegten Bindungsenergie $E_{s}$ (Surface Binding Energy) entschieden, ob sie genügend Energie besitzen, um aus der Oberfläche auszutreten. Über die Summation all dieser auftretenden Effekte gelangt man dann zu Aussagen bezüglich der Eindringtiefe, Anteil der rückgestreuten Ionen und auch dem abgesputtertem Material. Hervorzuheben ist aber, daß diese Rechnungen nicht den Anspruch haben, quantitativer Richtigkeit zu genügen, sondern lediglich qualitative Möglichkeiten aufzeigen sollen. Dabei wurden Schichten üblicher Dicke von $30 \mathrm{~nm}$ mit den in Tabelle 7 angegebenen Parametern simuliert.

\begin{tabular}{|l|l|l|}
\hline & Al & Ag \\
\hline Masse [amu] & 26,982 & 107,87 \\
\hline Dichte [g/cm $\left.{ }^{3}\right]$ & 2,702 & 10,473 \\
\hline $\begin{array}{l}\mathbf{E}_{1}- \\
\text { Lattice Binding Energy [eV] }\end{array}$ & 3 & 3 \\
\hline $\begin{array}{l}\mathbf{E}_{\mathrm{s}}- \\
\text { Surface Binding Energy [eV] }\end{array}$ & 3,36 & 2,97 \\
\hline $\begin{array}{l}\mathbf{E}_{\mathrm{d}}- \\
\text { Displacement Energy [eV] }\end{array}$ & 25 & 25 \\
\hline
\end{tabular}

Tabelle 7: Parameter der Simulation 
Die Energien der beim primären Sputterprozeß ( $\mathrm{Ar} \rightarrow$ Target) erzeugten $\mathrm{Al}-/ \mathrm{Ag}-$ Atome sind in Tabelle 8 zu finden. Wie erwartet liegen die Energien der austretenden Al-Atome mit 13,7 eV niedriger als die Energie der Ag-Atome. Es ist jedoch zu beachten, daß bei allen Proben immer zuerst die Ag-Lage auf die W-Trägerspitze deponiert wurde. Für den Beschuß dieser Ag-Lage mit Al-Teilchen von $14 \mathrm{eV}$, liefert die Simulation im Ergebnis eine Durchmischung in der Tiefe von $3 \AA$. Ebenfalls wird eine signifikante Leerstellenbildung nicht angezeigt.

\begin{tabular}{|l|c|c|c|}
\hline Primär-Sputterprozeß & Winkel & Eindringtiefe & max. Energie extr. Teilchen \\
\hline $\mathrm{Ar}(600 \mathrm{eV}) \rightarrow \mathrm{Ag}$ & $45^{\circ}$ & $11 \AA$ & $20,9 \mathrm{eV} \mathrm{Ag}-$ Atom \\
\hline $\mathrm{Ar}(600 \mathrm{eV}) \rightarrow \mathrm{Al}$ & $45^{\circ}$ & $16 \AA$ & $13,7 \mathrm{eV} \mathrm{Al}-$ Atom \\
\hline Sekundär-Prozeß & \multicolumn{3}{|l}{} \\
\hline $\mathrm{Ag}(21 \mathrm{eV}) \rightarrow \mathrm{Al}$ & $0^{\circ}$ & $7 \AA$ & $\begin{array}{c}\text { keine Resputtereffekte / keine } \\
\text { Leerstellenbildung }\end{array}$ \\
\hline $\mathrm{Al}(14 \mathrm{eV}) \rightarrow \mathrm{Ag}$ & $0^{\circ}$ & $3 \AA$ & $\begin{array}{c}\text { keine Resputtereffekte / keine } \\
\text { Leerstellenbildung }\end{array}$ \\
\hline
\end{tabular}

Tabelle 8: Ergebnisse der SRIM-Simulation

Mit diesen berechneten Eindringtiefen läßt sich die gemessene Durchmischungszone von $1 \mathrm{~nm}$ nicht erklären. Hier müssen andere Effekte noch von Bedeutung sein. 


\section{Abkürzungsverzeichnis}

DSC Dynamische Differentialkalorimetrie (Dynamic scanning calorimetry)

EMS Elektronenstrahlmikrosonde

FIM Feldionenmikroskop

GMR Giant-Magneto-Resistance (Riesenmagnetowiderstand)

MCP Mikrokanalplatte

RBS Rutherford-Backscattering

SIMS Sekundär-Ionen-Massen-Spektrometer

TAP Tomographische Atomsonde

TEM Transmissionselektronenmikroskop

TRIM Transport of Ions in Matter

UHV Ultrahochvakuum

XRD Röntgendiffraktometrie 


\section{Literaturverzeichnis}

[ADDA] Adda, Y., Philibert, J.,

Diffusion dans les solides, tome I. et II., Presses Universitaires de France, Paris 1966

[ALEXANDER] Alexander, W.B., Slifkin, L.M.,

Phys.Rev.B 1 (1970) 3274

[AL-KASSAB] Al-Kassab, T., Wollenberger, H., Schmitz, G. und Kirchheim, R.,

High Imaging and Spectroscopy of Materials, Kapitel „,Tomography by Atom

Probe“, Springer Verlag, Berlin, Hrsg. M. Rühle und F. Ernst, to be published

[Aubertin] Aubertin, F., Gonser, U., Campbell, St., Wagner, H.-G.,

Z.Metallkunde, 76 (1985) 237

[AVRAMI] Avrami, M.,

J.Chem.Phys. 7 (1939) 1103

[BAGLIN] Baglin, J.E.E., d'Heurle, F.M., Hammer, W.N.,

J.Appl.Phys., 50 (1978) 266

[BAIBICH] Baibich, M.N., Broto, J.M., Fert, A., Nguyen van Dau, F., Petroff, F., Eitenne, P., Creuzet, G., Friedrich, A., Chazelas, J.,

Phys.Rev.Lett. 61 (1988) 2472

[BARMAK] Barmak, K., Michaelsen, C., Lucadamo, G.,

J.Mater.Res. 12 (1997) 133

[BAUR1] Baur, R. und Gerold, V., Acta Met. 10 (1962) 637

[BAUR2] Baur, R. und Gerold, V., Z. Metallkunde 52 (1961) 671

[BENÉ] Bené, R.,

J.Appl.Phys. 61 (1987) 1826

[Berkowitz] Berkowitz, A.E., Mitchell, J.R., Carey, M.J., Young, A.P., Zhang, S., Spada, F.E., Parker, F.T., Hütten, A.,

Phys.Rev.Lett. 68 (1992) 3745

[BINASCH] Binasch, G., Grünberg, P., Saurenbach, F., Zinn, W., Phys.Rev.B 39 (1989) 4828

[Blavette1] Blavette, D., Duval, P., Letellier, L. und Guttmann, M., Acta mater. 44 (1996) 4995

[Blavette2] Blavette, D., Deconihout, B., Bostel, A., Sarreau, J., Bouet, M. und Menand, A., Rev. Sci. Instrum. 64 (1993) 2911

[Вово] Bobo, J.F., Hennet, L., Piecuch, M., Europhys.Lett. 24 (1993) 139 
[BORMANN] Bormann, R.,

Mat.Res.Soc.Symp.Proc. Vol.343 (1994)

[Bostel] Bostel, A., Blavette, D., Menand, A. und Sarreau J., J. de Phys. C8 50 (1989) 501

[Bronstein] Bronstein, I.N., Semendjajew, K.A., Musiol, G., Mühlig, H.,

Taschenbuch der Mathematik, Verlag Harri Deutsch, Thun, Frankfurt a.M., 1995

[Bruno] Bruno, P., Chappert, C.,

Phys.Rev.Lett. 67 (1991) 1602

[CAHN] Cahn, R.W. und Haasen, P., Ed.,

Physical Metallurgy, Elsevier Science B.V., North-Holland, 1996

[CAmPISAnO] Campisano, S.U., Foti, G., Rimini, E., Lau, S.S., Mayer, J.W., Philos.Mag. 31 (1975) 903

[CARBOnE] Carbone, C., Alvarado, S.F., Phys.Rev.B 36 (1987) 2433

[COFFEY] Coffey, K.R., Clevenger, L.A., Barmak, K., Rudman, D.A., Thompson, C.V., Appl.Phys.Lett. 55 (1989) 852

[CULL] Cullity, B.D., Elements of X-Ray Diffraction, Addison-Wesley Publishing Company, Inc., Reading, 1967

[DEAL] Deal, B.E., Grove, A.S., J.Appl.Phys. 36 (1965) 3770

[DIENY] Dieny, B., Speriosu, V.S., Gurney, B.A., Parkin, S.S.P., Wilhoit, D.R:, Roche, K.P., Metin, S., Peterson, D.T., Nadimi, S., J.Magn.Magn.Mater. 93 (1991) 101

[DRECHSLER] Drechsler, M., Wolf, P.,

Zur Analyse von Feldionenmikroskop - Aufnahmen mit atomarer Auflösung, Vierter Internationaler Kongreß für Elektronenmikroskopie-Verhandlungen, Springer Verlag, Berlin, 1960

[EWERT] Ewert, J.C., Hartung, F., Schmitz, G., Appl. Phys. Lett. 71 (10) 1997

[FoGELSON] Fogelson, R.L., Ugay, Ya.A., Akimova, I.A., Izv.Vyssh.Uchebn.Zaved., Tsvetn.Metall. (2) 1975, 142

[GÄRTNER] Gärtner, F.,

Dissertation, Georg-August-Universität Göttingen, 1992

[GEBER] Geber, G., Al-Kassab, T., Isheim, D., Busch, R. und Haasen, P., Z. Metallkd., 83 (6), 449-456 (1992)

[GösELE1] Gösele, U., Tu, K.N., J.Appl.Phys. 66 (1989) 2619 
[GÖSELE2] Gösele, U., Tu, K.N., J.Appl.Phys. 53 (1982) 3252

[GRÜNBERG] Grünberg, P., Schreiber, R., Pang, Y., Brodsky, M.B., Sowers, H., Phys.Rev.Lett. 57 (1986) 2442

[HAASEN] Haasen, P.,

Physikalische Metallkunde, Springer-Verlag, 1994

[HALl] Hall, P.M., Morabito, J.M., Panousis, N.T., Thin solid films, 41 (1977) 341

[HAMP] Hamp, M.,

Diplomarbeit, Georg-August-Universität Göttingen, 1997

[HEINRICH] Heinrich, B., Bland, J.A.C.,

Ultrathin Magnetic Structures II, Springer Verlag, Berlin, 1994

[HEIT1] Heitmann, S.

Diplomarbeit, Universität Bielefeld, 2000

[HeIT2] Heitmann, S., Hütten, A., Hempel, T., Schepper, W., Reiss, G., Alof, C., J.Appl.Phys. 87 (2000) 4849

[Heurle1] d’Heurle, F.M., Gas, P., Philibert, J., Def.Diff.Forum 143 (1997) 529

[HEURLE2] d'Heurle, F.M., Gas, P., J.Mater.Res. 1 (1986) 205

[HILlERT] Hillert, M., Averbach, B.C., Cohen, M., Acta Metall. 4 (1956) 31

[Hono] Hono, K., Hasegawa, N., Okano, R., Fujimori, H., Sakurai, T., Appl.Surf.Sci., 67 (1-4) 407, 1993

[Hoscoe] Hoscoe, Y., Hoshino, K., Tsunashima, S., Uchiyama, S., Imura, R., IEEE Trans.Magn. 28 (1992) 2665

[HÜTTEN] Hütten, A., persönliche Mitteilung

[Hwang] Hwang, D.G., Lee, S.S., Lee, K.A., Park, C.M., Kim, M.Y., Choi, K.R., Choi, S.J., Lee, Y.H., Rhee, J.R.,

IEEE Trans.Magn. 32 (1996) 4579

[IBM] IBM Corp., http://www.research.ibm.com/ionbeams/SRIM/SRIMA.HTM, 1996

[JESKE1] Jeske, T., Schmitz, G.,

Mat.Sci.Eng. A (2000) in press

[JESKE2] Jeske, T., Schmitz, G., Kirchheim, T., Mat.Sci.Eng. A 270 (1999) 64 
[Johnson1] Johnson, M.T., Purcell, S.T., McGee, N.W.E., Cohehoorn, R., aan de Stegge, J., Hoving, W.,

Phys.Rev.Lett. 68 (1992) 2688

[JOHNSON2] Johnson, W.L.,

Amorphization by interfacial reactions, Materials Interfaces, Wolf \& Yip, 1992

[JoHNSON3] Johnson, W.A., Mehl, R.F.,

Trans.AIME 135 (1939) 416

[KITTEL] Kittel, C.,

Einführung in die Festkörperphysik, R. Oldenbourg Verlag, München 1996

[KREBS1] Krebs, H.U.,

Dissertation, Georg-August-Universität Göttingen, 1984

[KREBS2] Krebs, H.U.,

J.Non-Crystalline Solids 61-62 (1984) 463

[LAM] Lam, N.Q., Rothman, S.J., Mehrer, H., Nowicki, L.J.,

Phys.Status Solidi (b) 57 (1973) 225

[LANDOLT] Landolt-Börnstein, Mehrer, H. Hrsg, Band 26, Berlin 1990

[LANG] Lang, C.,

Diplomarbeit, Georg-August-Universität Göttingen, 1998

[LARSON1] Larson, D.J., Petford-Long, A.K., Cerezo, A.,

Mat.Sci.Eng. A 270 (1999) 69

[LARSON2] Larson, D.J., Foord, D.T., Petford-Long, A.K., Liew, H., Blamire, M.G., Cerezo, A., Smith, G.D.W.,

Ultramicroscopy 79 (1999) 287

[LIM] Lim, S.S., Rossiter, P.L., Tibballs, J.E.,

Calphad, 19 (1995) 131

[LotTIS] Lottis, D., Fert, A., Morel, R., Pereira, L.,

J.Appl.Phys. 73 (1993) 5515

[LoYen] v.Loyen, L., Elefant, D., Tietjen, D., Schneider, C.M., Hecker, M., Thomas, J., J.Appl.Phys. 87 (2000) 4852

[LuO] Luo, Y., Moske, M., Käufler, A., Lorenz, T., Samwer, K., Europhys.Lett. 37 (1997) 225

[MAJNI] Majni, G., Nobili, C., Ottaviani, G., Costato, M., Galli, E., J.Appl.Phys. 52 (1981) 4047

[MAO] Mao, M, Cerjan, C., Gibbons, M., Law, B., Grabner, F., Vernon, S.P., Wall, M., IEEE Trans.Mag. 34 (1998) 915

[MARKWITZ] Markwitz, A., Matz, W., Waldschmidt, M., Demortier, G.,

Surf. Interface Anal. 26 (1998) 160 
[MASSALSKI] Massalski, T.B.,

Binary Alloy Phase Diagrams, ASM International 1990

[MENG] Meng, S.,

Mat.Sci.Eng. 97 (1988) 87

[MerTiG] Mertig, I.,

Magnetische Schichsysteme, Ferienkurs Jülich 1999

[MichaELSEN1] Michaelsen, C., Lucadamo, G., Barmak, K.,

J.Appl.Phys., 80 (1996) 6689

[MichaELSEN2] Michaelsen, C., Lucadamo, G., Barmak, K., J.Appl.Phys., 80 (1996) 6689

[MILLER] Miller, M.K., Cerezo, A., Hetherington, M. und Smith, G.D.W., Atom Probe Field Ion Microscopy, Clarendon Press, Oxford, 1996

[MROZEK] Mrozek, S.,

Diplomarbeit, Universität Bielefeld, 1998

[MÜLLER] Müller, E.W., Tsong, T.T.,

Field Ion Microscopy, Elsevier Publishing Company, New York, 1969

[NICHOLSEN] Nicholsen, R.B., Nutting, J.,

Acta Metall. 9 (1961) 332

[OSAmura] Osamura, K., Nakamura, T., Kobayashi, A., Hashizume, T. und Sakurai, T., Skripta Met., 21 (1987) 255

[PARKIN] Parkin, S.S.P., Rabedeau, T., Appl.Phys.Lett. 68 (1996) 1162

[Pretorius1] Pretorius, R., Vredenberg, A.M., Saris, F.W., de Reus, R., J.Appl.Phys. 70 (1991) 3636

[PRETORIUS2] Pretorius, R., de Reus, R., Vredenberg, A.M., Saris, F.W., Mater.Lett. 9 (1990) 494

[PUNDT] Pundt, A.,

Dissertation, Georg - August - Universität Göttingen, 1995

[RÄTZKE] Rätzke, K., Hall, M.J., Jardine, D.B., Shih, W.C., Somekh, R.E., Greer, A.L., J.Magn.Magn.Mater. 204 (1999) 61

[RoY] Roy, R., Sen, S.K., J.Mat.Sci., 27 (1992) 6098

[SCHLEIWIES1] Schleiwies, J.,

Diplomarbeit, Georg - August - Universität Göttingen, 1998

[SCHLEIWIES2] Schleiwies, J., Schmitz, G.,

Mat.Sci.Eng. A (im Druck) 
[SCHLEIWIES3] Schleiwies, J., Schmitz, G., Heitmann, S., Hütten, A., Appl.Phys.Lett. (eingereicht)

[SCHLIEBE] Schliebe, T.,

Dissertation, Georg - August - Universität Göttingen, 1997

[Schmitz1] Schmitz, G., Svenson, O., Troche, P. und Harbsmeier, F.,

Proc.Solid-Solid Phase Transformations, The Japan Institute of Metalls, (1999) 1283

[SCHMITZ2] Schmitz, G.,

Dissertation, Georg - August - Universität Göttingen, 1994

[SVENSON] Svenson, O.,

Diplomarbeit, Georg-August-Universität Göttingen, 1999

[ThuVANDER] Thuvander, M, Andrén, H.-O., Stiller, K., Hu, Q.-H.,

Ultramicroscopy 73 (1998) 279

[TROCHE] Troche, P.,

Dissertation, Georg - August - Universität Göttingen, 2000

[Ungár] Ungár, T., Dubey, Ph.A., Kostorz, G., Acta Metall.mater., 38 (1990) 2583

[VoLIN] Volin, T.E., Balluffi, R.W., Phys.Status Solidi 25 (1968) 163

[WALSER] Walser, R.M., Bené, R.W., Appl.Phys.Lett., 28 (1976) 624

[WEAVER] Weaver, C., Brown, L.C., Phil.Mag., 17 (1968) 671

[WESTMOR] Westmoreland, J.E., Weisenberger, W.H., Thin Solid Films 19 (1973) 349

[WITTIG] Wittig, F.E., Schilling, W., Z.Metallk., 50 (1959) 610

[ZAHN1] Zahn, P., Binder, J., Mertig, I., Zeller, R., Dederichs, P.H., Phys.Rev.Lett. 80 (1998) 4309

[ZAHN2] Zahn, P., Mertig, I., Richter, M., Eschrig, H., Phys.Rev.Lett., 75 (1995) 2996

[ZIEGLER] Ziegler, J.F., Biersack, J.P. und Littmark, U.,

The stopping and Range of Ions in Solids, Pergamon Press, New York, 1985 


\section{Danksagung}

An dieser Stelle möchte ich allen ausdrücklich danken, die zum Gelingen meiner Arbeit beigetragen haben.

Herrn Prof. Dr. R. Kirchheim danke ich, daß er mir ermöglicht hat meine Arbeit nach dem Diplom am Institut für Materialphysik fortzusetzen. Sein Interesse am Fortgang meiner Arbeit und seine ständige Gesprächsbereitschaft waren für mich immer sehr motivierend.

Einen besonderen Dank möchte ich auch an Dr. Guido Schmitz richten, für die vielen intensiven, aber auch anstrengenden Gespräche. Sie haben meine Neugier auf immer neue Aspekte der Physik noch verstärkt. - Auch während seines USA-Aufenthaltes hat er die stets gute Betreuung ohne Unterbrechung fortgesetzt, durch Nutzung aller möglichen Hilfsmittel.

Mein Zimmerkollege Christian Lang hat durch seine außerordentliche Teamarbeit und Hilfsbereitschaft immer für eine produktive und freundschaftliche Arbeitsatmosphäre gesorgt.

Den weiteren Mitgliedern der FIM (TAP)-Gruppe, Tobias Jeske, Christian Kluthe, Pyuck-Pa Choi, Alexander Heinrich, Daniel Wolde-Giorgis und Dr. Talaát Al-Kassab danke ich für die gute Zusammenarbeit, auch wenn das Gerangel um Meßtermine doch manches mal an einen Türkischen Basar erinnerte.

Herrn Dr. A. Hütten und S. Heitmann möchte ich für die gute Teamarbeit, für die umfangreiche Bereitstellung von Proben und für die unermüdliche Beantwortung noch so vieler Fragen zu magnetischen Eigenschaften danken.

J. Bankmann und Dr. P. Wilbrandt haben mich bei den SIMS-Messungen und dem "Anschnitzen" der zahlreichen TAP-Spitzen sehr unterstützt.

Dem Institut für Röntgenphysik in Göttingen, insbesondere Herrn Dr. M. Peuker danke ich für die immer prompte Strukturierung der Schichten mit dem "Löwen".

Der Deutschen Forschungsgemeinschaft möchte ich für die finanzielle Unterstützung der Arbeit danken.

Natürlich sind auch die fleißigen Helfer in den Werkstätten des Institutes nicht vergessen. Ihnen sei für die reibungslose und unbürokratische Hilfe in so manchen Situationen gedankt.

Last but not least...

Meiner Frau, Susanne Carmen danke für ihre großartige Unterstützung sowohl in den Hoch- als auch in den Tiefphasen der Arbeit, wenn mal wieder alle Spitzen abgerissen waren. Ohne ihre Hilfe wäre diese Arbeit nicht zu diesem Abschluß gekommen. 


\section{Lebenslauf}

\begin{tabular}{|c|c|}
\hline & \begin{tabular}{|ll} 
Persönliche Daten & \\
Familienstand: & Verheiratet \\
Nationalität: & Deutsch \\
Geburtstag / -Ort: & $07.12 .1971 \quad$ Lingen \\
Eltern: & Inge \& Hans-Jürgen Schleiwies
\end{tabular} \\
\hline $07 / 1984-06 / 1991$ & $\begin{array}{l}\text { Schulbildung } \\
\text { Otto-Hahn-Gymnasium, Göttingen } \\
\text { Allgemeine Hochschulreife }\end{array}$ \\
\hline $07 / 1991-09 / 1992$ & $\begin{array}{l}\text { Wehrdienst } \\
\text { Grundwehrdienst in Göttingen }\end{array}$ \\
\hline $10 / 1992-07 / 1998$ & $\begin{array}{l}\text { Studium } \\
\text { Diplom-Physik Studium an der Georg-August-Universität, } \\
\text { Göttingen }\end{array}$ \\
\hline $10 / 1996-07 / 1998$ & $\begin{array}{l}\text { Diplomarbeit am Institut für Materialphysik über } \\
\text { "Mikrocharakterisierung von nanostrukturierten } \mathrm{Cu} / \mathrm{Nb}- \\
\text { Multilagen" }\end{array}$ \\
\hline 07/1998 & Diplomprüfung im Fach Physik \\
\hline seit $08 / 1998$ & $\begin{array}{l}\text { Wissenschaftlicher Mitarbeiter am Institut für } \\
\begin{array}{l}\text { Materialphysik an der Georg-August-Universität, } \\
\text { Göttingen }\end{array}\end{array}$ \\
\hline
\end{tabular}

